\title{
Contingency Interim Measure for the Public Water Supply at Barnes, Kansas
}

\section{Environmental Science Division}


About Argonne National Laboratory

Argonne is a U.S. Department of Energy laboratory managed by UChicago Argonne, LLC under contract DE-AC02-06CH11357. The Laboratory's main facility is outside Chicago, at 9700 South Cass Avenue, Argonne, Illinois 60439. For information about Argonne and its pioneering science and technology programs, see www.anl.gov.

\section{Availability of This Report}

This report is available, at no cost, at http://www.osti.gov/bridge. It is also available on paper to the U.S. Department of Energy and its contractors, for a processing fee, from:

U.S. Department of Energy

Office of Scientific and Technical Information

P.O. Box 62

Oak Ridge, TN 37831-0062

phone (865) 576-8401

fax (865) 576-5728

reports@adonis.osti.gov

\section{Disclaimer}

This report was prepared as an account of work sponsored by an agency of the United States Government. Reference herein to any specific commercial product, process, or service by trade name, trademark, manufacturer, or otherwise, does not necessarily constitute or imply its endorsement, recommendation, or favoring by the United States Government or any agency thereof. The views and opinions of document authors expressed herein do not necessarily state or reflect those of the United States Government or any agency thereof, Argonne National Laboratory, or UChicago Argonne, LLC. 


\section{Contingency Interim Measure for the Public Water Supply at Barnes, Kansas}

by

Applied Geosciences and Environmental Management Section

Environmental Science Division, Argonne National Laboratory

June 2009

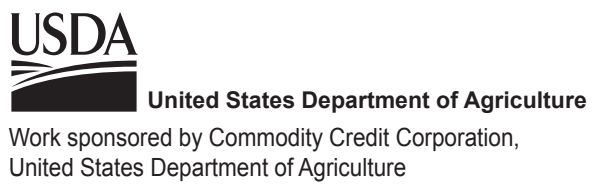




\section{Contents}

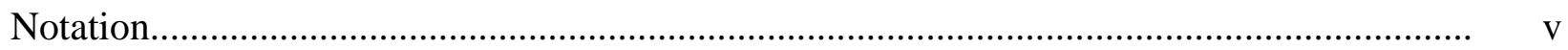

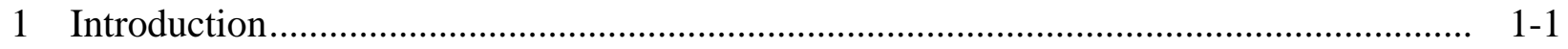

2 Site Background............................................................................................................ 2-1

3 Summary of Results................................................................................................. $3-1$

3.1 Previous Investigations of Carbon Tetrachloride Contamination................................ 3-1

3.2 Geologic and Hydrogeologic Conditions...................................................................... 3-3

4 Proposed Interim Measure ........................................................................................... 4-1

4.1 Interim Measure Evaluation................................................................................. 4-1

4.1.2 Option 1 - Replacement of Current Water Supply ...................................... 4 4-1

4.1.2 Options 2 and 3 - GAC and Air Stripping................................................... 4-2

4.2 Interim Measure Implementation.............................................................................. 4-3

5 Interim Treatment System Objectives ……………........................................................... 5-1

6 Interim Measure Design.................................................................................................. 6-1

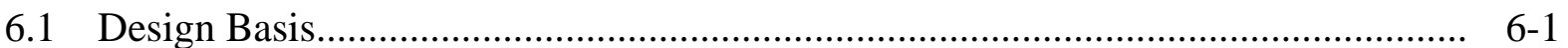

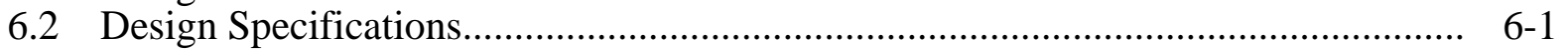

6.3 Drawings/Schematics.......................................................................................... 6-3

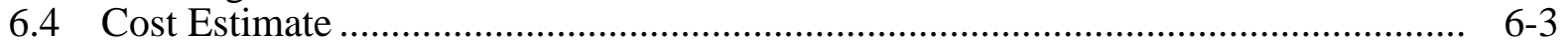

6.5 Proposed Working Schedule.................................................................................. 6-4

6.6 Potential Implementation Issues ........................................................................... 6-4

7 Status of Treatment System Design Appendices .................................................................... 7-1

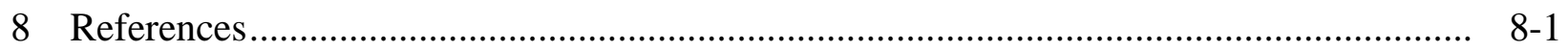

Appendix A: Technical Analysis of the Barnes School as a Potentially Responsible Party.. A-1

Appendix B: Historical Analytical Results and Well Completion Information for the Barnes Public Water Supply................................................................ B-1

\section{Tables}

2.1 Analytical results for carbon tetrachloride and chloroform in the public supply wells at Barnes in 1986-2008

3.1 Analytical results for volatile organic compounds in groundwater samples collected at Barnes in July 2006 to July 2008.

3.2 Hand-measured water levels at Barnes in November 2007 to July 2008 ....... 


\section{Tables (Cont.)}

6.1 Public water supply systems in Kansas where granular activated carbon vessels have been or are currently being used to treat groundwater contaminated with volatile organic compounds

6.2 Working schedule for treatment system installation

B.1 Historical analytical data for wells at Barnes in 1986-2005.

B.2 Construction summary for wells at Barnes

\section{Figures}

2.1 Location of Washington County and Barnes, Kansas ............................................... 2-5

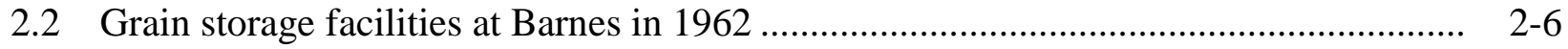

2.3 Location of the former CCC/USDA facility, other relevant structures and facilities, and the public water supply wells at Barnes

3.1 Carbon tetrachloride concentrations detected in groundwater in the KDHE's 1999 comprehensive investigation and subsequent sampling....

3.2 Analytical results for carbon tetrachloride in groundwater samples collected during the 2006-2007 CCC/USDA-Argonne investigation at Barnes

3.3 Analytical results for chloroform in groundwater samples collected during the 2006-2007 CCC/USDA-Argonne investigation at Barnes

3.4 Analytical results for carbon tetrachloride in groundwater samples collected during CCC/USDA-Argonne monitoring in November 2007-July 2008 at Barnes

3.5 Analytical results for chloroform in groundwater samples collected during CCC/USDA-Argonne monitoring in November 2007-July 2008 at Barnes.

3.6 Locations of interpretive hydrogeologic cross sections

3.7 West-east interpretive hydrogeologic cross section A-A' across the former $\mathrm{CCC/USDA}$ grain storage property at Barnes, illustrating the lateral and vertical distribution of carbon tetrachloride in soil and groundwater in 2006-2007

3.8 West-east interpretive hydrogeologic cross section B-B' at Barnes, illustrating the lateral and vertical distribution of carbon tetrachloride in soil and groundwater north of the former CCC/USDA property in 2006-2007

3.9 West-east interpretive hydrogeologic cross section C-C' across the former $\mathrm{CCC} / \mathrm{USDA}$ grain storage property at Barnes, illustrating the lateral and vertical distribution of carbon tetrachloride in soil and groundwater in 2006-2007. 


\section{Figures (Cont.)}

3.10 North-south interpretive hydrogeologic cross section D-D' across the former CCC/USDA grain storage property at Barnes, illustrating the lateral and vertical distribution of carbon tetrachloride in soil and groundwater in 2006-2007

3.11 Interpreted carbon tetrachloride plumes in wells screened in the intermediate aquifer in March and July 2008

3.12 Interpreted carbon tetrachloride plumes in wells screened in the deep aquifer in March and July 2008

3.13 Hydrographs summarizing monthly results of long-term water level monitoring in wells at Barnes in January-June 2008.

3.14 Potentiometric surface maps depicting the groundwater flow direction at Barnes in January-April 2007, before pumping of the public water supply wells and during pumping

3.15 Potentiometric surface maps depicting the groundwater flow direction at Barnes in December 2007 and May 2008, before pumping of the public water supply wells and during pumping....

A.1 Carbon tetrachloride concentrations in soil gas samples collected by the KDHE in 1996 to investigate two source areas at Barnes

A.2 The school buildings at Barnes, July 2008. 


\section{Notation}

\begin{tabular}{ll} 
AMSL & above mean sea level \\
BER & Bureau of Environmental Remediation \\
BGL & below ground level \\
BTEX & benzene, toluene, ethylbenzene, xylene \\
CCC & Commodity Credit Corporation \\
EPA & U.S. Environmental Protection Agency \\
ft & foot (feet) \\
GAC & granular activated carbon \\
gal & gallon(s) \\
gpm & gallon(s) per minute \\
IM & interim measure \\
in. & inch(es) \\
KDHE & Kansas Department of Health and Environment \\
$\mu$ g/kg & microgram(s) per kilogram \\
$\mu$ g/L & microgram(s) per liter \\
MCL & maximum contaminant level \\
mi & mile(s) \\
ppb & part(s) per billion \\
ppm & part(s) per million \\
PWS & public water supply \\
RBSL & risk-based screening level \\
RWD & Rural Water District \\
TOC & top of casing \\
USD 223 & Unified School District 223 \\
USDA & U.S. Department of Agriculture \\
VOC & volatile organic compound \\
\hline &
\end{tabular}




\section{Contingency Interim Measure for the Public Water Supply at Barnes, Kansas}

\section{Introduction}

This document presents a conceptual design for a contingency interim measure (IM) for treatment of the public water supply system at Barnes, Kansas, should this become necessary.

The aquifer that serves the public water supply system at Barnes has been affected by trace to low concentrations of carbon tetrachloride and its degradation product, chloroform. Investigations conducted on behalf of the CCC/USDA by Argonne National Laboratory (Argonne 2008a) have demonstrated that groundwater at the Barnes site is contaminated with carbon tetrachloride at concentrations exceeding the Kansas Tier 2 risk-based screening level (RBSL) and the EPA maximum contaminant level (MCL) of $5.0 \mu \mathrm{g} / \mathrm{L}$ for this compound. The Commodity Credit Corporation of the U.S. Department of Agriculture (CCC/USDA) formerly operated a grain storage facility in Barnes, approximately $800 \mathrm{ft}$ east-southeast of the public water supply wells. Carbon tetrachloride was used in the treatment of grain.

Another potential source identified in an investigation conducted for the KDHE (PRC 1996) is the site of a former agriculture building owned by the local school district (USD 223). This building is located immediately east of well PWS3.

The potential contingency IM options evaluated in this report include the treatment of groundwater at the public water supply wellheads and the provision of an alternate water supply via Washington County Rural Water District \#2 (RWD 2).

This document was developed in accordance with KDHE Bureau of Environmental Remediation (BER) Policy \#BER-RS-029 (Revised) (KDHE 2006a), supplemented by guidance from the KDHE project manager. Upon the approval of this contingency IM conceptual design by the KDHE, the CCC/USDA will prepare a treatment system design document that will contain the following elements:

- Description of the approved contingency IM treatment method 
- Drawings and/or schematics provided by the contractor and/or manufacturer of the approved technology

- A detailed work schedule with a generic timetable

- A detailed operations and maintenance plan

- A proposed performance monitoring plan

- A fact sheet associated with a community relations plan 


\section{Site Background}

The city of Barnes is a small, rural community of approximately 104 residences in Washington County, in north-central Kansas (Figure 2.1). The city lies in a transition zone between the Flint Hills and the glaciated region. The area's topography consists of gently sloping hills of Pleistocene loess ( $<20 \mathrm{ft}$ thick) overlying a shale unit and interbedded shale, limestone, and siltstone of the Permian Chase Group. Groundwater for the Barnes public water supply is produced from the limestone aquifer of the Chase Group.

The city operates two public supply wells (PWS2 and PWS3) in the northwestern portion of the city. Construction details are not available, but KDHE records (KDHE 1997a) indicate that PWS2 has a 10-in. diameter casing and is $155 \mathrm{ft}$ deep, while PWS3 has an 8-in. diameter casing and is $160 \mathrm{ft}$ deep. Historical investigations conducted by the KDHE in 1996-2000 indicated that groundwater flow was generally toward the northeast, ranging from east-northeast to northnortheast.

The CCC/USDA investigations in 2006-2007 (Argonne 2008a) and subsequent extensive monitoring of the potentiometric surface in 2007-2008 (Argonne 2008b-d) showed that pumping of the public water supply wells significantly affects the groundwater flow direction. The continuous monitoring of water levels over an extended period revealed that during periods of pumping, groundwater flows northwestward with an increased gradient. During non-pumping periods, flow is toward the northeast. This cycling significantly affects the movement of the contaminant plume in the subsurface. The result is intermittent drawing of the carbon tetrachloride plume cross-gradient, directly toward wells PWS2 and PWS3.

In its 1996 Phase I comprehensive investigation, the KDHE investigated two source areas for the carbon tetrachloride contamination: (1) the former CCC/USDA grain storage facility and (2) the former high school. The former CCC/USDA grain storage facility was approximately $800 \mathrm{ft}$ east-southeast of the public supply wells (Figure 2.2). The boundaries shown for the former CCC/USDA facility in Figure 2.2 are based on legal descriptions obtained in 2005 (Argonne 2006). Property ownership and lease records were summarized previously (Appendix A in Argonne 2008a). The former school was identified by the KDHE as a potential source area because of documentation regarding the school's storage and use of numerous hazardous chemicals, including carbon tetrachloride and chloroform, together with 
documentation that chemicals were improperly released to the ground outside the school. The CCC/USDA is continuing to evaluate this issue.

Low levels of carbon tetrachloride were initially detected in 1986 in wells PWS2 $(2.1 \mu \mathrm{g} / \mathrm{L})$ and PWS3 $(0.5 \mu \mathrm{g} / \mathrm{L})$. The concentrations detected were well below the drinking water standard of $5.0 \mu \mathrm{g} / \mathrm{L}$ for carbon tetrachloride. From 1986 to July 2008, these wells were sampled 24 times. The maximum carbon tetrachloride concentrations detected in PWS2 and PWS3 were $2.5 \mu \mathrm{g} / \mathrm{L}$ and $2.1 \mu \mathrm{g} / \mathrm{L}$, respectively, in July 1987. In 1988-2008, carbon tetrachloride was detected in well PWS2 in 6 sampling events, with the highest concentration of $1.3 \mu \mathrm{g} / \mathrm{L}$ in August 1996. During the same period, carbon tetrachloride was detected in well PWS3 in only 4 sampling events, with the highest concentration $(1.7 \mu \mathrm{g} / \mathrm{L})$ in July 1999. Carbon tetrachloride was found in both wells at trace concentrations below the method quantitation limit of $1.0 \mu \mathrm{g} / \mathrm{L}$ (estimated at $0.8 \mu \mathrm{g} / \mathrm{L}$ [PWS2] and $0.2 \mu \mathrm{g} / \mathrm{L}$ [PWS3]) during the sampling in July 2008. The historical analytical results for carbon tetrachloride and chloroform in PWS2 and PWS3 are in Table 2.1.

Other documented releases of hazardous chemicals at Barnes include an 18,000-gal spill of nitrate fertilizer from an aboveground storage tank at the Barnes Co-op (KDHE 1990) and leaking underground fuel storage tanks at Westside Service (Figure 2.3). Eight monitoring wells were installed because of the Westside Service release (AEI 2000). Two of these monitoring wells have since been abandoned. 
TABLE 2.1 Analytical results for carbon tetrachloride and chloroform in the public supply wells at Barnes in 1986-2008.

\begin{tabular}{|c|c|c|c|c|}
\hline \multirow{3}{*}{$\begin{array}{c}\text { Sample } \\
\text { Date }\end{array}$} & \multicolumn{4}{|c|}{ Concentration $(\mu \mathrm{g} / \mathrm{L})$} \\
\hline & \multicolumn{2}{|c|}{ Carbon Tetrachloride } & \multicolumn{2}{|c|}{ Chloroform } \\
\hline & PWS2 & PWS3 & PWS2 & PWS3 \\
\hline $4 / 8 / 86$ & 2.1 & 0.5 & ND & ND \\
\hline $4 / 22 / 86$ & 1.3 & 0.2 & ND & ND \\
\hline $7 / 7 / 87$ & 2.5 & 2.1 & ND & ND \\
\hline $1 / 7 / 88$ & $N D^{a}$ & ND & $N R^{b}$ & NR \\
\hline $9 / 2 / 88$ & ND & ND & ND & ND \\
\hline 9/22/88 & ND & ND & NR & NR \\
\hline $1 / 30 / 89$ & ND & ND & 8.7 & 0.8 \\
\hline $7 / 13 / 89$ & ND & ND & NR & 1.5 \\
\hline 8/12/91 & ND & ND & ND & ND \\
\hline 4/11/95 & 0.5 & ND & ND & ND \\
\hline 7/25/95 & 1.1 & ND & ND & ND \\
\hline $5 / 1 / 96$ & ND & ND & ND & ND \\
\hline $8 / 13 / 96^{c}$ & 1.3 & 0.5 & NR & $N R$ \\
\hline $8 / 28 / 97$ & 0.9 & ND & ND & ND \\
\hline $1 / 29 / 99$ & ND & ND & ND & ND \\
\hline $7 / 12 / 99$ & 1.2 & 1.7 & NR & 0.6 \\
\hline $3 / 22 / 00$ & $N D(1)^{d}$ & ND (1) & ND (1) & 1.9 \\
\hline $7 / 15 / 02$ & ND (0.5) & ND (0.5) & NR & NR \\
\hline 7/11/05 & ND (0.5) & ND (0.5) & NR & NR \\
\hline $3 / 9 / 07$ & ND & $0.2 \mathrm{~J}^{\mathrm{e}}$ & ND & ND \\
\hline $4 / 5 / 07$ & ND & ND & ND & ND \\
\hline $11 / 20 / 07$ & ND & ND & ND & ND \\
\hline $3 / 6 / 08$ & ND & ND & ND & ND \\
\hline $7 / 11 / 08$ & $0.8 \mathrm{~J}$ & $0.2 \mathrm{~J}$ & ND & ND \\
\hline
\end{tabular}




\section{TABLE 2.1 (Cont.)}

a ND, not detected.

b NR, result not reported in documents on file.

c Data reported by the KDHE (1997b).

d Detection limit in parentheses.

e Qualifier J indicates an estimated concentration below the purge-and-trap method quantitation limit of $1.0 \mu \mathrm{g} / \mathrm{L}$.

Sources of data (1986-2005): BE\&K 1999, 2000; KDHE 1997b, 2001a, 2006b; PRC 1996. 


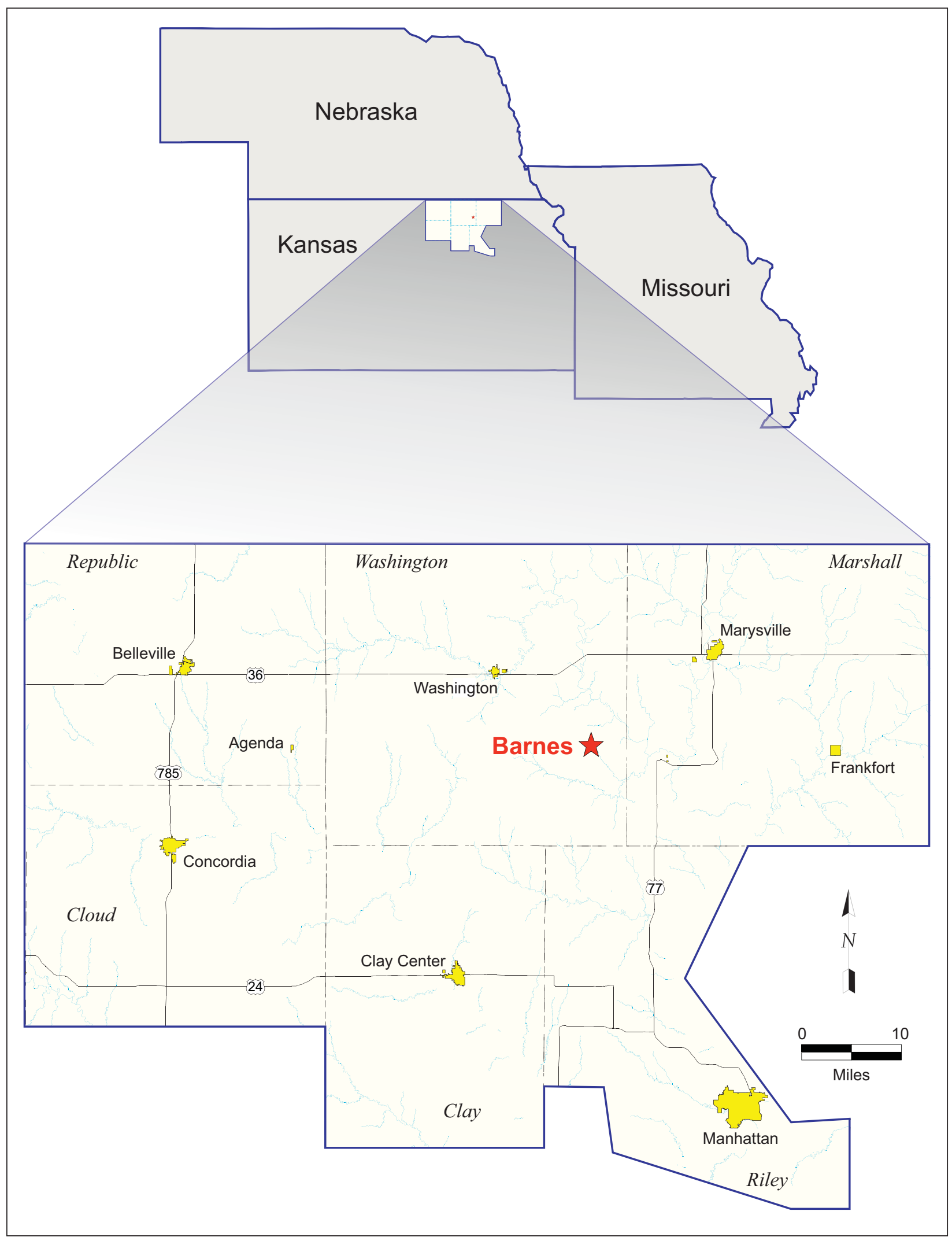

FIGURE 2.1 Location of Washington County and Barnes, Kansas. 


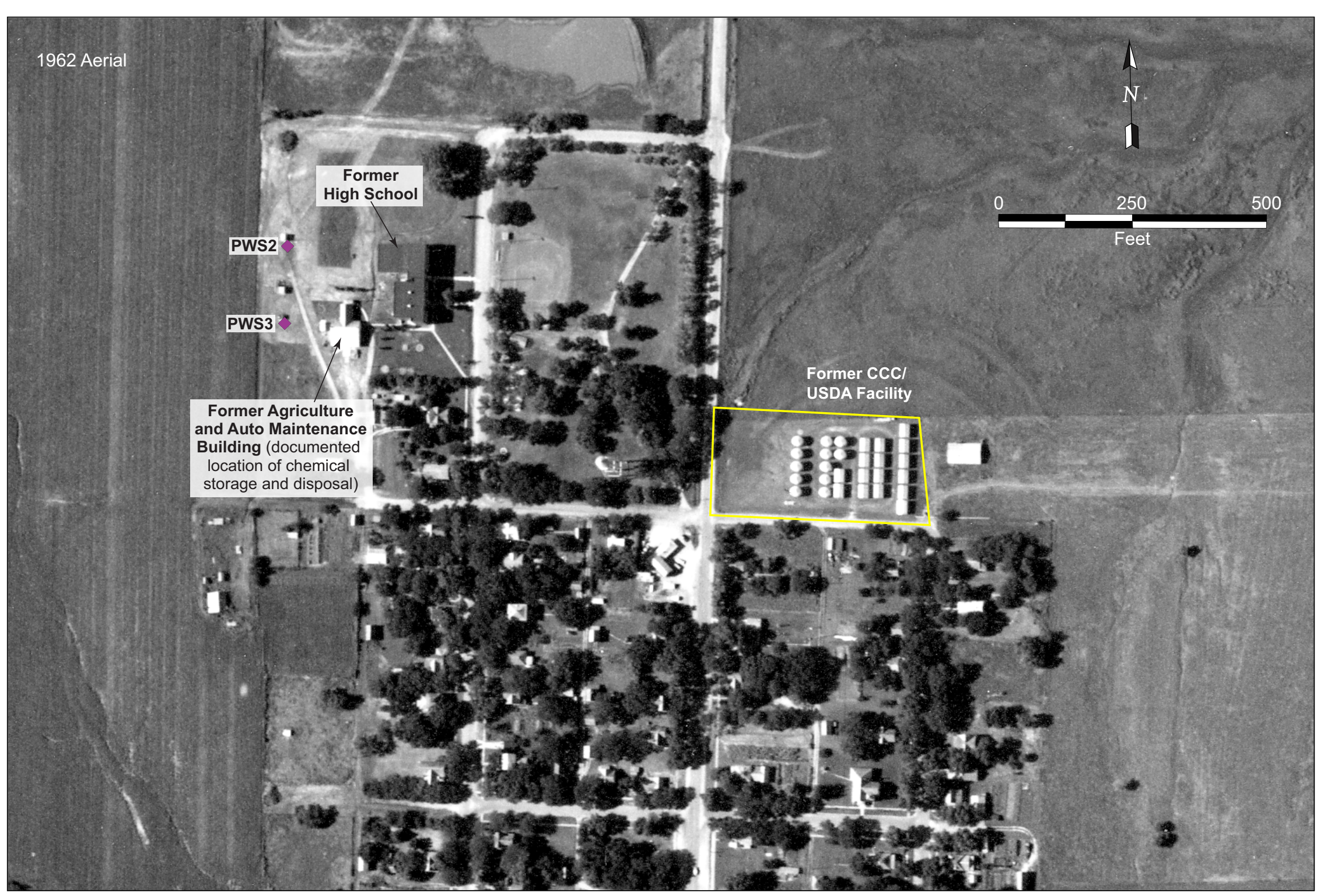

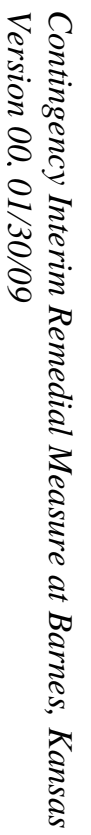

FIGURE 2.2 Grain storage facilities at Barnes in 1962. Source of photograph: USDA (1962). 


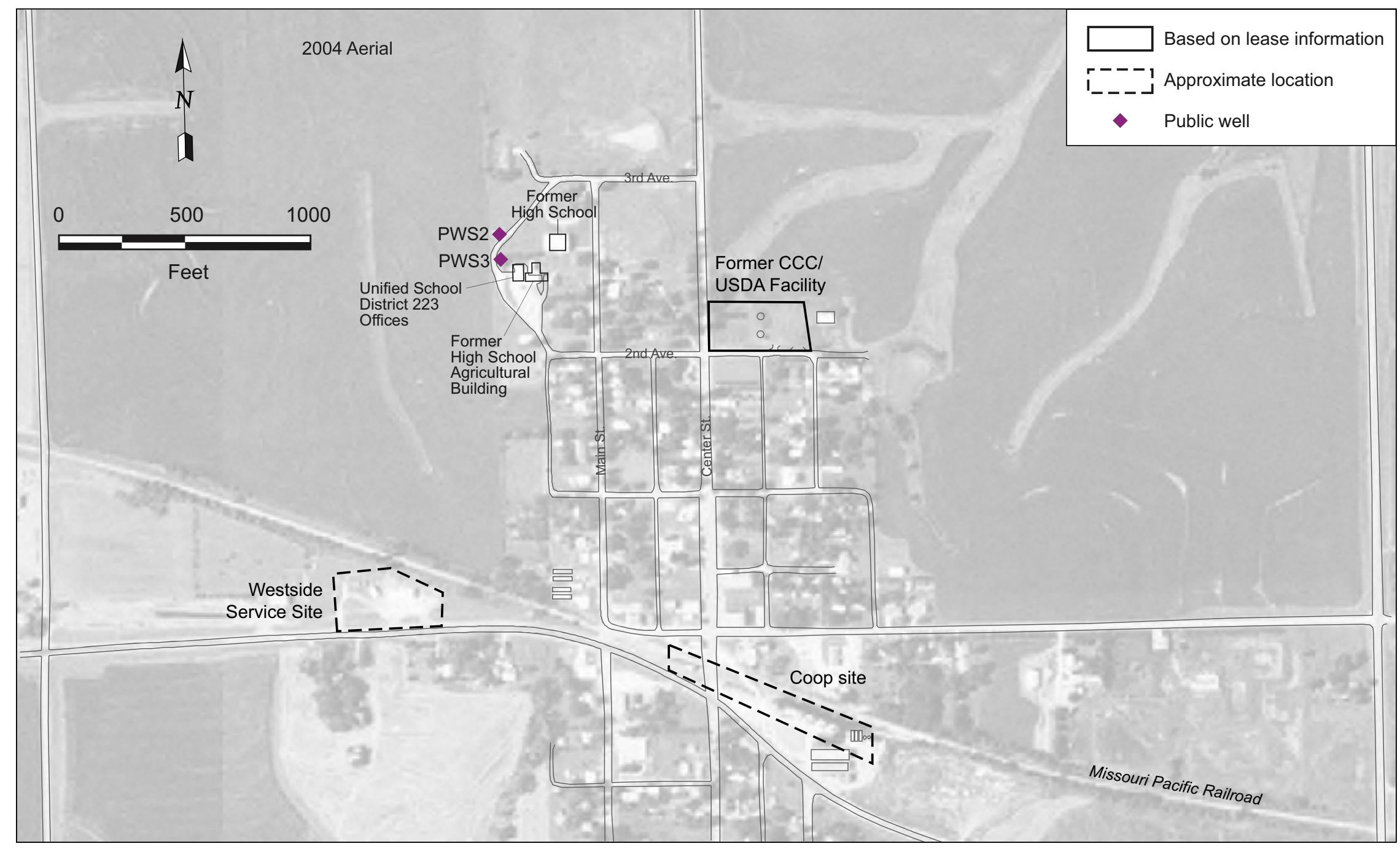

FIGURE 2.3 Location of the former CCC/USDA facility, other relevant structures and facilities, and the public water supply wells at Barnes. Source of photograph: NAIP (2004). 


\section{Summary of Results}

\subsection{Previous Investigations of Carbon Tetrachloride Contamination}

Two previous investigations at Barnes were conducted on behalf of the KDHE. The purposes in both cases were to identify potential sources for the carbon tetrachloride in groundwater and to determine the extent of the contamination.

In 1996, PRC Environmental Management conducted a Phase I comprehensive investigation to identify potential sources for the contamination in wells PWS2 and PWS3 (PRC 1996). Activities focused on the two potential source areas closest to the public supply wells, as follows:

1. The former high school, specifically identified in the comprehensive investigation report prepared for the KDHE (PRC 1996) as an independent potential source of carbon tetrachloride contamination. The PRC (1996) report documented a statement by a lifetime Barnes resident that "chemicals were mixed in the agricultural building as part of the high school curriculum and then dumped outside." The report (PRC 1996) further documented a transmittal from the high school superintendent to the KDHE regarding proper disposal of chemicals stored at the high school (Unified School District 223), including two pints of carbon tetrachloride (USD 223 1989). The site of the former high school, where carbon tetrachloride is known to have been present in 1989, is less than $250 \mathrm{ft}$ from the public wells (Appendix A).

2. The site of the former CCC/USDA grain storage facility, approximately $800 \mathrm{ft}$ east-southeast from the public wells. The CCC/USDA formerly operated a grain storage facility in Barnes, approximately $800 \mathrm{ft}$ east-southeast of the public water supply wells. Carbon tetrachloride was used in the treatment of grain.

As part of the 1996 comprehensive investigation for the KDHE, shallow soil gas and soil samples (7-24 ft BGL) were collected (April 29, 1996, through May 1, 1996) at the sites of the former high school and the former CCC/USDA grain storage facility (PRC 1996). Groundwater samples were also collected from the public water supply wells and local private wells. All 
samples were analyzed for carbon tetrachloride and chloroform. Low levels of carbon tetrachloride and chloroform were detected in soil gas samples collected at both the former high school and the former CCC/USDA grain storage facility. In off-site laboratory analysis of one soil gas sample from each location, the low-level carbon tetrachloride contamination was confirmed. In addition, BTEX (benzene, toluene, ethylbenzene, xylene) contamination was identified in the soil gas sample from the former CCC/USDA facility. No carbon tetrachloride or chloroform was present above the detection limit in any of the groundwater samples collected in April-May 1996, including those from PWS2 and PWS3 (Table 2.1 and Appendix B, Table B.1).

In 1998-1999, BE\&K/Terranext conducted a Phase II comprehensive investigation on behalf of the KDHE to determine the extent of the groundwater contamination and the local groundwater gradient (BE\&K 1999). Five monitoring wells - MW1S, MW1D, MW2D, MW3D, and MW4D - were installed to delineate the contamination previously detected in wells PWS2 and PWS3 (Figure 3.1). Wells MW1D, MW2D, MW3D, and MW4D were screened at approximately 100-160 ft BGL (below ground level) to intersect the water-bearing zone in which the public supply wells are screened (Appendix B, Table B.2). Carbon tetrachloride was detected in MW4D (on the former CCC/USDA property) at concentrations of $4.0 \mu \mathrm{g} / \mathrm{L}$ and $3.9 \mu \mathrm{g} / \mathrm{L}$ in December 1998 and January 1999, respectively (Appendix B, Table B.1). Carbon tetrachloride was not detected in any of the other monitoring wells in the area in these sampling events.

In the 1999 comprehensive investigation conducted for the KDHE, the groundwater gradient was determined to be toward the north-northeast, placing the former CCC/USDA facility downgradient from or cross-gradient to the two public supply wells (BE\&K 1999). The two public supply wells and the five monitoring wells were sampled again on behalf of the KDHE in March 2000 (BE\&K 2000). Carbon tetrachloride was again detected in MW4D, at a concentration of $5.1 \mu \mathrm{g} / \mathrm{L}$. Carbon tetrachloride was not detected in any of the other monitoring wells (Appendix B, Table B.1) or the public supply wells (Table 2.1). During the 2000 sampling event, the groundwater gradient was determined to be to the east-northeast, verifying the interpretation made in the 1999 investigation that the former CCC/USDA facility was downgradient from the public supply wells (BE\&K 2000).

In 2006 and 2007, Argonne conducted a comprehensive investigation of the Barnes site (Argonne 2008a). The effort included the installation of 23 monitoring wells at 13 distinct locations. The wells were completed at depths ranging from $50 \mathrm{ft}$ to $137 \mathrm{ft}$ BGL and screened to 
intersect lithologic zones that indicated the presence of water. The analytical results for groundwater indicated trace to moderate concentrations of carbon tetrachloride and chloroform on the former CCC/USDA property and at locations throughout the investigation area (Figures 3.2 and 3.3). Subsequent monitoring of the wells in 2007 and 2008 (Argonne 2008b-d) indicated similar results for groundwater (Figures 3.4 and 3.5).

Complete historical analytical results for sampling events at Barnes in 1986-2005 are in Table B.1, Appendix B. Analytical data acquired by the CCC/USDA in 2006-2008 are in Table 3.1. All available construction details for the wells sampled as part of the historical carbon tetrachloride investigations, as well as those sampled as part of the Westside Service investigation and those installed by the CCC/USDA in 2007, are in Table B.2, Appendix B.

\subsection{Geologic and Hydrogeologic Conditions}

Barnes lies in a transition zone between the Flint Hills and the glaciated region. The area's topography consists of gently sloping hills of Pleistocene loess (with variations in elevation $<50 \mathrm{ft}$ ) overlying a shale unit and interbedded shale, limestone, and siltstone of the Permian Chase Group. Groundwater for the public water supply is produced from the bedrock aquifer of the Chase Group.

The site lithology was identified through the collection of core samples from monitoring wells MW5-MW17. Four stratigraphic cross sections were developed from the lithologic descriptions. The locations are shown in Figure 3.6. The cross sections are included as Figures 3.7-3.10.

The predominant lithology consists of a thin layer of silty clay to clayey silt with fine sand in the upper 2-20 ft. This layer is underlain by highly weathered shale interbedded with thin layers of fractured limestone at depths ranging from approximately $18 \mathrm{ft}$ BGL at MW8 to $132 \mathrm{ft}$ BGL at MW13. Groundwater was encountered predominantly in the limestone layers. Monitoring wells were screened in the limestone layers in all wells except MW14 and MW15. These two locations did not exhibit limestone at depths greater than $58.5 \mathrm{ft} \mathrm{BGL}$.

The monitoring wells installed during the 2006-2007 investigation were screened at various depths, with several locations being completed as nested wells to enable determination of contaminant migration pathways and contaminant concentrations in groundwater at multiple 
depths where the presence of a water-bearing zone was indicated. The stratigraphic cross sections (Figures 3.7-3.10) depict the screened depths in the monitoring wells.

The designations “S," "M," and " $\mathrm{D}$ ” in monitoring well names were assigned the time of well installation. They indicate shallow, medium, and deep screens (relative depths) in an individual well, rather than the aquifer zone screened. Detailed evaluation of the hand-measured water levels in March 2008 and July 2008 (Table 3.2) and the contemporaneous concentration data for volatile organic compounds (VOCs) suggested that at least three vertically distinct zones are present in the aquifer. The aquifer zones are as follows:

- Shallow Aquifer Zone. The only monitoring wells believed to be completed in the shallow zone are MW1S, MW11S, and MW12S. Wells MW1S and MW12S were dry during the March and July 2008 sampling events; well MW11S showed a water level elevation of approximately 1,312 ft AMSL (above mean sea level) in July 2008.

- Intermediate Aquifer Zone. The intermediate zone is believed to be represented by monitoring wells MW10S, MW11M, MW12M, MW13S, and MW17. Water level elevations measured manually for these wells in July 2008 were approximately 1,255-1,258 ft AMSL.

- Deep Aquifer Zone. Monitoring wells believed to represent the deep zone include MW1D, MW2D, MW3D, MW4D, MW5, MW6S, MW6D, MW7, MW8, MW9, MW10D, MW11D, MW12D, MW13D, MW14S, MW14D, MW15S, MW15D, MW16S, and MW16D. The water level elevations measured manually in these monitoring wells in July 2008 were approximately 1,229-1,239 ft AMSL. On the basis of its hand-measured water level in November 2007 (approximately 1,220 ft AMSL [Table 3.2] and longterm automatic measurements [Supplement 1 in Argonne 2008b], the Oentrich well is also considered to be screened in the deep aquifer zone. All of the wells equipped with data loggers for water level monitoring are screened in the deep and intermediate zones. Data loggers were added to the five intermediate-depth monitoring wells (MW10S, MW11M, MW12M, MW13S, and MW17) on November 18, 2008. 
The vertical distribution of the carbon tetrachloride in groundwater indicates that the highest concentrations have consistently been detected in the intermediate zone, at wells MW10S (11-56 $\mu \mathrm{g} / \mathrm{L})$, MW12M (18-27 $\mu \mathrm{g} / \mathrm{L})$, and MW13S (17-21 $\mu \mathrm{g} / \mathrm{L})$ (Figure 3.11 and Table 3.1). The deep-zone wells at these locations showed lower concentrations (3.9-6.3 $\mu \mathrm{g} / \mathrm{L}$ at MW10D, $<1.0-1.6 \mu \mathrm{g} / \mathrm{L}$ at MW12D, and 5.9-11 $\mu \mathrm{g} / \mathrm{L}$ at MW13D) (Figure 3.12 and Table 3.1). The only exception was deep well MW8, which has shown carbon tetrachloride concentrations of 14-23 $\mu \mathrm{g} / \mathrm{L}$ (Figure 3.12 and Table 3.1). There is no intermediate well at this location. The shallow-zone well (MW11S) continued to show no detectable concentrations of carbon tetrachloride (Table 3.1).

Drawdown was observed in all monitoring wells when the public water supply wells were pumping. This phenomenon is illustrated for the months of January-June 2008 by the hydrographs in Figure 3.13. Also illustrated by these hydrographs is a consistent rise in the water table of approximately 6.5-11 ft in January-June 2008.

The historical data for existing KDHE monitoring wells indicated a northeasterly groundwater flow direction (BE\&K 1999, 2000). In contrast, the groundwater levels measured by data loggers in 2007 and 2008 indicate northwesterly flow when the public water supply wells are pumping, with apparently stronger gradients. Under non-pumping conditions, the flow between the former CCC/USDA property and the public wells appears to be somewhat radial, away from an area of relatively higher water levels across the southwest and central portions of the town. Under these conditions, the hydraulic gradients, especially across the central part of the study area, are quite low, and the apparent flow direction varies from location to location. These results suggest that, at least locally, the apparent flow direction toward the northeast - proposed on the basis of work conducted for the KDHE in 1999-2000 — is possible, though transient. Nevertheless, the 2007 and 2008 results indicate an apparent gradient that is stronger and much more consistently toward the pumping wells when they are operating. Figures 3.14 and 3.15 depict interpreted groundwater flow directions in 2007 and 2008, before and during pumping episodes.

Relatively little movement of the contaminant plume in the area between the public wells and the former CCC/USDA property might be expected under non-pumping conditions, though the variable low gradients at these times could, in part, account for the relatively broad distribution of low carbon tetrachloride concentrations along the flanks of the main migration pathway toward the public wells. 
TABLE 3.1 Analytical results for volatile organic compounds in groundwater samples collected at Barnes in July 2006 to July 2008.

\begin{tabular}{|c|c|c|c|c|c|c|}
\hline \multirow[b]{2}{*}{ Location } & \multirow[b]{2}{*}{$\begin{array}{l}\text { Screen } \\
\text { Interval } \\
\text { (ft BGL) }\end{array}$} & \multirow[b]{2}{*}{ Sample } & \multirow[b]{2}{*}{$\begin{array}{l}\text { Sample } \\
\text { Date }\end{array}$} & \multicolumn{3}{|c|}{ Concentration $(\mu \mathrm{g} / \mathrm{L})$} \\
\hline & & & & $\begin{array}{c}\text { Carbon } \\
\text { Tetrachloride }\end{array}$ & Chloroform & $\begin{array}{l}\text { Methylene } \\
\text { Chloride }\end{array}$ \\
\hline \multicolumn{7}{|c|}{ Previously existing KDHE monitoring wells } \\
\hline \multirow[t]{5}{*}{ MW1S } & $13.3-23.3$ & Not sampled (well dry) & $7 / 19 / 06$ & - & - & - \\
\hline & & Not sampled (well dry) & $4 / 4 / 07$ & - & - & - \\
\hline & & Not sampled (well dry) & $11 / 18 / 07$ & - & - & - \\
\hline & & Not sampled (well dry) & $3 / 4 / 08$ & - & - & - \\
\hline & & Not sampled (well dry) & $7 / 9 / 08$ & - & - & - \\
\hline \multirow[t]{5}{*}{ MW1D } & $139.85-159.4$ & BAMW1D-W-21688 & 7/19/06 & 1.0 & $N D^{a}$ & ND \\
\hline & & BAMW1D-W-22565 & $4 / 4 / 07$ & 1.2 & ND & ND \\
\hline & & BAMW1D-W-22593 & $11 / 18 / 07$ & ND & ND & ND \\
\hline & & BAMW1D-W-22627 & $3 / 4 / 08$ & $0.2 \mathrm{~J}^{\mathrm{b}}$ & ND & ND \\
\hline & & BAMW1D-W-22668 & $7 / 9 / 08$ & $0.2 \mathrm{~J}$ & ND & ND \\
\hline \multirow[t]{5}{*}{ MW2D } & $133.26-152.93$ & BAMW2D-W-21687 & 7/19/06 & ND & ND & ND \\
\hline & & BAMW2D-W-22564 & $4 / 4 / 07$ & ND & ND & ND \\
\hline & & BAMW2D-W-22594 & $11 / 18 / 07$ & ND & ND & ND \\
\hline & & BAMW2D-W-22628 & $3 / 7 / 08$ & ND & ND & ND \\
\hline & & BAMW2D-W-22669 & $7 / 10 / 08$ & ND & ND & ND \\
\hline \multirow[t]{5}{*}{ MW3D } & $133.02-152.73$ & BAMW3D-W-21686 & 7/19/06 & ND & ND & ND \\
\hline & & BAMW3D-W-22567 & $4 / 4 / 07$ & ND & ND & ND \\
\hline & & BAMW3D-W-22595 & $11 / 19 / 07$ & ND & ND & ND \\
\hline & & BAMW3D-W-22629 & $3 / 7 / 08$ & ND & ND & ND \\
\hline & & BAMW3D-W-22670 & $7 / 10 / 08$ & ND & ND & ND \\
\hline \multirow[t]{5}{*}{ MW4D } & $98.38-118.22$ & BAMW4D-W-21690 & $7 / 20 / 06$ & 2.1 & ND & ND \\
\hline & & BAMW4D-W-22583 & $4 / 6 / 07$ & 3.5 & $0.1 \mathrm{~J}$ & ND \\
\hline & & BAMW4D-W-22596 & $11 / 19 / 07$ & 1.7 & $0.4 \mathrm{~J}$ & ND \\
\hline & & BAMW4D-W-22642 & $3 / 9 / 08$ & 18 & $0.4 \mathrm{~J}$ & ND \\
\hline & & BAMW4D-W-22671 & $7 / 12 / 08$ & 9.4 & $0.5 \mathrm{~J}$ & ND \\
\hline \multicolumn{7}{|c|}{ CCC/USDA wells installed during the 2006-2007 investigation } \\
\hline \multirow[t]{4}{*}{ MW5 } & $110-120$ & BAMW5-W-22589 & $4 / 6 / 07$ & $0.6 \mathrm{~J}$ & ND & ND \\
\hline & & BAMW5-W-22597 & $11 / 19 / 07$ & $0.6 \mathrm{~J}$ & ND & ND \\
\hline & & BAMW5-W-22637 & $3 / 8 / 08$ & $0.7 \mathrm{~J}$ & ND & ND \\
\hline & & BAMW5-W-22672 & $7 / 11 / 08$ & ND & ND & ND \\
\hline \multirow[t]{4}{*}{ MW6S } & $90.5-100.5$ & Not sampled (well dry) & $4 / 4 / 07$ & & & \\
\hline & & BAMW6S-W-22598 & $11 / 19 / 07$ & $0.3 \mathrm{~J}$ & ND & ND \\
\hline & & BAMW6S-W-22635 & $3 / 8 / 08$ & $0.4 \mathrm{~J}$ & ND & ND \\
\hline & & BAMW6S-W-22673 & $7 / 11 / 08$ & ND & ND & ND \\
\hline \multirow[t]{4}{*}{ MW6D } & $105-115$ & BAMW6D-W-22573 & $4 / 5 / 07$ & ND & ND & ND \\
\hline & & BAMW6D-W-22599 & $11 / 19 / 07$ & $0.5 \mathrm{~J}$ & ND & ND \\
\hline & & BAMW6D-W-22636 & $3 / 8 / 08$ & $0.8 \mathrm{~J}$ & ND & ND \\
\hline & & BAMW6D-W-22674 & $7 / 11 / 08$ & $0.9 \mathrm{~J}$ & ND & ND \\
\hline
\end{tabular}


TABLE 3.1 (Cont.)

\begin{tabular}{|c|c|c|c|c|c|c|}
\hline \multirow[b]{2}{*}{ Location } & \multirow[b]{2}{*}{$\begin{array}{l}\text { Screen } \\
\text { Interval } \\
\text { (ft BGL) }\end{array}$} & \multirow[b]{2}{*}{ Sample } & \multirow[b]{2}{*}{$\begin{array}{l}\text { Sample } \\
\text { Date }\end{array}$} & \multicolumn{3}{|c|}{ Concentration $(\mu / L)$} \\
\hline & & & & $\begin{array}{c}\text { Carbon } \\
\text { Tetrachloride }\end{array}$ & Chloroform & $\begin{array}{c}\text { Methylene } \\
\text { Chloride }\end{array}$ \\
\hline \multicolumn{7}{|c|}{ CCC/USDA wells installed during the 2006-2007 investigation (cont.) } \\
\hline \multirow[t]{4}{*}{ MW7 } & $116-126$ & BAMW7-W-22588 & $4 / 6 / 07$ & 1.0 & ND & ND \\
\hline & & BAMW7-W-22600 & $11 / 19 / 07$ & 2.6 & ND & ND \\
\hline & & BAMW7-W-22643 & 3/9/08 & 2.8 & ND & ND \\
\hline & & BAMW7-W-22675 & $7 / 12 / 08$ & 1.7 & ND & ND \\
\hline \multirow[t]{4}{*}{ MW8 } & $110-120$ & BAMW8-W-22584 & $4 / 6 / 07$ & 14 & $0.7 \mathrm{~J}$ & ND \\
\hline & & BAMW8-W-22601 & $11 / 19 / 07$ & 23 & $0.6 \mathrm{~J}$ & ND \\
\hline & & BAMW8-W-22652 & 3/10/08 & 19 & $0.6 \mathrm{~J}$ & ND \\
\hline & & BAMW8-W-22676 & $7 / 11 / 08$ & 21 & $0.6 \mathrm{~J}$ & ND \\
\hline \multirow[t]{4}{*}{ MW9 } & $100-110$ & BAMW9-W-22582 & $4 / 5 / 07$ & 1.0 & ND & ND \\
\hline & & BAMW9-W-22602 & $11 / 19 / 07$ & 7.7 & $0.6 \mathrm{~J}$ & ND \\
\hline & & BAMW9-W-22647 & $3 / 9 / 08$ & 3.0 & $0.3 \mathrm{~J}$ & ND \\
\hline & & BAMW9-W-22678 & 7/11/08 & 1.3 & $0.3 \mathrm{~J}$ & ND \\
\hline \multirow[t]{4}{*}{ MW10S } & $93-103$ & BAMW10S-W-22586 & $4 / 6 / 07$ & 20 & 1.4 & ND \\
\hline & & BAMW10S-W-22603 & $11 / 19 / 07$ & 11 & $0.7 \mathrm{~J}$ & ND \\
\hline & & BAMW10S-W-22649 & 3/10/08 & 56 & 2 & ND \\
\hline & & BAMW10S-W-22679 & $7 / 11 / 08$ & 49 & 1.8 & ND \\
\hline \multirow[t]{4}{*}{ MW10D } & $115-125$ & BAMW10D-W-22585 & $4 / 6 / 07$ & 2.4 & $0.2 \mathrm{~J}$ & ND \\
\hline & & BAMW10D-W-22604 & $11 / 19 / 07$ & 6.3 & $0.5 \mathrm{~J}$ & ND \\
\hline & & BAMW10D-W-22646 & $3 / 9 / 08$ & 5.7 & $0.5 \mathrm{~J}$ & ND \\
\hline & & BAMW10D-W-22680 & $7 / 11 / 08$ & 3.9 & $0.7 \mathrm{~J}$ & ND \\
\hline \multirow[t]{4}{*}{ MW11S } & $40-50$ & BAMW11S-W-22570 & $4 / 4 / 07$ & ND & 1.1 & ND \\
\hline & & BAMW11S-W-22605 & $11 / 19 / 07$ & ND & $0.6 \mathrm{~J}$ & ND \\
\hline & & BAMW11S-W-22630 & $3 / 5 / 08$ & ND & $0.6 \mathrm{~J}$ & ND \\
\hline & & BAMW11S-W-22681 & $7 / 10 / 08$ & ND & $0.4 \mathrm{~J}$ & ND \\
\hline \multirow[t]{4}{*}{ MW11M } & $90-100$ & BAMW11M-W-22572 & $4 / 5 / 07$ & ND & ND & ND \\
\hline & & BAMW11M-W-22606 & $11 / 19 / 07$ & 3.7 & ND & ND \\
\hline & & BAMW11M-W-22644 & $3 / 6 / 08$ & 2.4 & $0.5 \mathrm{~J}$ & ND \\
\hline & & BAMW11M-W-22682 & $7 / 10 / 08$ & 2.4 & $0.7 \mathrm{~J}$ & ND \\
\hline \multirow[t]{4}{*}{ MW11D } & $125-135$ & BAMW11D-W-22571 & $4 / 4 / 07$ & 1.1 & ND & ND \\
\hline & & BAMW11D-W-22607 & $11 / 19 / 07$ & $0.8 \mathrm{~J}$ & ND & ND \\
\hline & & BAMW11D-W-22639 & $3 / 5 / 08$ & $0.4 \mathrm{~J}$ & ND & ND \\
\hline & & BAMW11D-W-22683 & $7 / 10 / 08$ & $0.9 \mathrm{~J}$ & ND & ND \\
\hline \multirow[t]{4}{*}{ MW12S } & $43-50$ & Not sampled (well dry) & $4 / 5 / 07$ & & & \\
\hline & & Not sampled (well dry) & $11 / 19 / 07$ & & & \\
\hline & & Not sampled (well dry) & 3/10/08 & & & \\
\hline & & Not sampled (well dry) & $7 / 10 / 08$ & & & \\
\hline \multirow[t]{4}{*}{ MW12M } & $90-100$ & BAMW12M-W-22580 & $4 / 5 / 07$ & 20 & 4.2 & ND \\
\hline & & BAMW12M-W-22609 & $11 / 19 / 07$ & 18 & 5.1 & ND \\
\hline & & BAMW12M-W-22651 & 3/10/08 & 18 & 2.6 & ND \\
\hline & & BAMW12M-W-22685 & $7 / 10 / 08$ & 27 & 4.2 & ND \\
\hline
\end{tabular}


TABLE 3.1 (Cont.)

\begin{tabular}{|c|c|c|c|c|c|c|}
\hline \multirow[b]{2}{*}{ Location } & \multirow[b]{2}{*}{$\begin{array}{l}\text { Screen } \\
\text { Interval } \\
\text { (ft BGL) }\end{array}$} & \multirow[b]{2}{*}{ Sample } & \multirow[b]{2}{*}{$\begin{array}{l}\text { Sample } \\
\text { Date }\end{array}$} & \multicolumn{3}{|c|}{ Concentration $(\mu / L)$} \\
\hline & & & & $\begin{array}{c}\text { Carbon } \\
\text { Tetrachloride }\end{array}$ & Chloroform & $\begin{array}{l}\text { Methylene } \\
\text { Chloride }\end{array}$ \\
\hline \multicolumn{7}{|c|}{ CCC/USDA wells installed during the 2006-2007 investigation (cont.) } \\
\hline \multirow[t]{4}{*}{ MW12D } & $115-125$ & BAMW12D-W-22576 & $4 / 5 / 07$ & $0.6 \mathrm{~J}$ & ND & ND \\
\hline & & BAMW12D-W-22610 & $11 / 18 / 07$ & 1.6 & ND & ND \\
\hline & & BAMW12D-W-22641 & $3 / 9 / 08$ & 1.0 & ND & ND \\
\hline & & BAMW12D-W-22686 & $7 / 11 / 08$ & $0.7 \mathrm{~J}$ & ND & ND \\
\hline \multirow[t]{4}{*}{ MW13S } & $112-122$ & BAMW13S-W-22575 & $4 / 5 / 07$ & 21 & 1.6 & ND \\
\hline & & BAMW13S-W-22611 & $11 / 19 / 07$ & 17 & 1.8 & ND \\
\hline & & BAMW13S-W-22650 & $3 / 10 / 08$ & 17 & 1.5 & ND \\
\hline & & BAMW13S-W-22687 & $7 / 9 / 08$ & 17 & 1.9 & ND \\
\hline \multirow[t]{4}{*}{ MW13D } & $127-137$ & BAMW13D-W-22574 & $4 / 5 / 07$ & 3.5 & $0.4 \mathrm{~J}$ & ND \\
\hline & & BAMW13D-W-22612 & $11 / 19 / 07$ & 5.9 & $0.2 \mathrm{~J}$ & ND \\
\hline & & BAMW13D-W-22645 & $3 / 9 / 08$ & 11 & 1.1 & ND \\
\hline & & BAMW13D-W-22688 & $7 / 9 / 08$ & 5.9 & $0.9 \mathrm{~J}$ & ND \\
\hline \multirow[t]{4}{*}{ MW14S } & $108-118$ & BAMW14S-W-22569 & $4 / 4 / 07$ & $0.9 \mathrm{~J}$ & ND & ND \\
\hline & & BAMW14S-W-22613 & $11 / 18 / 07$ & 1.2 & ND & ND \\
\hline & & BAMW14S-W-22640 & $3 / 8 / 08$ & 4.3 & $0.3 \mathrm{~J}$ & ND \\
\hline & & BAMW14S-W-22689 & $7 / 10 / 08$ & 5.6 & $0.3 \mathrm{~J}$ & ND \\
\hline \multirow[t]{4}{*}{ MW14D } & $123-133$ & BAMW14D-W-22568 & $4 / 4 / 07$ & 1.2 & ND & ND \\
\hline & & BAMW14D-W-22614 & $11 / 18 / 07$ & $0.6 \mathrm{~J}$ & ND & ND \\
\hline & & BAMW14D-W-22638 & $3 / 8 / 08$ & $0.7 \mathrm{~J}$ & ND & ND \\
\hline & & BAMW14D-W-22690 & $7 / 10 / 08$ & $0.5 \mathrm{~J}$ & ND & ND \\
\hline \multirow[t]{4}{*}{ MW15S } & $88-98$ & BAMW15S-W-22560 & $4 / 4 / 07$ & 1.5 & ND & ND \\
\hline & & BAMW15S-W-22615 & $11 / 18 / 07$ & 8.7 & $0.4 \mathrm{~J}$ & ND \\
\hline & & BAMW15S-W-22648 & $3 / 10 / 08$ & 1.8 & $0.2 \mathrm{~J}$ & ND \\
\hline & & BAMW15S-W-22691 & $7 / 12 / 08$ & 2.2 & $0.3 \mathrm{~J}$ & ND \\
\hline \multirow[t]{4}{*}{ MW15D } & $105-115$ & BAMW15D-W-22561 & $4 / 4 / 07$ & ND & ND & ND \\
\hline & & BAMW15D-W-22616 & $11 / 18 / 07$ & ND & ND & ND \\
\hline & & BAMW15D-W-22631 & $3 / 8 / 08$ & $0.2 \mathrm{~J}$ & ND & ND \\
\hline & & BAMW15D-W-22692 & $7 / 12 / 08$ & ND & ND & ND \\
\hline \multirow[t]{4}{*}{ MW16S } & $76-86$ & BAMW16S-W-22563 & $4 / 4 / 07$ & ND & ND & ND \\
\hline & & BAMW16S-W-22617 & $11 / 19 / 07$ & ND & ND & ND \\
\hline & & BAMW16S-W-22632 & $3 / 7 / 08$ & $0.4 \mathrm{~J}$ & ND & ND \\
\hline & & BAMW16S-W-22693 & $7 / 11 / 08$ & ND & ND & ND \\
\hline \multirow[t]{4}{*}{ MW16D } & $90-100$ & BAMW16D-W-22562 & $4 / 4 / 07$ & ND & ND & ND \\
\hline & & BAMW16D-W-22618 & $11 / 19 / 07$ & ND & ND & ND \\
\hline & & BAMW16D-W-22633 & $3 / 7 / 08$ & ND & ND & ND \\
\hline & & BAMW16D-W-22694 & $7 / 11 / 08$ & ND & ND & ND \\
\hline \multirow[t]{4}{*}{ MW17 } & $120-130$ & BAMW17D-W-22566 & $4 / 4 / 07$ & ND & ND & ND \\
\hline & & BAMW17D-W-22619 & $11 / 19 / 07$ & ND & ND & ND \\
\hline & & BAMW17-W-22634 & $3 / 5 / 08$ & $0.3 \mathrm{~J}$ & ND & ND \\
\hline & & BAMW17-W-22695 & $7 / 9 / 08$ & $0.4 \mathrm{~J}$ & ND & ND \\
\hline
\end{tabular}


TABLE 3.1 (Cont.)

\begin{tabular}{|c|c|c|c|c|c|c|}
\hline \multirow[b]{2}{*}{ Location } & \multirow[b]{2}{*}{$\begin{array}{l}\text { Screen } \\
\text { Interval } \\
\text { (ft BGL) }\end{array}$} & \multirow[b]{2}{*}{ Sample } & \multirow[b]{2}{*}{$\begin{array}{l}\text { Sample } \\
\text { Date }\end{array}$} & \multicolumn{3}{|c|}{ Concentration $(\mu / L)$} \\
\hline & & & & $\begin{array}{c}\text { Carbon } \\
\text { Tetrachloride }\end{array}$ & Chloroform & $\begin{array}{l}\text { Methylene } \\
\text { Chloride }\end{array}$ \\
\hline \multicolumn{7}{|c|}{ Private wells } \\
\hline \multirow[t]{6}{*}{ Oentrich } & 150 & BAOENT-W-21693 & 7/20/06 & $0.3 \mathrm{~J}$ & ND & ND \\
\hline & & BAOENT-W-21713 & $8 / 2 / 06$ & $0.6 \mathrm{~J}$ & ND & ND \\
\hline & & BAOENTRICH-W-22579 & $4 / 5 / 07$ & $0.6 \mathrm{~J}$ & ND & ND \\
\hline & & BAOENTRICH-W-22622 & $11 / 19 / 07$ & $0.8 \mathrm{~J}$ & ND & ND \\
\hline & & BAOENTRICH-W-22654 & $3 / 6 / 08$ & 1.3 & ND & ND \\
\hline & & BAOENTRICH-W-22695 & $7 / 11 / 08$ & $0.3 \mathrm{~J}$ & ND & ND \\
\hline \multirow{2}{*}{ Sedivy } & 138 & BACW-W-21849 & $8 / 22 / 06$ & ND & ND & ND \\
\hline & & BASED2-W-21913 & 9/13/06 & ND & ND & ND \\
\hline Sedivy1 & 90 & Not sampled (well dry) & 9/13/06 & - & - & - \\
\hline \multicolumn{7}{|c|}{ Public water supply wells } \\
\hline \multirow[t]{5}{*}{ PWS2 } & 155 & BAPWS2-W-22510 & $3 / 9 / 07$ & ND & ND & ND \\
\hline & & BAPW2-W-22578 & $4 / 5 / 07$ & ND & ND & ND \\
\hline & & BAPW2-W-22620 & $11 / 20 / 07$ & ND & ND & ND \\
\hline & & BAPWS2-W-22655 & $3 / 6 / 08$ & ND & ND & ND \\
\hline & & BAPWS2-W-22696 & 7/11/08 & $0.8 \mathrm{~J}$ & ND & ND \\
\hline \multirow[t]{5}{*}{ PWS3 } & 160 & BAPWS3-W-22511 & $3 / 9 / 07$ & $0.2 \mathrm{~J}$ & ND & ND \\
\hline & & BAPW3-W-22577 & $4 / 5 / 07$ & ND & ND & ND \\
\hline & & BAPW3-W-22621 & $11 / 20 / 07$ & ND & ND & ND \\
\hline & & BAPWS3-W-22656 & $3 / 6 / 08$ & ND & ND & ND \\
\hline & & BAPWS3-W-22697 & $7 / 11 / 08$ & $0.2 \mathrm{~J}$ & ND & ND \\
\hline
\end{tabular}

a ND, contaminant not detected at an instrument detection limit of $0.1 \mu \mathrm{g} / \mathrm{L}$.

b Qualifier J indicates an estimated concentration below the purge-and-trap method quantitation limit of $1.0 \mu \mathrm{g} / \mathrm{L}$. 
TABLE 3.2 Hand-measured water levels at Barnes in November 2007 to July 2008.

\begin{tabular}{|c|c|c|c|c|c|c|c|c|c|}
\hline \multirow[b]{3}{*}{ Well } & \multirow{3}{*}{$\begin{array}{l}\text { Reference } \\
\text { Elevation } \\
\text { (ft AMSL) }\end{array}$} & \multicolumn{8}{|c|}{ Water Level on Date Indicated } \\
\hline & & \multicolumn{2}{|c|}{ 11/18-19/07 } & \multicolumn{2}{|c|}{$12 / 19 / 07$} & \multicolumn{2}{|c|}{$3 / 4-10 / 08$} & \multicolumn{2}{|c|}{$7 / 9-12 / 08$} \\
\hline & & $\mathrm{ft}$ TOC $^{\mathrm{a}}$ & $\mathrm{ft}$ AMSL & $\mathrm{ft} \mathrm{TOC}{ }^{\mathrm{a}}$ & $\mathrm{ft}$ AMSL & $\mathrm{ft} \mathrm{TOC}^{\mathrm{a}}$ & $\mathrm{ft}$ AMSL & $\mathrm{ft} \mathrm{TOC}^{\mathrm{a}}$ & ft AMSL \\
\hline \multicolumn{10}{|c|}{ Shallow aquifer zone } \\
\hline MW1S & 1351.58 & Dry & - & Dry & - & Dry & - & Dry & - \\
\hline MW11S & 1336.58 & 29.20 & 1307.38 & & & 27.70 & 1308.88 & 24.80 & 1311.78 \\
\hline MW12S & 1327.46 & Dry & - & & & Dry & - & Dry & - \\
\hline \multicolumn{10}{|c|}{ Intermediate aquifer zone } \\
\hline MW10S & 1331.33 & 77.81 & 1253.52 & & & 77.47 & 1253.86 & 73.40 & 1257.93 \\
\hline MW11M & 1336.51 & 82.33 & 1254.18 & & & 82.65 & 1253.86 & 78.85 & 1257.66 \\
\hline MW12M & 1327.46 & 74.50 & 1252.96 & & & 74.77 & 1252.69 & 70.10 & 1257.36 \\
\hline MW13S & 1342.36 & 92.23 & 1250.13 & & & 92.10 & 1250.26 & 87.00 & 1255.36 \\
\hline MW17 & 1351.77 & 102.68 & 1249.09 & & & 101.75 & 1250.02 & 96.60 & 1255.17 \\
\hline \multicolumn{10}{|c|}{ Deep aquifer zone } \\
\hline MW1D & 1351.33 & 124.89 & 1226.44 & 125.54 & 1225.79 & 127.66 & 1223.67 & 119.40 & 1231.93 \\
\hline MW2D & 1348.85 & 122.56 & 1226.29 & 123.20 & 1225.65 & 125.55 & 1223.30 & 117.15 & 1231.70 \\
\hline MW3D & 1345.99 & 126.25 & 1219.74 & 120.09 & 1225.90 & 121.90 & 1224.09 & 113.30 & 1232.69 \\
\hline MW4D & 1326.32 & 101.39 & 1224.93 & 100.54 & 1225.78 & 101.74 & 1224.58 & 93.60 & 1232.72 \\
\hline MW5 & 1327.20 & 102.78 & 1224.42 & & & 102.00 & 1225.20 & 93.80 & 1233.40 \\
\hline MW6S & 1323.13 & 96.10 & 1227.03 & & & 94.50 & 1228.63 & 88.10 & 1235.03 \\
\hline MW6D & 1323.15 & 98.50 & 1224.65 & & & 98.50 & 1224.65 & 89.50 & 1233.65 \\
\hline MW7 & 1329.91 & 105.50 & 1224.41 & 104.48 & 1225.43 & 105.62 & 1224.29 & 97.50 & 1232.41 \\
\hline MW8 & 1330.06 & 105.17 & 1224.89 & & & 104.38 & 1225.68 & 95.75 & 1234.31 \\
\hline MW9 & 1321.86 & 97.20 & 1224.66 & 96.25 & 1225.61 & 96.40 & 1225.46 & 87.65 & 1234.21 \\
\hline MW10D & 1331.33 & 106.22 & 1225.11 & & & 106.36 & 1224.97 & 97.30 & 1234.03 \\
\hline MW11D & 1336.53 & 112.46 & 1224.07 & & & 110.50 & 1226.03 & 102.10 & 1234.43 \\
\hline MW12D & 1327.52 & 102.00 & 1225.52 & & & 103.30 & 1224.22 & 93.70 & 1233.82 \\
\hline MW13D & 1342.37 & 117.83 & 1224.54 & & & 118.19 & 1224.18 & 107.90 & 1234.47 \\
\hline MW14S & 1332.69 & 106.75 & 1225.94 & & & 106.95 & 1225.74 & 99.40 & 1233.29 \\
\hline MW14D & 1332.74 & 107.10 & 1225.64 & 107.23 & 1225.51 & 106.95 & 1225.79 & 101.00 & 1231.74 \\
\hline MW15S & 1309.34 & 84.33 & 1225.01 & & & 84.66 & 1224.68 & 80.30 & 1229.04 \\
\hline MW15D & 1309.29 & 70.20 & 1239.09 & 80.84 & 1228.45 & 80.80 & 1228.49 & 70.30 & 1238.99 \\
\hline MW16S & 1299.47 & 75.30 & 1224.17 & & & 75.50 & 1223.97 & 67.35 & 1232.12 \\
\hline MW16D & 1299.52 & 74.50 & 1225.02 & 74.62 & 1224.90 & 75.00 & 1224.52 & 66.30 & 1233.22 \\
\hline Oentrich ${ }^{b}$ & 1336.93 & $\mathrm{NM}^{\mathrm{C}}$ & & 116.55 & 1220.38 & NM & NM & NM & NM \\
\hline
\end{tabular}

a TOC, top of casing.

b The Oentrich well water level was measured from the concrete at the top of the well vault. The value shown was corrected by $5.5 \mathrm{ft}$ to give a measured depth from the top of the casing.

c NM, not measured (sampled from faucet). 


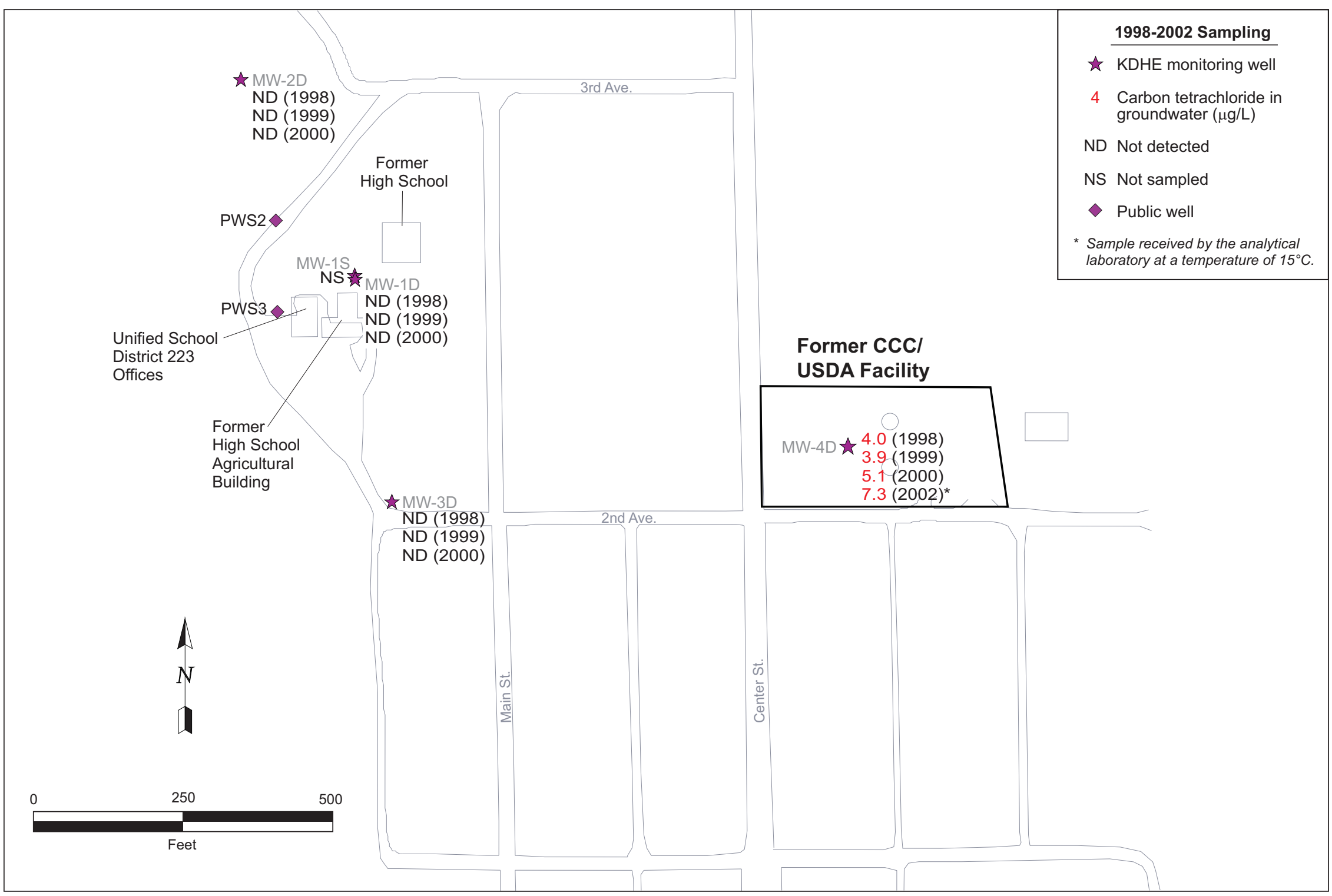

FIGURE 3.1 Carbon tetrachloride concentrations detected in groundwater in the KDHE's 1999 comprehensive investigation and subsequent sampling. Source of data: BE\&K (1999, 2000); KDHE (2002). 


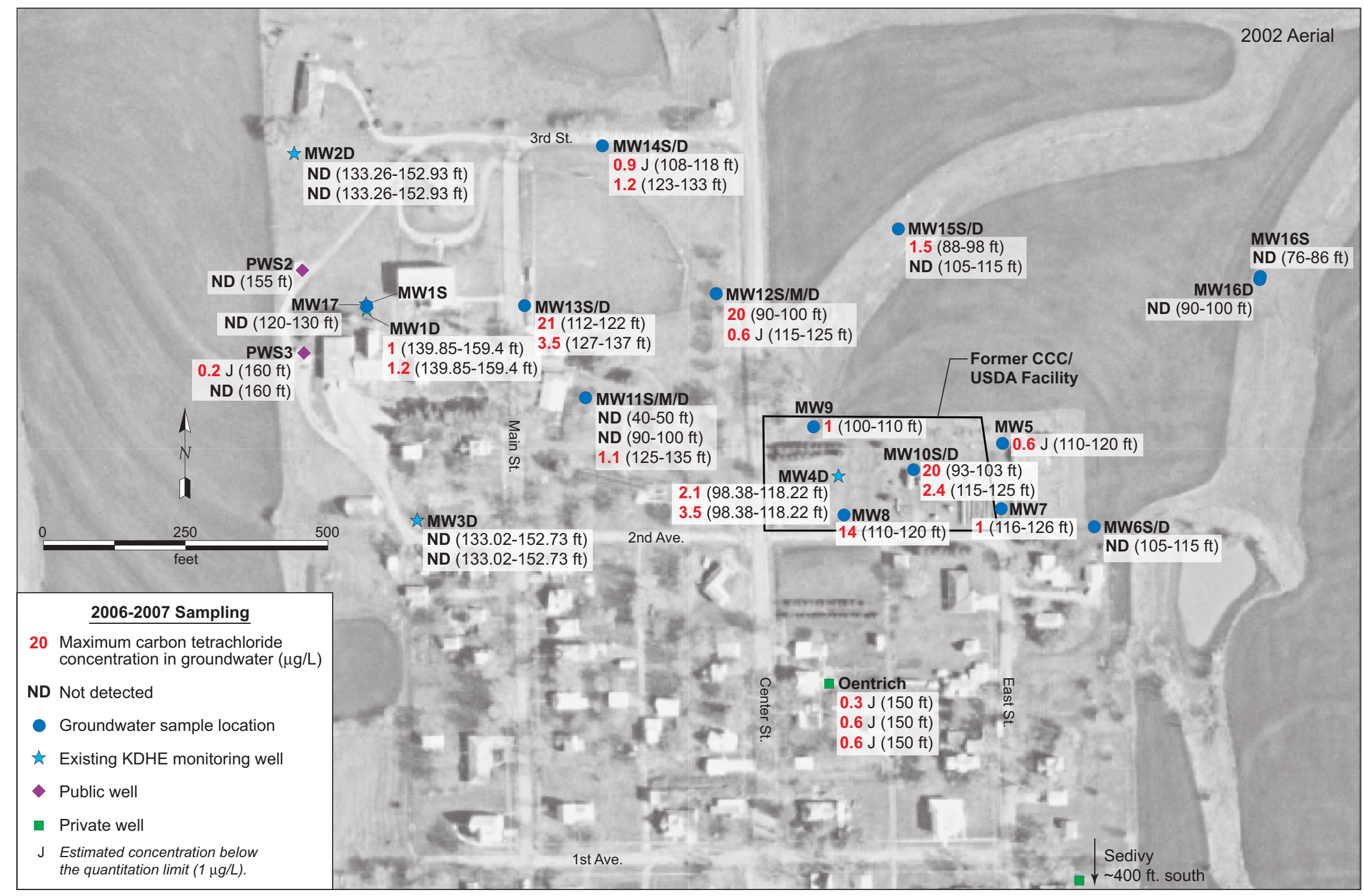

FIGURE 3.2 Analytical results for carbon tetrachloride in groundwater samples collected during the 2006-2007 CCC/USDA-Argonne investigation at Barnes. Source of photograph: NAPP (2002). 


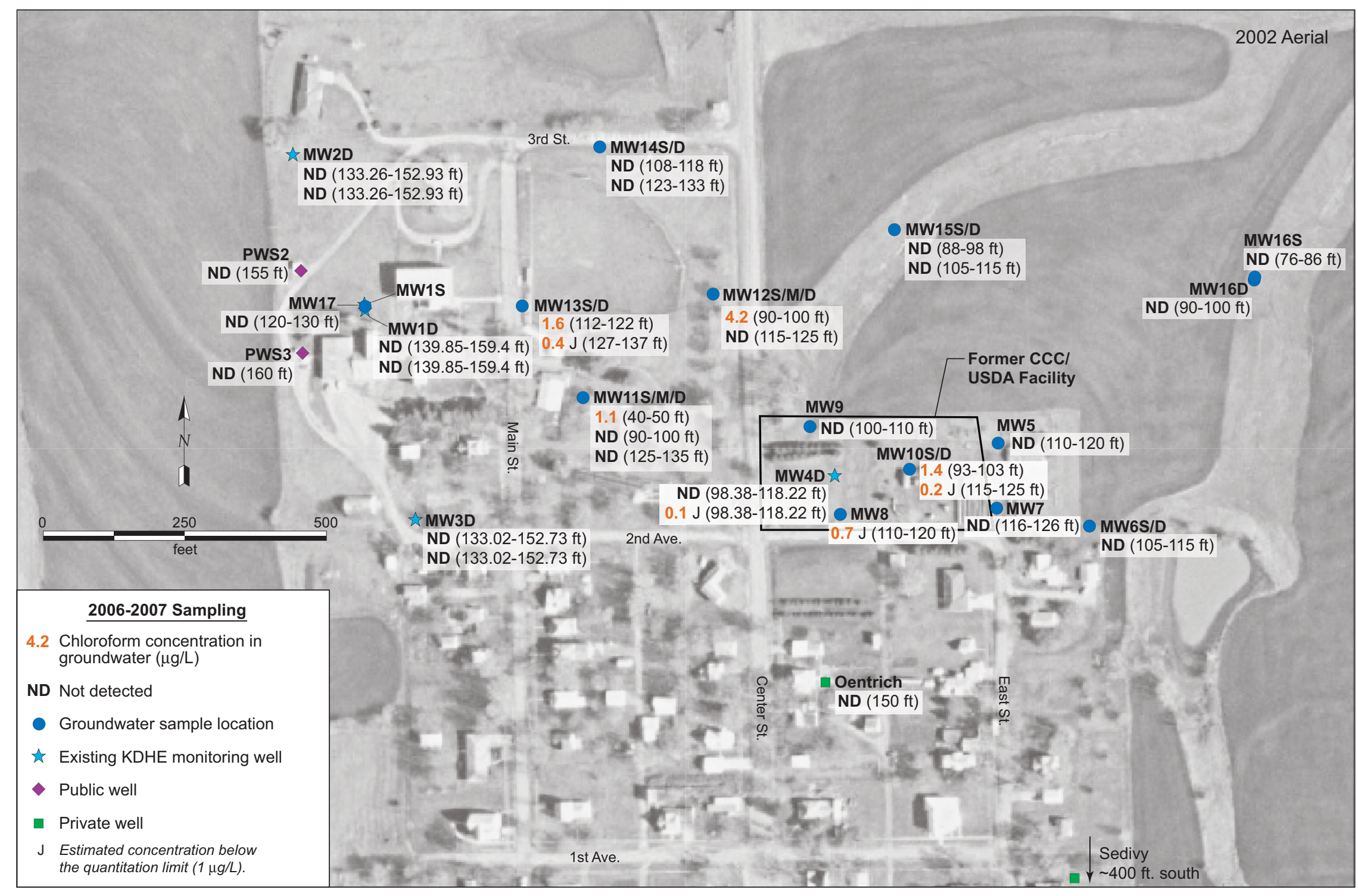

FIGURE 3.3 Analytical results for chloroform in groundwater samples collected during the 2006-2007 CCC/USDA-Argonne investigation at Barnes. Source of photograph: NAPP (2002). 


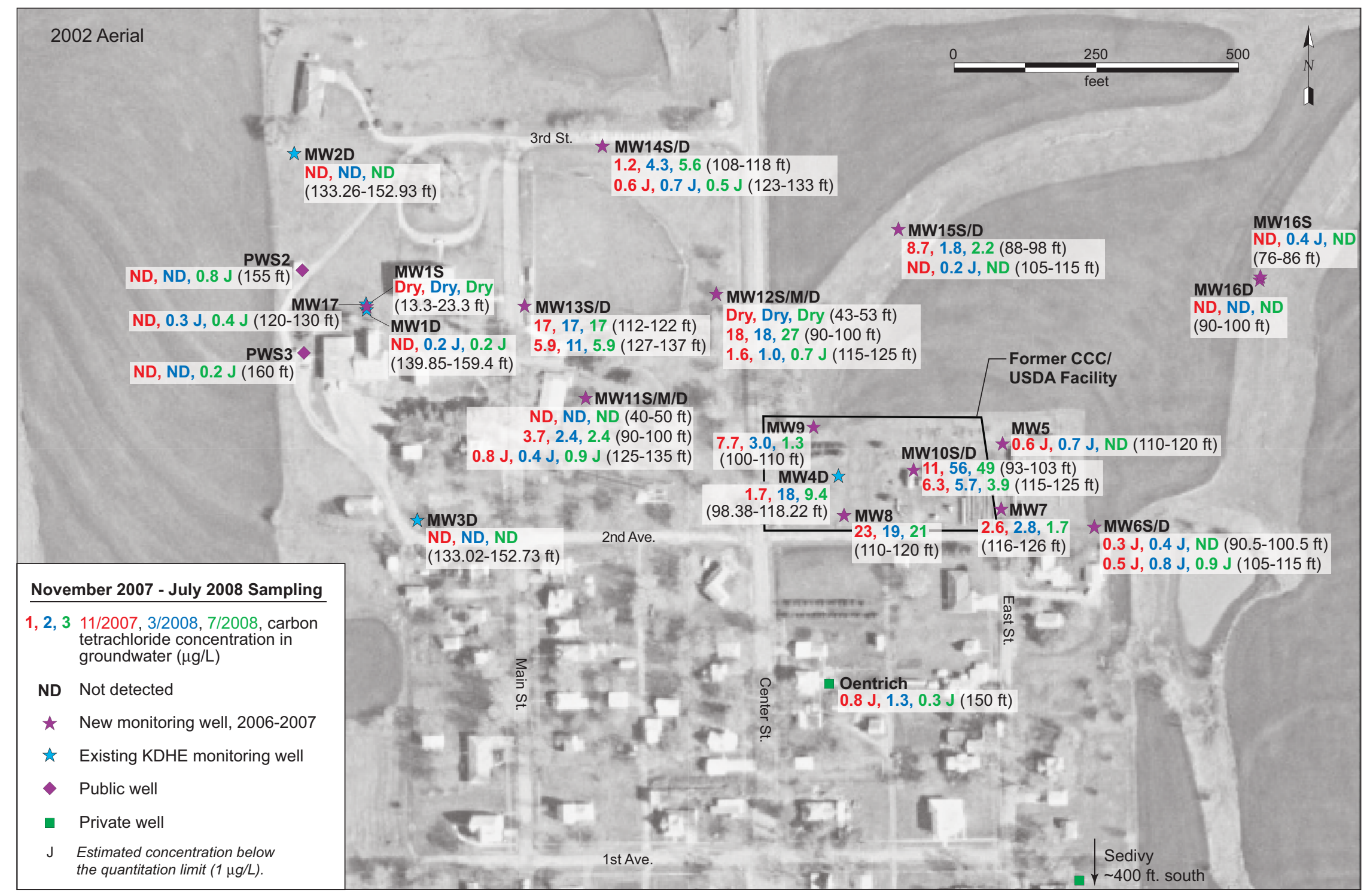

FIGURE 3.4 Analytical results for carbon tetrachloride in groundwater samples collected during CCC/USDA-Argonne monitoring in November 2007-July 2008 at Barnes. Source of photograph: NAPP (2002). 


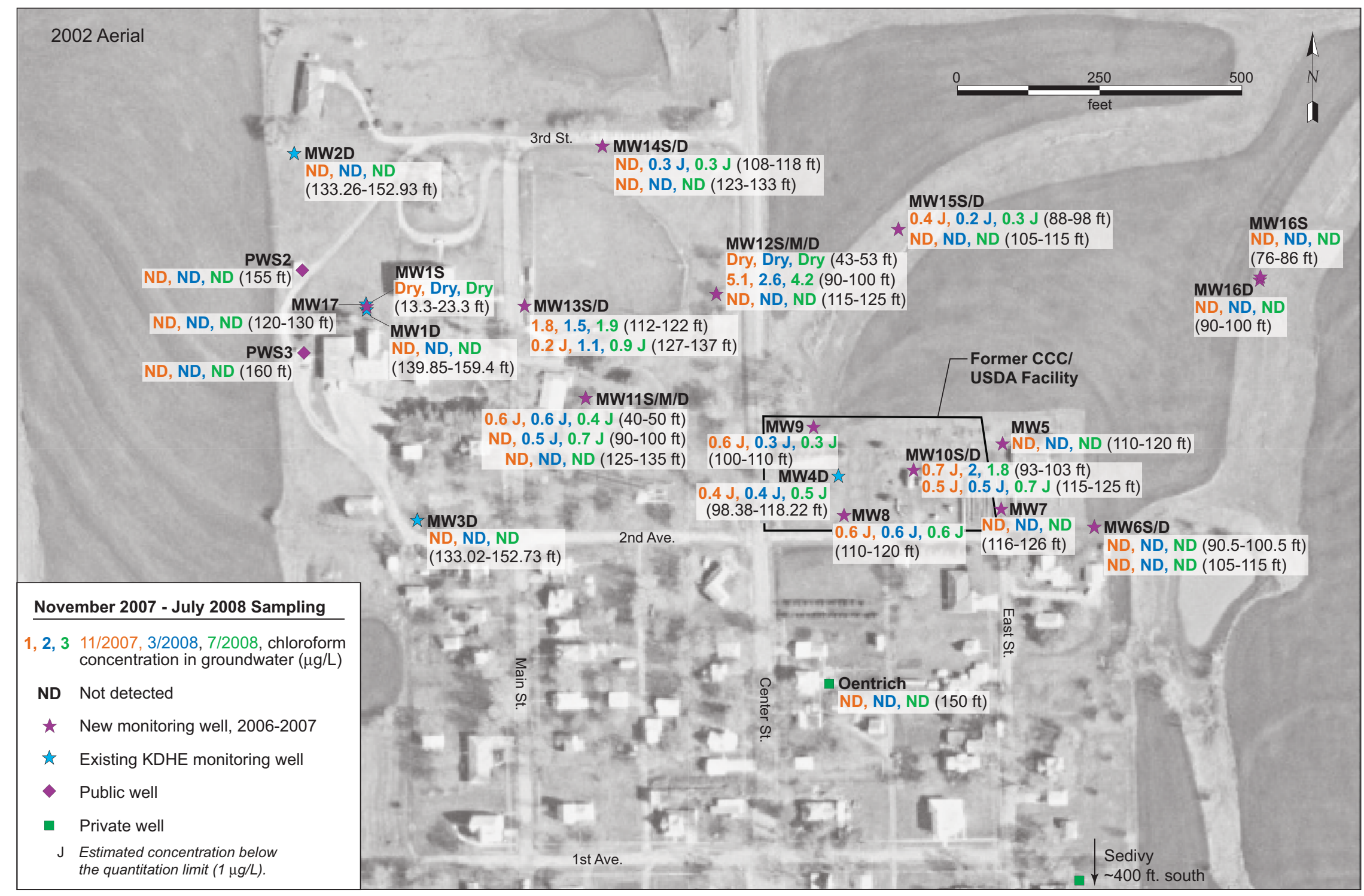

FIGURE 3.5 Analytical results for chloroform in groundwater samples collected during CCC/USDA-Argonne monitoring in November 2007-July 2008 at Barnes. Source of photograph: NAPP (2002). 


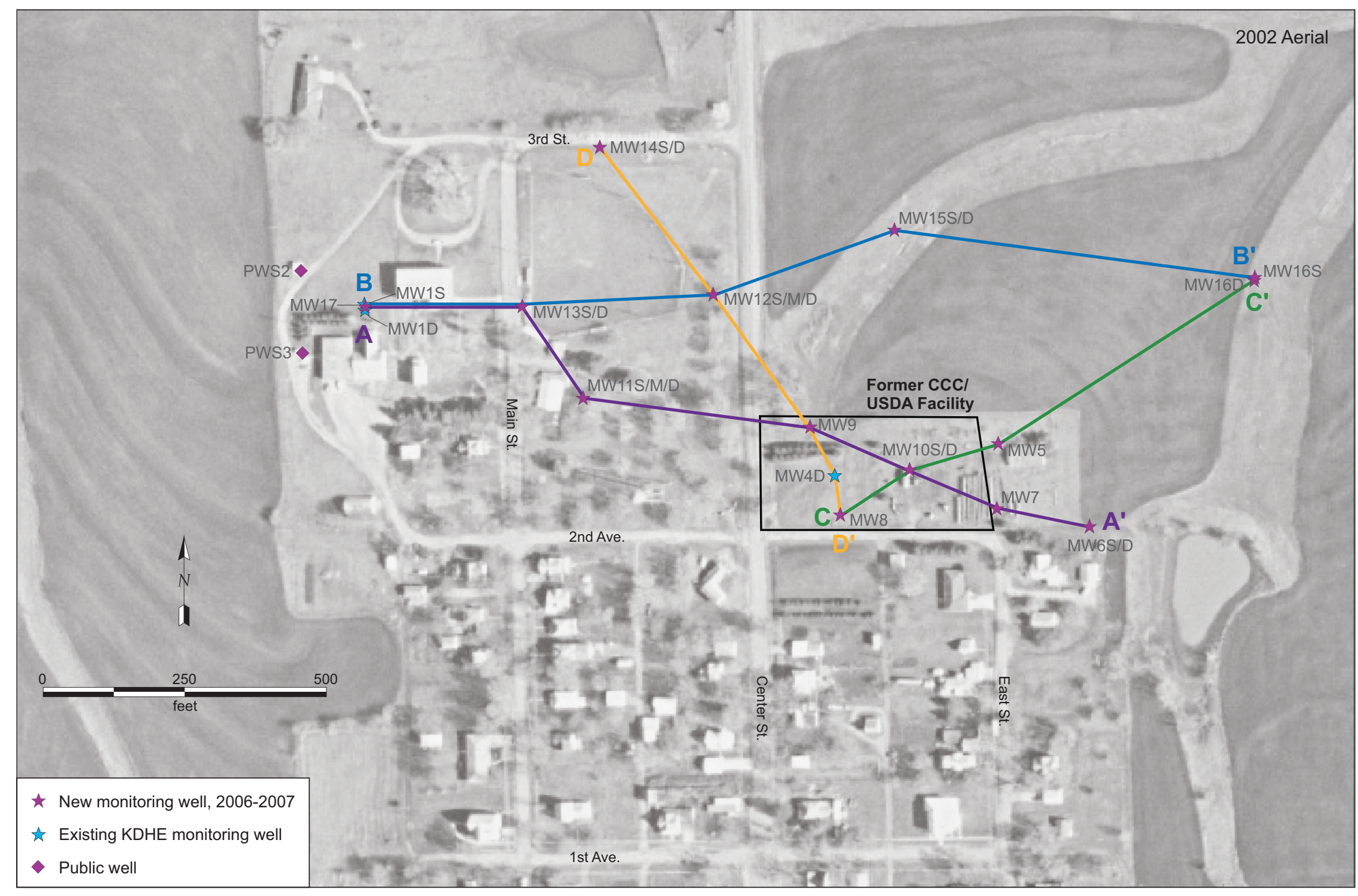

FIGURE 3.6 Locations of interpretive hydrogeologic cross sections. Source of photograph: NAPP (2002). 


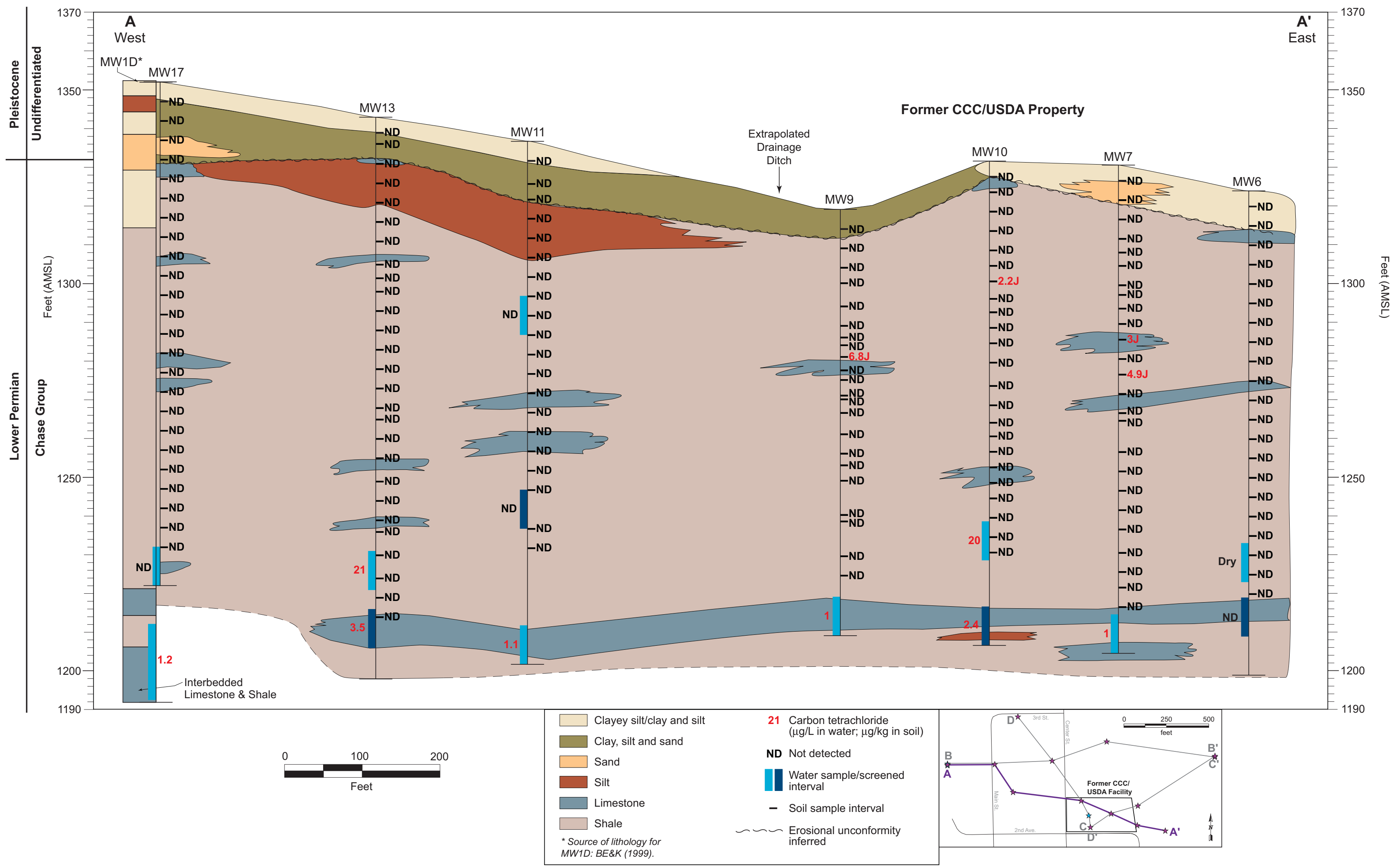

FIGURE 3.7 West-east interpretive hydrogeologic cross section A-A' across the former CCC/USDA grain storage property at Barnes (vertically exaggerated), illustrating the lateral and vertical distribution of carbon tetrachloride in soil and groundwater in 2006-2007. 


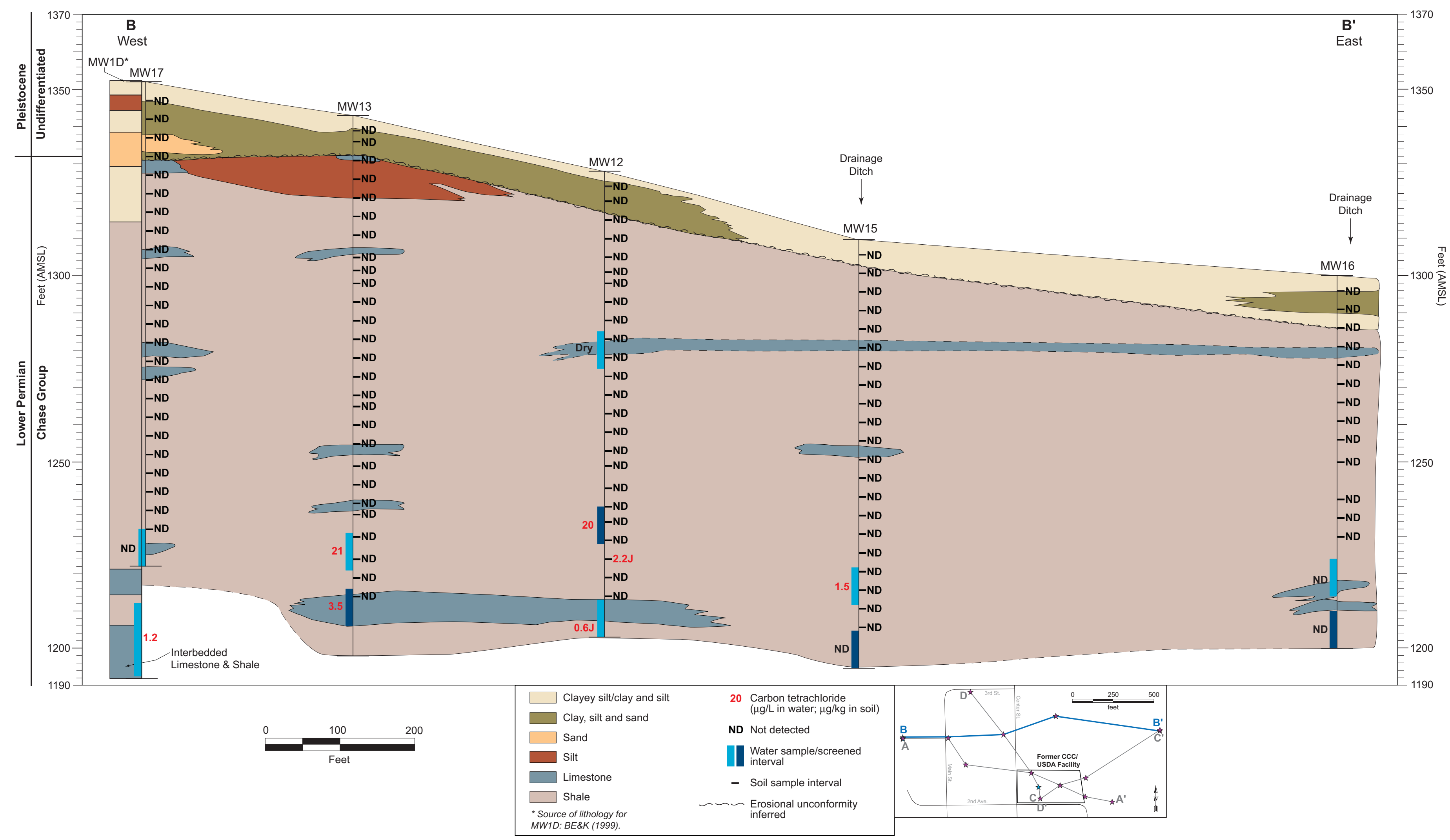




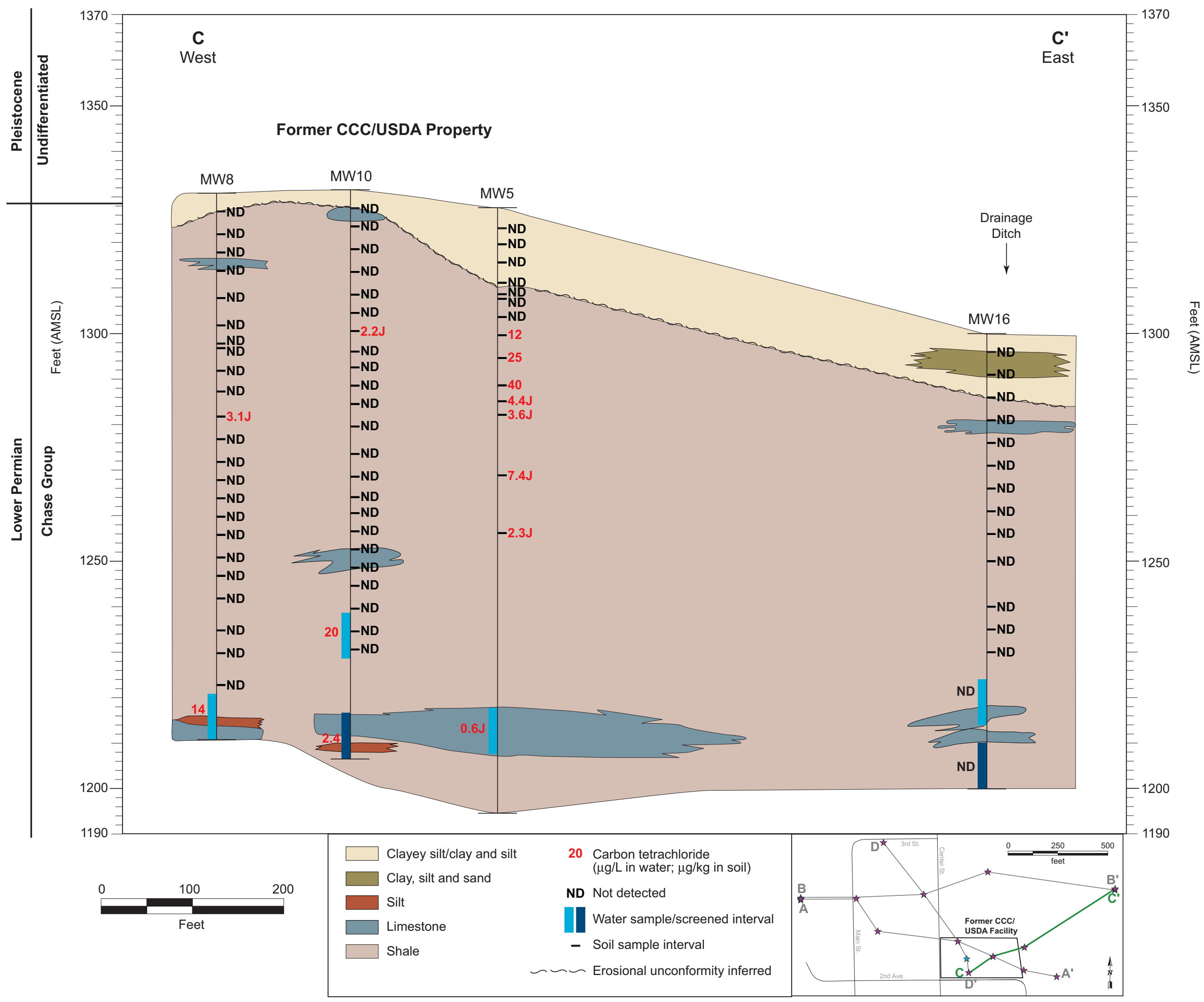

FIGURE 3.9 West-east interpretive hydrogeologic cross section C-C' across the former CCC/USDA grain storage property at Barnes (vertically exaggerated) illustrating the lateral and vertical distribution of carbon tetrachloride in soil and groundwater in 2006-2007. 


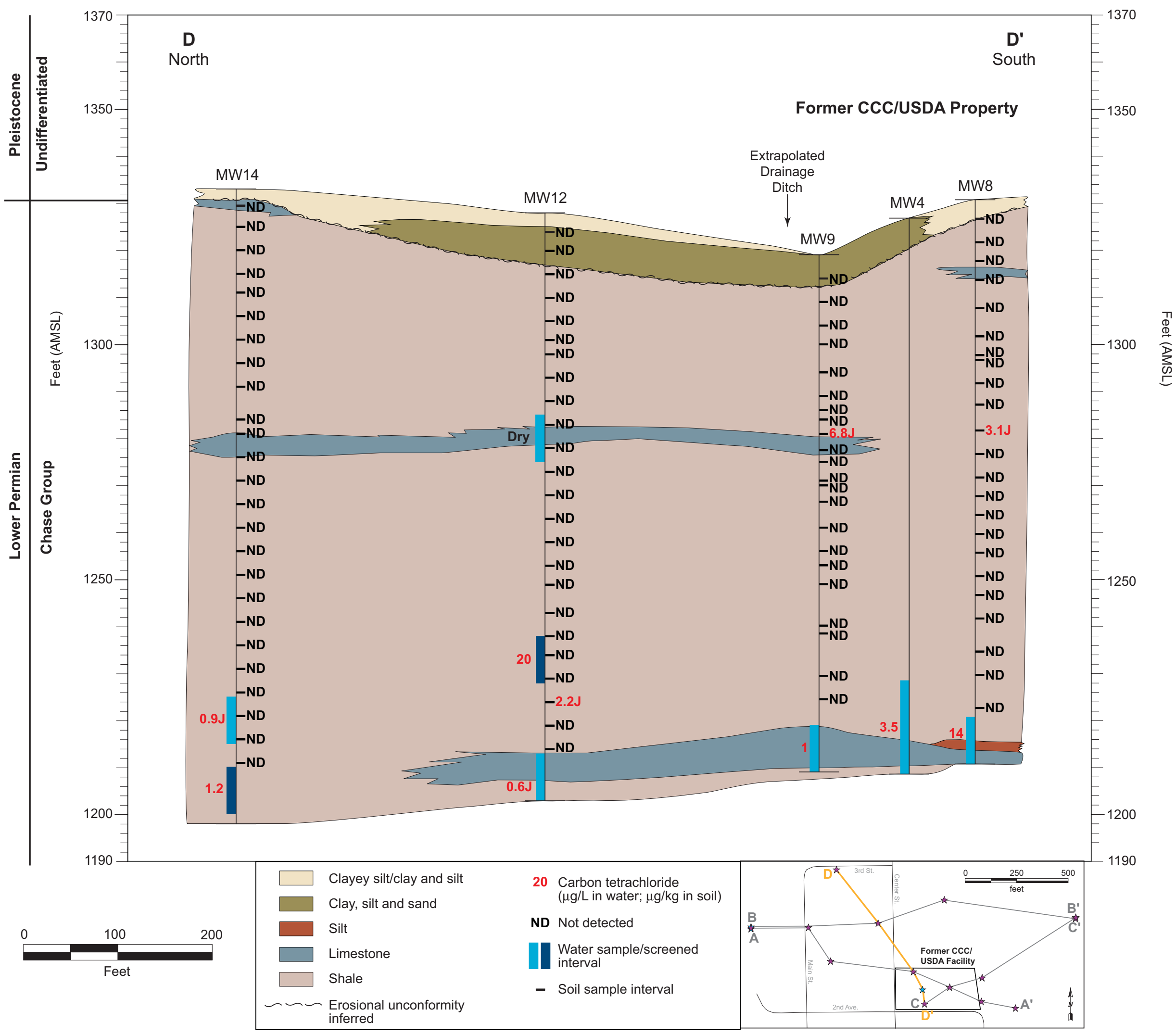

FIGURE 3.10 North-south interpretive hydrogeologic cross section D-D' across the former CCC/USDA grain storage property at Barnes (vertically exaggerated), illustrating the lateral and vertical distribution of carbon tetrachloride in soil and groundwater in 2006-2007. 


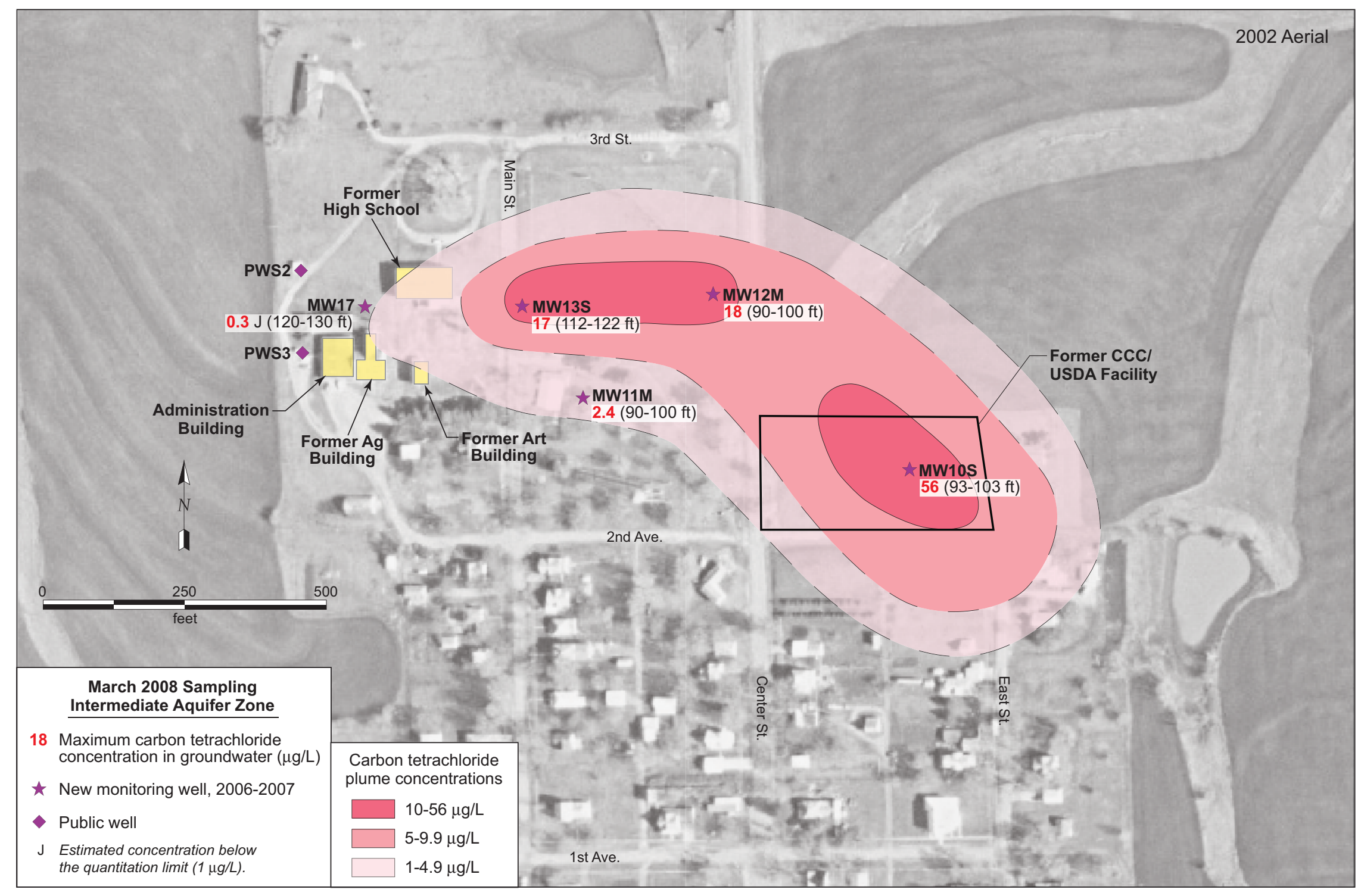

FIGURE 3.11 Interpreted carbon tetrachloride plume in wells screened in the intermediate aquifer (roughly 1,255 ft AMSL) in March and July 2008. Source of photograph: NAPP (2002). 


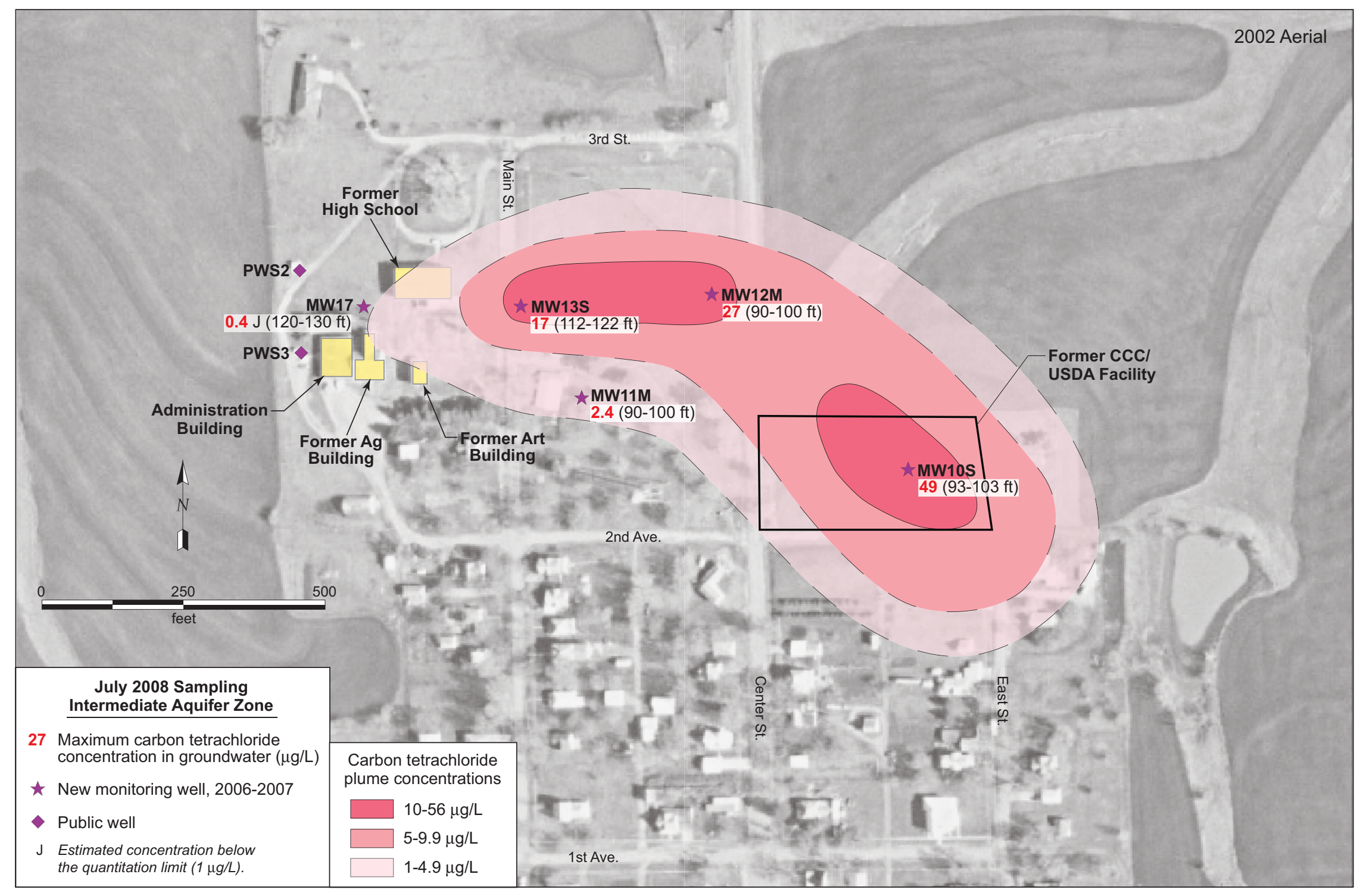

FIGURE 3.11 (cont.) 


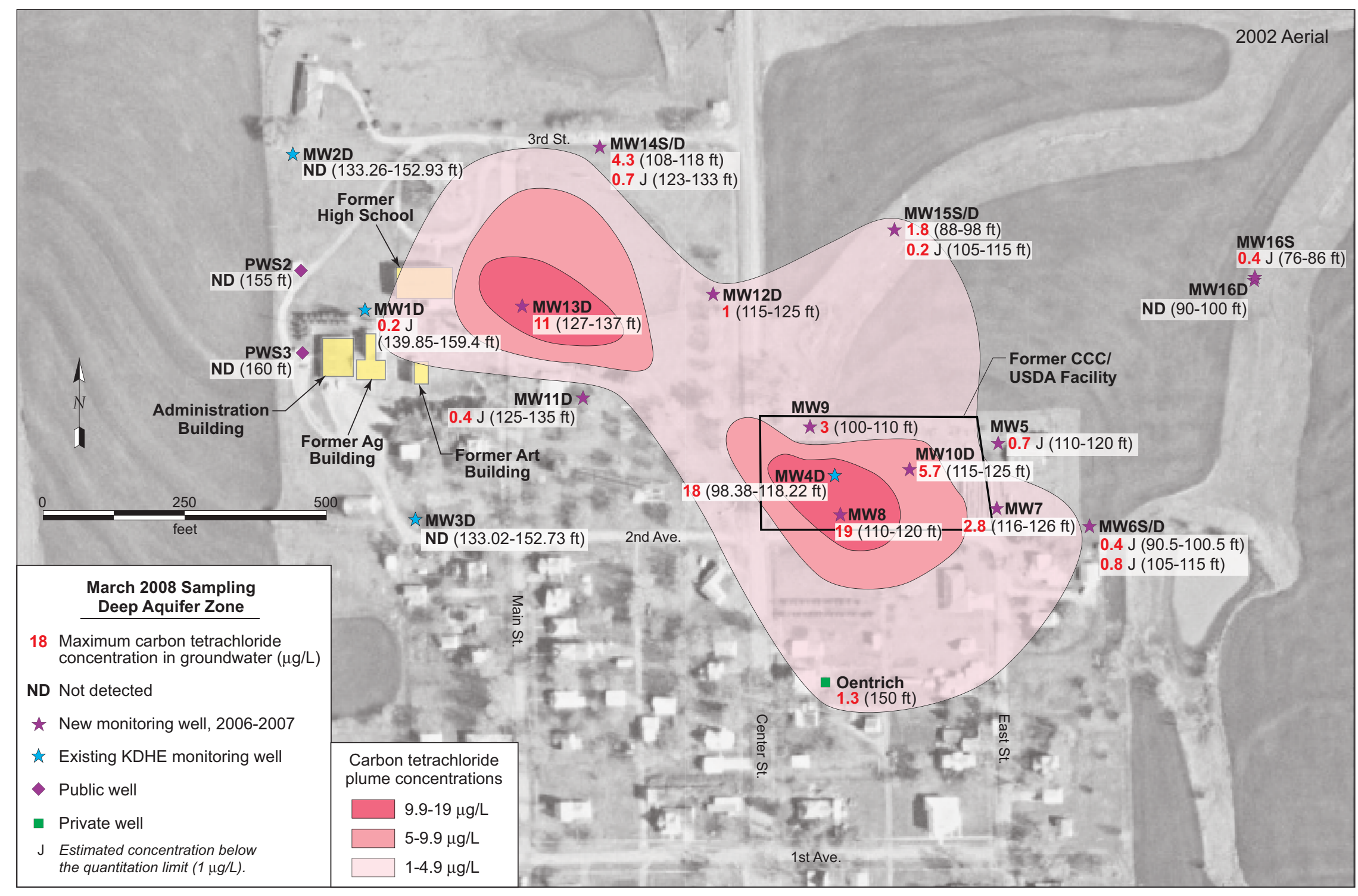

FIGURE 3.12 Interpreted carbon tetrachloride plume in wells screened in the deep aquifer (roughly 1,230 ft AMSL) in March and July 2008. Source of photograph: NAPP (2002). 


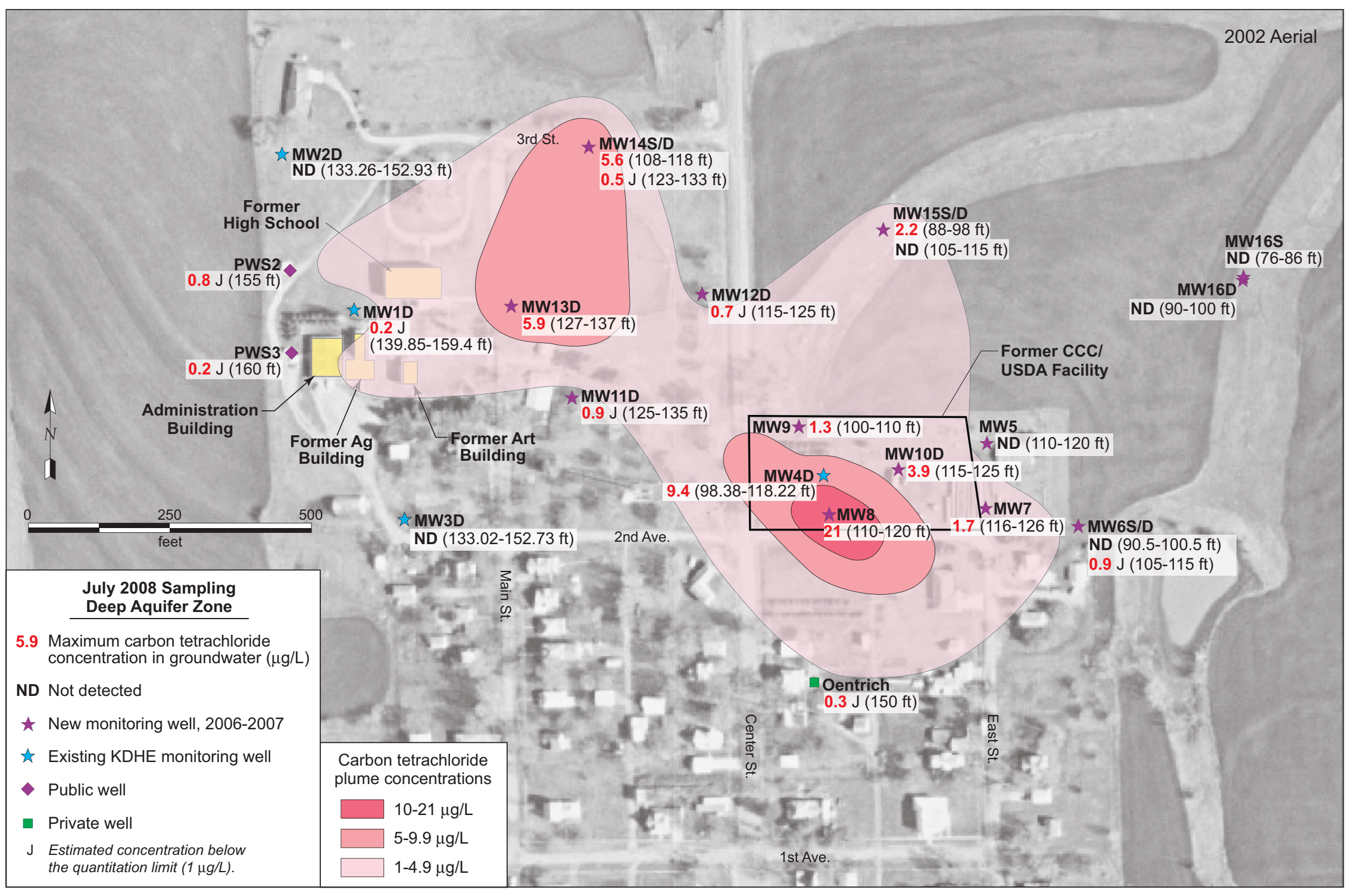

FIGURE 3.12 (Cont.) 
Barnes Monitoring Data

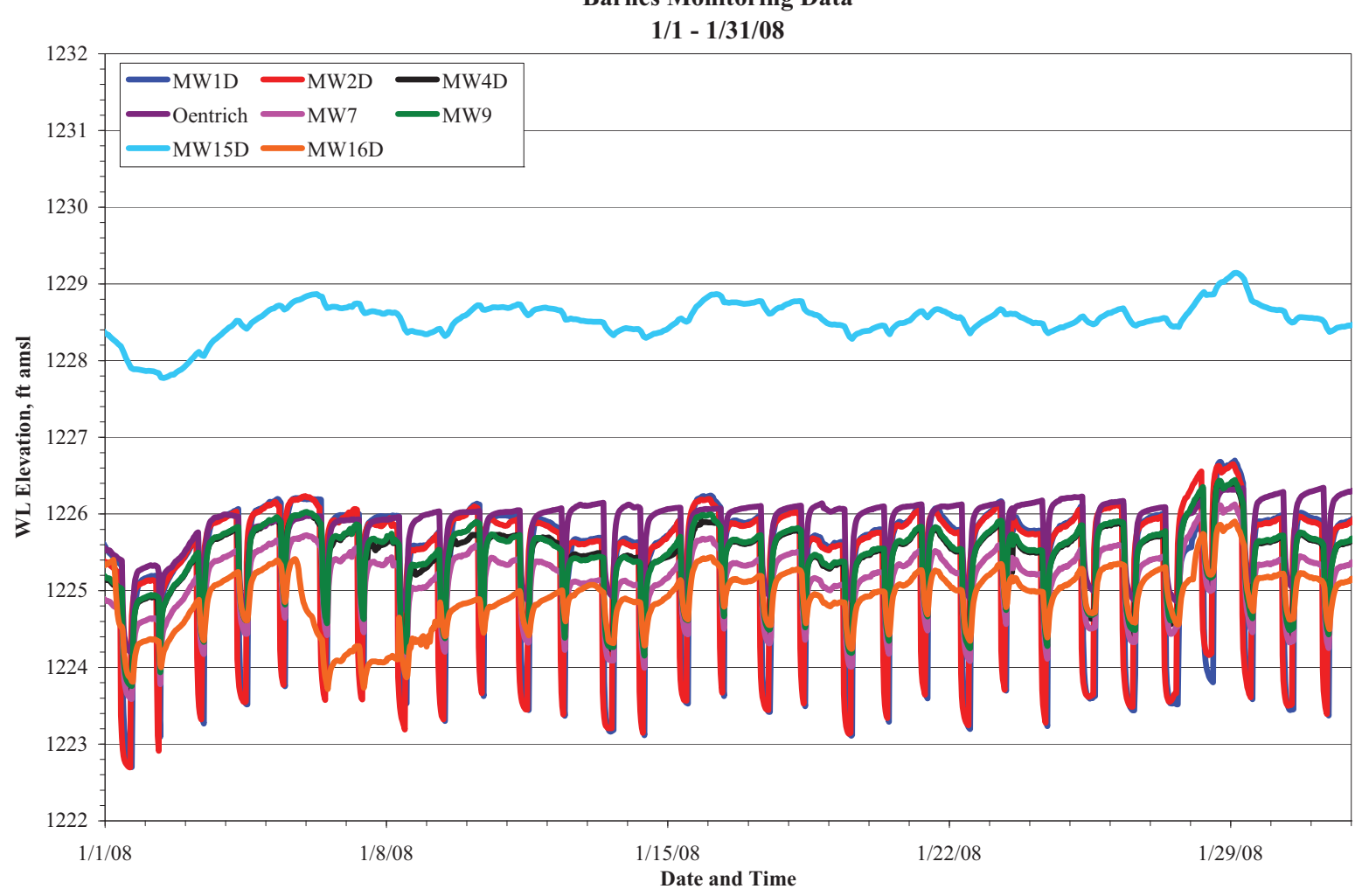

Barnes Monitoring Data

$2 / 1-2 / 29 / 08$

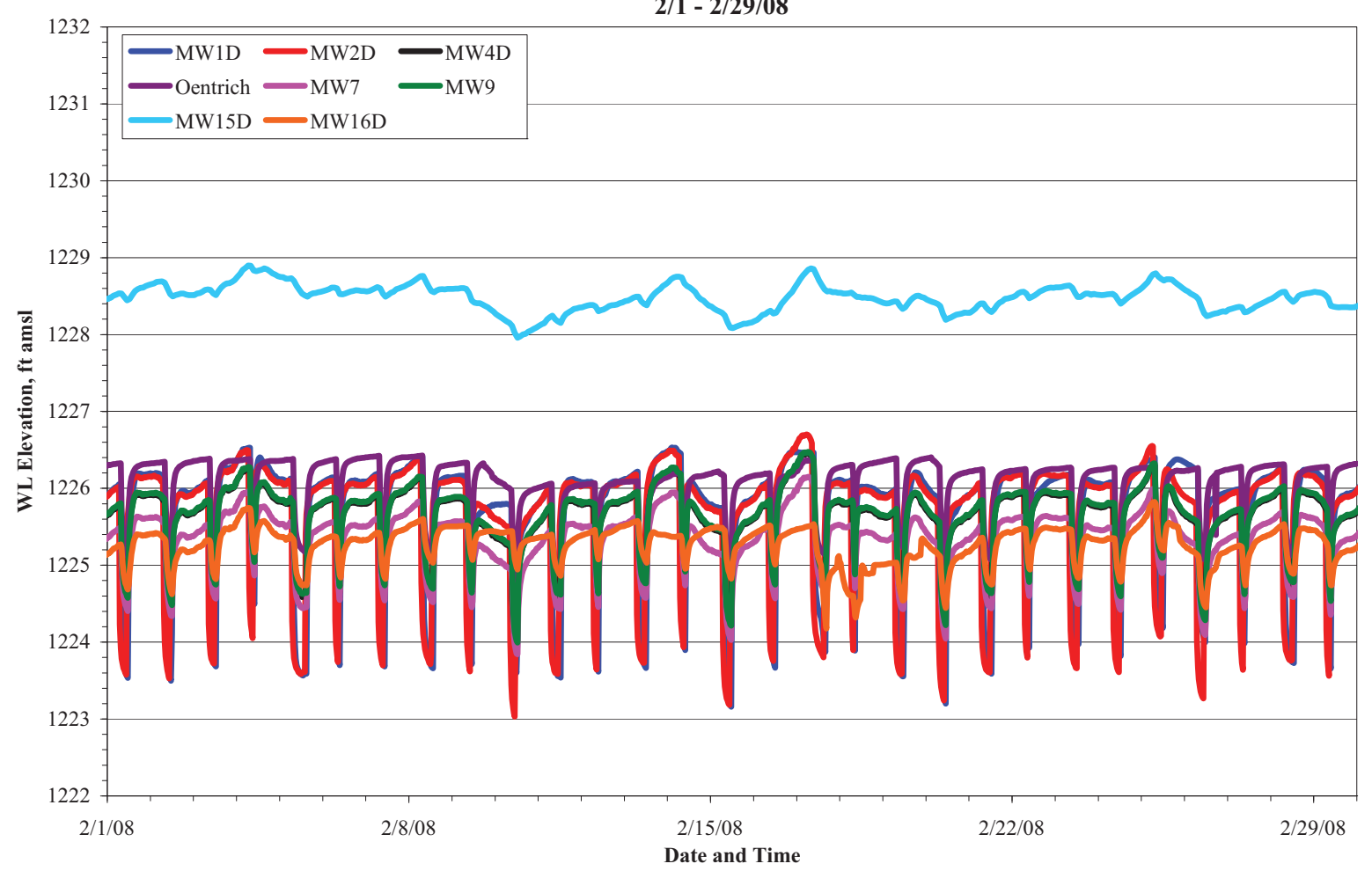

FIGURE 3.13 Hydrographs summarizing monthly results of long-term water level monitoring in wells at Barnes in January-June 2008. 

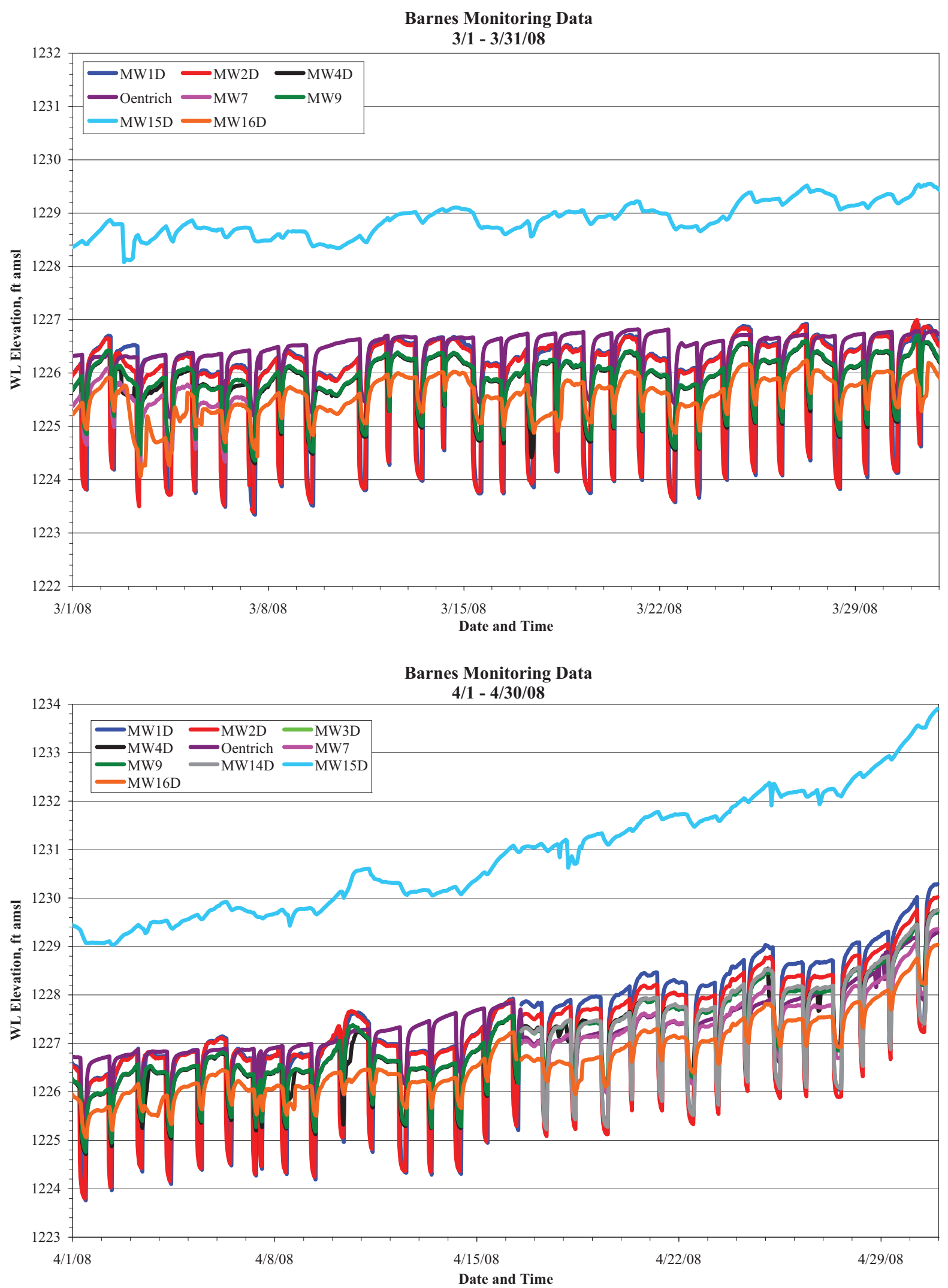

FIGURE 3.13 (Cont.) 

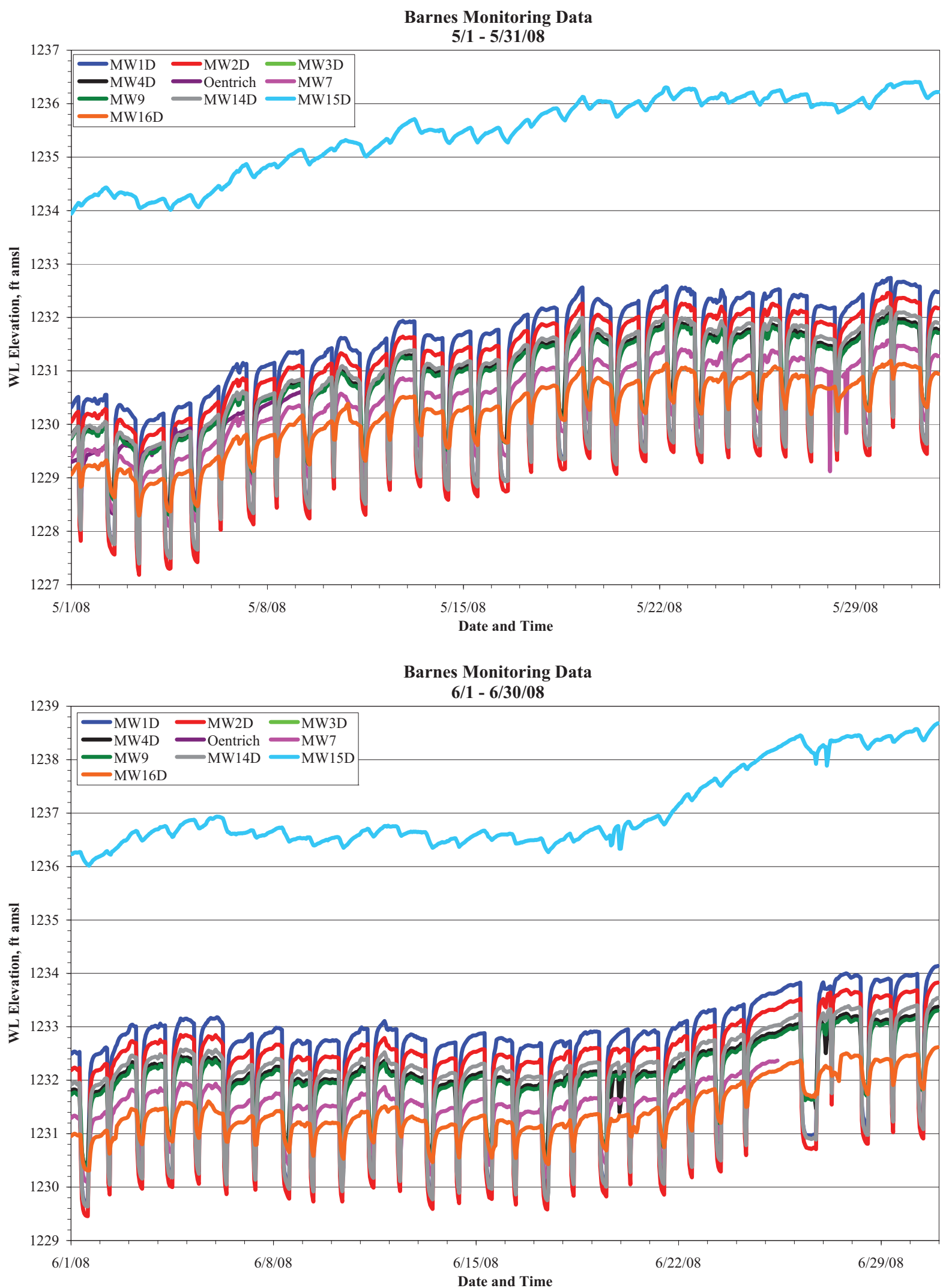

FIGURE 3.13 (Cont.) 

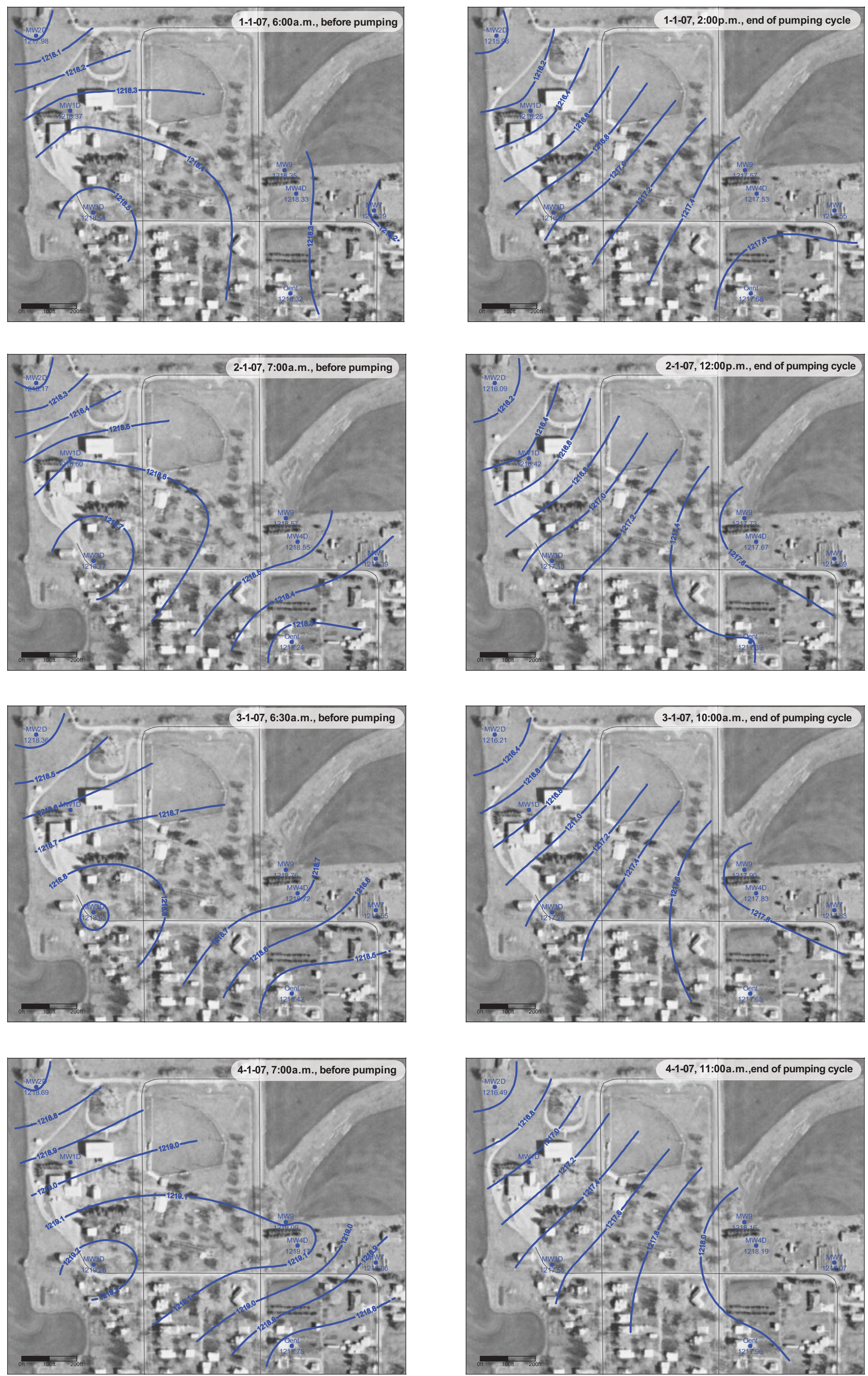

FIGURE 3.14 Potentiometric surface maps depicting the groundwater flow direction at Barnes in January-April 2007, before pumping of the public water supply wells (left panels) and during pumping (right panels). Source of photograph: NAPP (2002). 

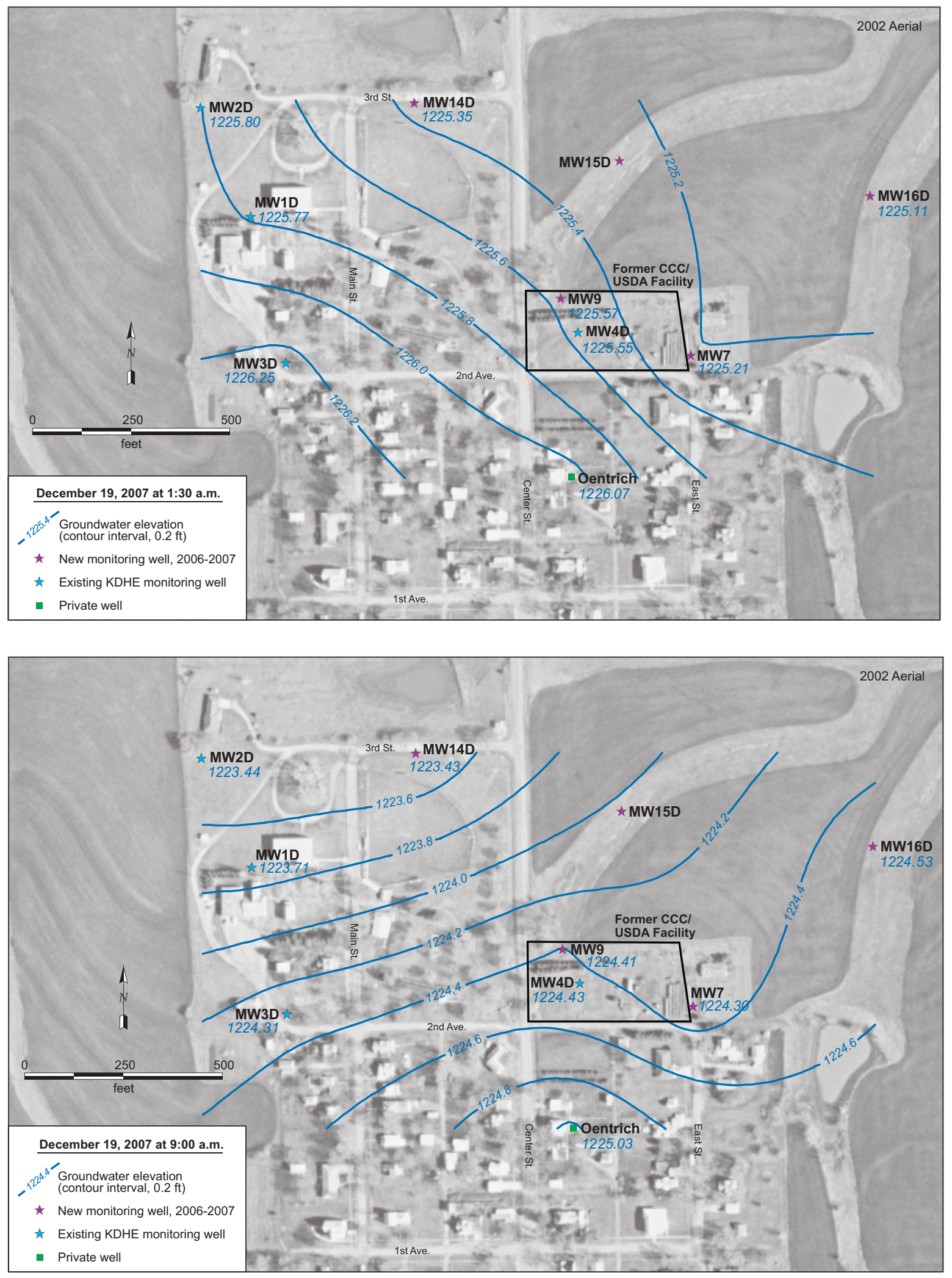

FIGURE 3.15 Potentiometric surface maps depicting the groundwater flow direction at Barnes in December 2007 and May 2008, before pumping of the public water supply wells (top panels) and during pumping (bottom panels). Source of photograph: NAPP (2002). 

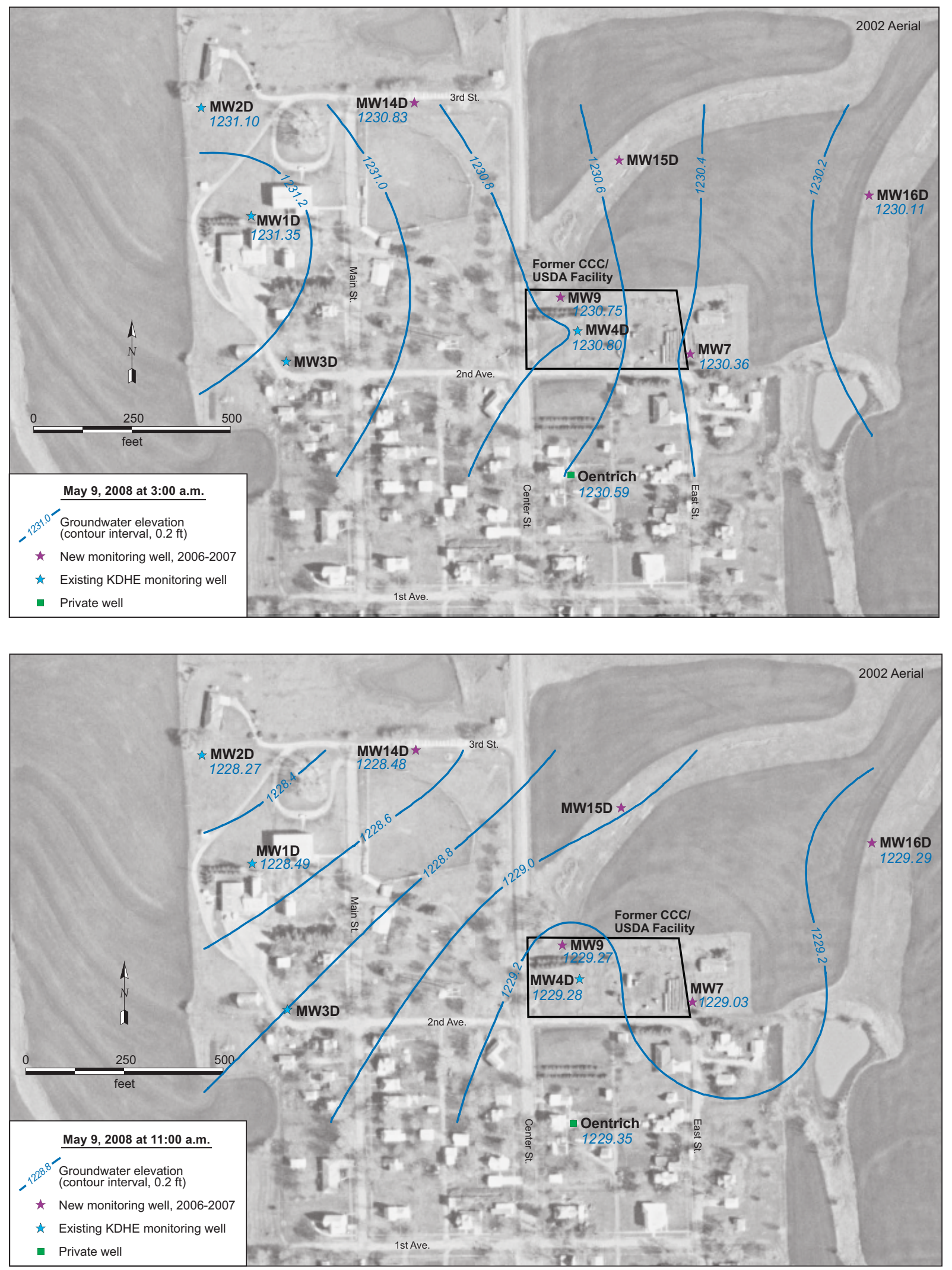

FIGURE 3.15 (Cont.) 


\section{Proposed Interim Measure}

\subsection{Interim Measure Evaluation}

In response to the KDHE's request (KDHE 2008a) for the development of a contingency IM for implementation of a wellhead treatment system at PWS2 and PWS3, the CCC/USDA requested that Argonne investigate and evaluate the best technical options for addressing the possible treatment of the groundwater at these wells. In the course of this investigation, three potential options were evaluated in depth. These include the following:

1. Replacement of the current water supply through connection to Washington County RWD \#2.

2. Granular activated carbon (GAC)

3. Air stripping

\subsubsection{Option 1 - Replacement of Current Water Supply}

The option of providing water from Washington County RWD 2 was considered, and discussions were held with the water district manager. This option was not selected for the following reasons:

- The estimated cost associated with laying lines over the 3.5-mi distance necessary to connect the approximately 104 Barnes residences to the RWD.

- The RWD's lack of experience in supplying water to an entire town and the general reservations expressed by the water district representatives in that regard.

- The potential requirement to upgrade the entire RWD infrastructure to handle the considerable increase in demand for supplied water to the citizens of Barnes. 


\subsubsection{Options 2 and 3 - GAC and Air Stripping}

The factors evaluated for both GAC and air stripping were

- The technical applicability at this site;

- The present general water quality of the PWS system;

- The potential future impact to the general water quality;

- The cost of installing each system; and

- The operation, maintenance, and monitoring (OMM) costs for each system.

Evaluation of the two systems indicates the following:

1. The applicability of GAC treatment and air stripping technologies for use at the Barnes public water supply wells appears to be similar. Both treatment methods can be engineered to address the concentrations of carbon tetrachloride and chloroform that have been detected at this site.

2. Preliminary testing of the inorganic and bacteriological content of both public water supply wells indicated that neither technology would be significantly more beneficial than the other to implement.

3. The potential impact to the general water quality might be a factor in the use of air stripping. Whereas air stripping technology might alter the aesthetic quality of the water, including the taste, a GAC system would be less likely to affect the general water quality and taste.

4. The costs for installation and OMM of each system suggest that the GAC technology would be slightly more expensive to install than air stripping; however, the long-term OMM costs for the two systems are comparable. 
5. In terms of OMM, both systems will require some oversight to ensure optimal performance. GAC will require periodic carbon changes and backwashing. Air stripping will require periodic tray cleaning.

6. Both systems can be fitted with advanced telemetry to monitor system performance.

The preferred contingency IM technology proposed for implementation at the Barnes public water supply wells is installation of a GAC unit.

Installation of the GAC treatment system proposed as the preferred remedial technology will accomplish the following:

- Treat contaminated groundwater at the PWS2 and PWS3 wellheads.

- Maintain carbon tetrachloride concentrations below the MCL and Tier 2 RBSL standard of $5.0 \mu \mathrm{g} / \mathrm{L}$.

Upon approval of this contingency IM conceptual design, the CCC/USDA intends to complete the following tasks:

- Develop a treatment system design document for KDHE review and approval.

- Identify the necessary permits from the appropriate KDHE divisions for activation when the KDHE and the CCC/USDA determine that full implementation of the contingency IM is required for the protection of public health.

\subsection{Interim Measure Implementation}

It is proposed that implementation of the proposed methodology be initiated in accordance with Kansas regulations and policies regarding public water supply systems — in the case of carbon tetrachloride, if a Tier 2 violation occurs. The applicable regulations, which are defined in K.A.R. 28-15, include the following requirements: 
- Quarterly sampling is mandated after a detection of the contaminant of concern at $0.5 \mu \mathrm{g} / \mathrm{L}(0.0005 \mathrm{mg} / \mathrm{L}$, one-tenth of the MCL).

- If, after a period of quarterly sampling, (1) the results demonstrate that the contaminant level is reliably and consistently below the MCL (though the contaminant is still present) and (2) the minimum number of quarterly samples have been collected (four sampling events), then the quarterly sampling requirement can be decreased to annual sampling.

- The compliance sampling points for wells PWS2 and PWS3 will be the respective wellheads. Periodically, on the established monitoring schedule, the CCC/USDA will continue to collect untreated or "raw" water samples from the individual wellheads prior to treatment. In the event that the carbon tetrachloride and/or chloroform concentration in the untreated water at the wellhead is at a level to constitute a Tier 2 violation (i.e., above the MCL), the CCC/USDA will collect a confirmation sample within 30 days of the original sample. If elevated levels of these compounds in groundwater are confirmed and persist in a running annual average, or as otherwise established by CFR 141.24 (f)(15), the CCC/USDA will initiate the development of the detailed interim measure engineering design and will submit the design plan for KDHE review within 45 days, as defined in K.A.R. 28-15.

- Implementation of the contingency IM will be initiated upon receipt of all required KDHE approvals. 


\section{Interim Treatment System Objectives}

The objectives of the contingency IM proposed for the Barnes public water supply system are as follows:

- Have an approved treatment system design in place for immediate implementation, should that become necessary.

- Provide for treatment of the contaminated groundwater at the PWS2 and PWS3 wellheads, as necessary, to maintain carbon tetrachloride concentrations in the treated effluent below the current MCL and KDHE Tier 2 RBSL values of $5.0 \mu \mathrm{g} / \mathrm{L}$.

- $\quad$ Provide an uninterrupted supply of water for domestic use. 


\section{Interim Measure Design}

\subsection{Design Basis}

The remedial system considered for the Barnes IM involves the installation of a GAC treatment system at the public water supply wellheads. Specifically, the proposed system includes installation of vessels containing granular activated carbon. Groundwater from the public supply will be pumped through the vessels to accomplish removal of residual VOCs (including chlorinated compounds). The treated water will then be pumped through the city's chlorination treatment system and into the city's distribution system.

This type of system has been used successfully for at least 13 public water supply systems throughout Kansas (Table 6.1). The implementation of GAC systems allowed the affected cities to continue using their water for distribution as a drinking water supply. The advantage of using GAC vessels includes the ease of installation and the minimal maintenance required for optimal system performance.

\subsection{Design Specifications}

The proposed remedial system for this contingency IM conceptual design includes four vessels, each containing 2,000 lb of virgin GAC. The vessels will be housed in a building that is separate from but adjacent to the public wells. Two of the vessels will be used for the primary treatment of contaminanted water. The other two vessels will be used for polishing and as backup units. Placement of the building and vessels will need to be discussed with and authorized by the city of Barnes. Detailed information will be included in the final treatment system design document.

Although the design of the proposed system has not been finalized pending KDHE approval of the contingency IM conceptual design, the preliminary design parameters indicate that the proposed system will remove carbon tetrachloride successfully at concentrations up to $200 \mu \mathrm{g} / \mathrm{L}$. An estimated $60-80 \mathrm{lb}$ of carbon will be spent per quarter. Prior to completion of the final design, the groundwater will need to be tested for the content of inorganic materials. Furthermore, the system design will include the most efficient mechanism for handling the wastewater that will be generated as part of the backwashing process. Backwashing is needed 
periodically to redistribute the carbon in the vessels and eliminate preferential pathways that can be created by continual flow through the system. Approximately 1,050 gal of wastewater per vessel will be generated for each backwash event. This water will need to be containerized and tested prior to discharge. Details regarding the backwashing procedure and handling of wastewater will be included in the final design.

TABLE 6.1 Public water supply systems in Kansas where granular activated carbon vessels have been or are currently being used to treat groundwater contaminated with volatile organic compounds (KDHE 2008b).

\begin{tabular}{|c|c|c|c|c|}
\hline City & $\begin{array}{l}2007 \\
\text { Population }\end{array}$ & $\begin{array}{l}\text { Vessel } \\
\text { Size (lb) }\end{array}$ & $\begin{array}{l}\text { Number of } \\
\text { Vessels }\end{array}$ & $\begin{array}{l}\text { GAC System } \\
\text { Use Status }\end{array}$ \\
\hline Atwood & 1,092 & 10,000 & 2 & In active use \\
\hline Ellis & 1,913 & 2,000 & 4 & In active use \\
\hline Hanston & 245 & 20,000 & 2 & In active use \\
\hline Manhattan & 51,707 & 20,000 & 2 & In active use \\
\hline Manter & 162 & 10,000 & 2 & In active use \\
\hline Miltonvale & 467 & 10,000 & 2 & In active use \\
\hline Moscow & 233 & $10,000 \mathrm{~s}$ & 4 & In active use; 2 wells \\
\hline Oakley & 1,870 & 10,000 & 2 & $\begin{array}{l}\text { Source area remediated; } \\
\text { system in standby mode }\end{array}$ \\
\hline Park & 131 & 2,000 & 4 & In active use \\
\hline Park City & 7,588 & 20,000 & 2 & In active use \\
\hline Quinter & 809 & 2,000 & 4 & $\begin{array}{l}\text { Source area remediated; } \\
\text { system in standby mode }\end{array}$ \\
\hline Satanta & 1,124 & 10,000 & 2 & In active use \\
\hline Scott City & 3,494 & $\begin{array}{l}10,000 \\
30,000\end{array}$ & $\begin{array}{l}2 \\
2\end{array}$ & In active use; 2 wells \\
\hline
\end{tabular}


Upon KDHE approval of this contingency IM conceptual design, an experienced contractor with proven knowledge in the design and operation of GAC systems will be procurred. A final design will be developed and submitted to the KDHE for review and approval. Consistent with the guidance in BER Policy \#BER-RS-029 (KDHE 2006a), the following will be included in the final design document:

- Justification for and benefits of the IM

- Design layout of each carbon vessel

- Design layout of the building

- Location layout

- $\quad$ Permit requirements

- Relevant illustrations

- Detailed working schedule (generalized at the time of this submittal)

- Detailed operations and maintenance plan

- $\quad$ Proposed performance monitoring plan

\subsection{Drawings/Schematics}

A specific design drawing for the Barnes system will be developed for the treatment system design document.

\subsection{Cost Estimate}

Recent discussions with experienced contractors indicate that the current cost of a system of four vessels, each holding 2,000 lb of GAC, is approximately $\$ 57,000$. Carbon replacement costs are currently approximately $\$ 2-3 / \mathrm{lb}$. As reported by Darrell Oentrich, a city representative, 
each Barnes public supply well has the capacity to pump at a rate of $90 \mathrm{gpm}$. The wells are generally rotated every other day. Based on the current pumping rate and use, it is estimated that approximately $60-80 \mathrm{lb}$ of carbon will be spent per quarter of operation. The anticipated replacement cost for the GAC in the four-vessel system proposed is estimated at $\$ 1,000$ per year. (calculated on the basis of using $320 \mathrm{lb} /$ year at \$3/lb). Costs for the building and installation will be determined during development of the treatment system design document.

\subsection{Proposed Working Schedule}

The proposed working schedule for treatment system installation is shown in Table 6.2. Implementation of this schedule will be initiated in accord with Kansas regulation K.A.R. 28-15, as discussed in Section 4.2.

\subsection{Potential Implementation Issues}

The following potential implementation issues have been identified:

- Acquisition of permission from the city of Barnes for access to the public property adjacent to the public water supply wellheads for installation of the treatment system. Permission will be required to install a building that will house the GAC unit. Details will be presented in the formal treatment system design document.

- Approval from the city to access and modify the existing water distribution system, as necessary, to accommodate the GAC equipment. 
TABLE 6.2 Working schedule for treatment system installation. ${ }^{a}$

Milestone

Engineering design and approval phase (concurrent with evaluation)

Conduct community relations and outreach activities, as needed to facilitate IM wellhead treatment design (and implementation)

Conduct access negotiations with city, as needed to facilitate IM treatment design (and implementation)

Contract for engineering design support

Develop draft treatment system engineering design and OMM plans; identify permit requirements

Submit draft design and OMM plans for CCC/USDA and regulatory approval

Amend draft design and plans as necessary to meet CCC/USDA and regulatory requirements

Receive approvals for the engineering design and OMM plans

Identify qualified candidate(s) for supply and installation of the treatment system, per the approved design

Prepare permit application documents for implementation

IM implementation phase (subject to K.A.R. 28-15, as discussed in Section 4.2, and to CCC/USDA and regulatory approval)

Obtain required permits and access agreements

Contract for treatment system supply and installation

Install treatment system, per approved engineering design

Initiate treatment operations and implement approved OMM plans

a Implementation of this schedule will be initiated in accord with Kansas regulation K.A.R. 28-15, as discussed in Section 4.2. 


\section{Status of Treatment System Design Appendices}

\subsection{Treatment System Appendix A: Data Acquisition Plan}

The data acuisition plan will be included in the treatment system design document if it is determined to be a necessary component of the treatment system.

\subsection{Treatment System Appendix B: Quality Assurance Project Plan}

The Argonne Master Work Plan (Argonne 2002) has been submitted to the KDHE. This document includes the quality assurance project plan.

\subsection{Treatment System Appendix C: Treatability Study Testing Plan}

At this time, no treatability study activities are proposed.

\subsection{Treatment System Appendix D: Health and Safety Plan}

The health and safety plan for implementation of the approved contingency IM design will be provided by the implementing contractor and will be submitted in full to the KDHE. A synopsis will be included in the treatment system design document.

\subsection{Treatment System Appendix E: Operations and Maintenance Plan}

The operations and maintenance plan will be provided with the treatment system design. 


\section{References}

AEI, 2000, Quarterly Sampling Summary Report for Westside Service, Barnes, Kansas — Fourth Event, prepared for Bureau of Environmental Remediation, Kansas Department of Health and Environment, Topeka, Kansas, by Associated Environmental, Inc., Manhattan, Kansas, November 14.

Argonne, 2002, Final Master Work Plan: Environmental Investigations at Former CCC/USDA Facilities in Kansas, 2002 Revision, ANL/ER/TR-02/004, prepared for the Commodity Credit Corporation, U.S. Department of Agriculture, Washington, D.C., by Argonne National Laboratory, Argonne, Illinois, December.

Argonne, 2006, Documentation for Refined Property Boundaries for the Former CCC/USDA Grain Storage Facility at Barnes, Kansas, ANL/EVS/AGEM/CHRON-941, prepared for the Commodity Credit Corporation, U.S. Department of Agriculture, Washington, D.C., by Argonne National Laboratory, Argonne, Illinois, March 7.

Argonne, 2008a, Final Report: Results of the 2007-2007 Investigation of Potential Contamination at the Former CCC/USDA facility in Barnes, Kansas, ANL/EVS/AGEM/TR-0712, prepared for the Commodity Credit Corporation, U.S. Department of Agriculture, Washington, D.C., by Argonne National Laboratory, Argonne, Illinois, August.

Argonne, 2008b, November 2007 Monitoring Results for Barnes, Kansas, ANL/EVS/AGEM/TR-08-04, prepared for the Commodity Credit Corporation, U.S. Department of Agriculture, Washington, D.C., by Argonne National Laboratory, Argonne, Illinois, February.

Argonne, 2008c, March 2008 Monitoring Results for Barnes, Kansas, ANL/EVS/AGEM/TR-0811, prepared for the Commodity Credit Corporation, U.S. Department of Agriculture, Washington, D.C., by Argonne National Laboratory, Argonne, Illinois, August.

Argonne, 2008d, July 2008 Monitoring Results for Barnes, Kansas, ANL/EVS/AGEM/TR-0817, prepared for the Commodity Credit Corporation, U.S. Department of Agriculture, Washington, D.C., by Argonne National Laboratory, Argonne, Illinois, September. 
BE\&K, 1999, Public Water Supply Site Comprehensive Investigation Barnes, Kansas, prepared for the Kansas Department of Health and Environment, Topeka, Kansas, by BE\&K/Terranext, March.

BE\&K, 2000, March 2000 Quarterly Monitoring Report: Barnes Public Water Supply Site Barnes, Kansas, prepared for the Kansas Department of Health and Environment, Topeka, Kansas, by BE\&K/Terranext, May.

Johnson, D.L., 1989, letter from Johnson (Superintendent, Unified School District 223, BarnesHanover-Linn, Barnes, Kansas) to J. Goetz (Bureau of Air and Waste Management, Kansas Department of Health and Environment, Topeka, Kansas), received October 16, with attachment (USD 223 [1989]).

KDHE, 1990, KDHE Incident Notification Report for Fertilizer Spill at Barnes Co-op on October 23, 1990, Kansas Department of Health and Environment, Topeka, Kansas, October 24.

KDHE, 1997a, memorandum from B. Cimino to S. Long, Bureau of Environmental Remediation, Kansas Department of Health and Environment, Topeka, Kansas, regarding October 1997 sampling of public water supply wells in Barnes, Kansas, September 12.

KDHE, 1997b, memorandum from B. Cimino (through S. Stover and F. Arnwine) to R. Bean, Bureau of Environmental Remediation, Kansas Department of Health and Environment, Topeka, Kansas, regarding reclassification of the Barnes PWS2-PWS3 site, October 14. PRC, 1996, Barnes Public Water Supply Site, Barnes, Washington County, Final Phase I Comprehensive Investigation Report, prepared for the Kansas Department of Health and Environment, Topeka, Kansas, by PRC Environmental Management, Inc., August.

KDHE, 1998a, data transmittal for Barnes facility, Kansas Department of Health and Environment Bureau of Environmental Remediation: USDA/CCC Private Well Sampling Program.

KDHE, 1998b, data transmittal for Barnes south facility, Kansas Department of Health and Environment Bureau of Environmental Remediation: USDA/CCC Private Well Sampling Program. 
KDHE, 2001a, transmittal of data files for Barnes, Kansas, from D. Porter (Bureau of Environmental Remediation, Kansas Department of Health and Environment, Topeka, Kansas) to C. Roe (Commodity Credit Corporation, U.S. Department of Agriculture, Washington, D.C.), April 5.

KDHE, 2001b, Waste Chemical Disposal Guidance for Schools, Bureau of Waste Management, Kansas Department of Health and Environment, Topeka, Kansas, October (www.kdheks.gov/waste/download/school_chemical_guidance_book_1001.pdf).

KDHE, 2002, transmittal of analytical results for sampling at Barnes, Kansas, on March 8, 2002, from D. Porter (Bureau of Environmental Remediation, Kansas Department of Health and Environment, Topeka, Kansas) to C. Roe (Commodity Credit Corporation, U.S. Department of Agriculture, Washington, D.C.), April 8.

KDHE, 2003, “Kansas School Lab Sweep Program,” page 3 in Solid Waste Update, Volume 7, Number 2, November (www.kdheks.gov/waste/swupdate/SW_Update_1103.pdf).

KDHE, 2006a, Policy and Scope of Work: Interim Measures, BER Policy \#BER-RS-029, Bureau of Environmental Remediation/Remedial Section, Kansas Department of Health and Environment, Topeka, Kansas, revised October 2006 (http://www.kdheks.gov/ber/policies/ BER_RS_029.pdf).

KDHE, 2006b, electronic mail message and analytical data file (2000 to transmittal date) for Barnes, Kansas, from C. Carey (Bureau of Environmental Remediation, Kansas Department of Health and Environment, Topeka, Kansas) to L.M. LaFreniere (Environmental Science Division, Argonne National Laboratory, Argonne, Illinois), March 28.

KDHE, 2007, data transmittal from M. Carpenter (Municipal Programs Section, Bureau of Water, Kansas Department of Health and Environment, Topeka, Kansas) to L. Larsen (Larsen and Associates, Inc., Lawrence, Kansas), November.

KDHE, 2008a, letter from E. Finzer (Bureau of Environmental Remediation, Kansas Department of Health and Environment, Topeka, Kansas) to C. Roe (Commodity Credit Corporation, U.S. Department of Agriculture, Washington, D.C., December 30. 
KDHE, 2008b, telephone conversation between B. Tavener (Unit Chief, Wellhead Protection Unit, Storage Tank Section, Kansas Department of Health and Environment, Topeka Kansas) and Lisa Larsen (Larsen and Associates, Inc., Lawrence, Kansas), November 12.

NAIP, 2004, aerial photograph of Barnes, Kansas, National Aerial Imagery Program, U.S. Department of Agriculture (http://www.apfo.usda.gov/NAIP.html).

NAPP, 2002, aerial image NAPP 12897 222, National Aerial Photography Program, U.S. Geological Survey (available from Kansas Geospatial Community Commons [http://www.kansasgis.org/]), April 9.

PRC, 1996, Barnes Public Water Supply Site, Barnes, Washington County, Final Phase I Comprehensive Investigation Report, prepared for the Kansas Department of Health and Environment, Topeka, Kansas, by PRC Environmental Management, Inc., August.

USD 223, 1989, Inventory of Chemical Waste Accumulated, transmitted from D.L. Johnson (district superintendent) to J. Goetz (Hazardous Waste Section, Kansas Department of Health and Environment, Topeka, Kansas).

USDA, 1962, aerial photograph YV-1CC-55, U.S. Department of Agriculture, Farm Service Agency, Aerial Photography Field Office, Salt Lake City, Utah, July 25.

Wurtz, L., 2008, telephone conversation between Wurtz (Transportation/Food Service Supervisor, Unified School District 223, Barnes, Kansas) and L. Larsen (Larsen and Associates, Inc., Lawrence, Kansas), November. 


\section{Appendix A:}

Technical Analysis of the Barnes School as a Potentially Responsible Party 


\section{Appendix A:}

\section{Technical Analysis of the Barnes School as a Potentially Responsible Party}

Historical detections of carbon tetrachloride in the public water supply wells at Barnes are consistent with two independent sources of contamination, resulting from

- Past releases near the school's agriculture building, located within $200 \mathrm{ft}$ of wells PWS2 and PWS3.

- Past releases at the former CCC/USDA grain storage facility, located approximately $800 \mathrm{ft}$ east-southeast of the two public wells.

Figure 1 shows the potential source areas investigated by the KDHE during 1996-1999. Elevated levels of carbon tetrachloride in soil gas are evident at the school (near the former agriculture building/automotive shop and the art building) and at the former CCC/USDA grain storage facility. The basis for identifying the school as a PRP rests on

- The findings of the KDHE's 1996 soil gas investigation, which included identification of an uncontaminated area separating the two source areas at the school and the former CCC/USDA facility, and

- Documented school activities related to releases of chemicals near the two public wells.

The pattern of carbon tetrachloride contamination apparent in available data for the two public water supply wells from 1986 to the present (Table A.1) is consistent with a scenario in which a release near the public wells (the school source) resulted in the highest known concentrations in the earliest years of monitoring ( $>2 \mu \mathrm{g} / \mathrm{L}$ in 1986-1987). No data are available to indicate whether higher levels were present in prior years. Subsequent lower sporadic detections (not detected to approximately $1 \mu \mathrm{g} / \mathrm{L}$ in 1988-2000) suggest the depletion of the nearby school source. The trace levels of contamination identified in the recent sampling from 2007 to the present might represent the leading edge of the contaminant plume from the more 
distant source (the CCC/USDA facility) as it is drawn toward the public wells because of their pumping influence.

The sections below summarize the KDHE documentation regarding two independent sources of carbon tetrachloride.

\section{A.1 KDHE Conclusion Regarding Two Independent Sources of Carbon Tetrachloride}

In the KDHE’s 1996 Phase I comprehensive investigation (PRC 1996), soil gas samples were collected from both potential source areas (the school and the former CCC/USDA facility) and at the city park between the two. Figure 5 in the 1996 report (PRC 1996) showed the results of that soil gas investigation. (See also Figure A.1 of this appendix.) The KDHE's conclusions (page 36 in PRC [1996]) stated the following (quoting the original, with italics added for emphasis):

Based on the soil gas results, the former high school and the former [CCC/USDA] grain bin facility appear to be two independent sources of carbon tetrachloride contamination. Both potential sources had several positive soil gas results, indicating carbon tetrachloride contamination at both areas, while the results showing no detections in the samples taken from the city park indicate that the two contaminated areas are not connected.

Soil gas sampling results at the former [CCC/USDA] grain bin facility indicate that carbon tetrachloride contamination has not migrated in soil gas from the potential source. The results from two sample locations on the north side of the former grain bin facility and one sample location to the east showed no detections. This indicates that contamination has not migrated to the north or east from the site. The non-detection results in City Park show that contamination has not migrated west from the former [CCC/USDA] grain bin facility or east from the former high school.

[Groundwater] sampling results indicate that carbon tetrachloride contamination has not migrated extensively in groundwater from either potential source. In the past, carbon tetrachloride has been detected in the [public water supply] wells intermittently. The intermittent hits indicate that carbon tetrachloride contamination has not migrated in groundwater to the north or east from the former high school, but that under the right conditions, pumping of the public water supply wells pulls carbon tetrachloride contamination from the source area [i.e., the school] into the wells. 
The KDHE contractor, PRC, recommended continued monitoring of the public wells, stating (page 37 in PRC [1996]), "through continued monitoring, any increase in the level of contaminants will be detected in a timely manner. The level of contaminants is not anticipated to be cause for concern in the future because the two potential sources are not active and levels of carbon tetrachloride in the [public water supply] wells have not exceeded maximum contaminant levels in the past. Also, mixing of water from the two [public water supply] wells in the water tower would likely cause further dilution of the low carbon tetrachloride concentrations.”

\section{A.2 KDHE Documentation of the School as a PRP}

In the 1996 Phase I comprehensive investigation (PRC 1996), the KDHE investigated two source areas for the carbon tetrachloride contamination: (1) the former high school and (2) the former CCC/USDA grain storage facility. The KDHE identified the school as a potential source area because of documentation regarding the school's storage and use of numerous hazardous chemicals, including carbon tetrachloride and chloroform, as well as documentation that chemicals were improperly released to the ground outside the school.

Although no documentation exists regarding the exact locations of disposal of chemicals, the KDHE did document the general location of disposal and its proximity to the public wells. The following documentation regarding the use and disposal of chemicals by the school and the location of the school source area(s) was identified in the Phase I comprehensive investigation report (PRC 1996):

- The former high school auditorium is approximately $120 \mathrm{ft}$ east of the two public wells. The remainder of the building (the former high school building) was torn down in about 1978. A separate T-shaped wooden building, located about $50 \mathrm{ft}$ southwest of the auditorium, formerly served as the agricultural vocational building. According to Mr. Flear, a lifelong resident of Barnes, chemicals were mixed in the building and dumped outside when they were no longer needed (pages 4-6 and Appendix A in PRC [1996]).

- Mr. Flear told Scott Alberg (the KDHE project manager for the PRC investigation) that the white building southwest of the high school was the 
agriculture building, the location where chemicals for the school were stored (Appendix A in PRC [1996]).

- KDHE files show that a representative from Unified School District 223 contacted the KDHE in 1989 regarding the proper disposal of numerous chemicals stored in the high school (Johnson 1989; USD 223 1989). According to the comprehensive investigation report (page 6 in PRC [1996]), the wastes still stored at the school at that time included 2 pints of carbon tetrachloride and 2.5 pints of chloroform. (Note that the school's inventory of chemicals included by the KDHE as part of the project record listed 2.25 pints of chloroform, not the 2.5 pints stated by PRC [1996].)

\section{A.3 Chemical Waste in Schools Is a Widespread Concern}

Chemical use in schools is most typically associated with maintenance areas, vocational education shops, science laboratories, and art classrooms. State and federal regulatory agencies have recognized improper storage, use, and disposal of chemicals in schools as a safety and environmental hazard. During the Kansas School Lab Sweep Program implemented by the KDHE to remove unwanted or obsolete chemicals from schools (KDHE 2003), thousands of containers of hazardous waste were removed for proper disposal. The KDHE Bureau of Waste Management issued guidance (KDHE 2001b) to help school administrators, teachers, and custodians dispose of unwanted chemicals.

\section{A.4 Potential Source Areas Associated with Barnes School Curriculum Activities}

Although the specific release of carbon tetrachloride by the Barnes school is not documented, the documentation cited above confirms the following:

- Carbon tetrachloride was used as part of the Barnes school curriculum activities.

- Chemicals were stored in the former agriculture building. 
- Waste chemicals were improperly released to the ground adjacent to the former agriculture building.

The following facts are consistent with release of carbon tetrachloride as part of school activities in the identified area near the public wells:

- The known school activities in the disposal area included vocational training in agriculture and automotive maintenance at the former agriculture building (Wurtz 2008) and art classes in the building located to the east.

- The common occurrence of carbon tetrachloride as a component in fumigants, solvents, and paints during the years when the school was in operation.

- The elevated levels of carbon tetrachloride in soil gas found in the KDHE's early investigation of the school source areas (PRC 1996). 
TABLE A.1 Carbon tetrachloride concentrations in the public water supply wells at Barnes in 1986-2008.

\begin{tabular}{|c|c|c|c|}
\hline \multirow{2}{*}{$\begin{array}{c}\text { Sample } \\
\text { Date }\end{array}$} & \multicolumn{2}{|c|}{$\begin{array}{c}\text { Concentration }(\mu \mathrm{g} / \mathrm{L}) \text { in } \\
\text { Indicated Well }\end{array}$} & \multirow{2}{*}{$\begin{array}{l}\text { Reporting } \\
\text { Limit }(\mu \mathrm{g} / \mathrm{L})\end{array}$} \\
\hline & PWS2 & PWS3 & \\
\hline $4 / 8 / 86$ & 2.1 & 0.5 & Unknown \\
\hline $4 / 22 / 86$ & 1.3 & 0.2 & Unknown \\
\hline 7/7/87 & 2.5 & 2.1 & Unknown \\
\hline $1 / 7 / 88$ & $N D^{a}$ & ND & Unknown \\
\hline $9 / 2 / 88$ & ND & ND & Unknown \\
\hline 9/22/88 & ND & ND & Unknown \\
\hline $1 / 30 / 89$ & ND & ND & Unknown \\
\hline 7/13/89 & ND & ND & Unknown \\
\hline 1990 & No data & No data & - \\
\hline $8 / 12 / 91$ & ND & 0.5 & 0.5 \\
\hline 1992 & No data & No data & - \\
\hline 1993 & No data & No data & - \\
\hline 1994 & No data & No data & - \\
\hline 4/11/95 & 0.5 & ND & 0.5 \\
\hline $7 / 25 / 95$ & 1.1 & ND & Unknown \\
\hline 5/1/96 & ND & ND & 1.2 \\
\hline 8/13/96 & 1.3 & 0.5 & 0.5 \\
\hline $8 / 27 / 97$ & 0.9 & ND & 0.5 \\
\hline 1998 & No data & No data & - \\
\hline 1/29/99 & ND & ND & 1 \\
\hline $7 / 12 / 99$ & 1.2 & 1.7 & 0.5 \\
\hline $3 / 22 / 00$ & ND & ND & 1 \\
\hline $7 / 11 / 00$ & 0.5 & ND & 0.5 \\
\hline 7/9/01 & ND & ND & 0.5 \\
\hline $7 / 15 / 02$ & ND & ND & 0.5 \\
\hline 2003 & No data & No data & - \\
\hline 2004 & No data & No data & - \\
\hline $7 / 11 / 05$ & ND & ND & 0.5 \\
\hline 2006 & No data & No data & - \\
\hline $3 / 9 / 07$ & ND & $0.2 \mathrm{~J}^{\mathrm{b}}$ & 0.1 \\
\hline 4/5/07 & ND & ND & 0.1 \\
\hline $11 / 20 / 07$ & ND & ND & 0.1 \\
\hline 3/6/08 & ND & ND & 0.1 \\
\hline $7 / 11 / 08$ & $0.8 \mathrm{~J}$ & $0.2 \mathrm{~J}$ & 0.1 \\
\hline
\end{tabular}

a Not detected at the indicated reporting limit.

b Qualifier J indicates an estimated concentration below the purge-and-trap method quantitation limit of $1.0 \mu \mathrm{g} / \mathrm{L}$. 


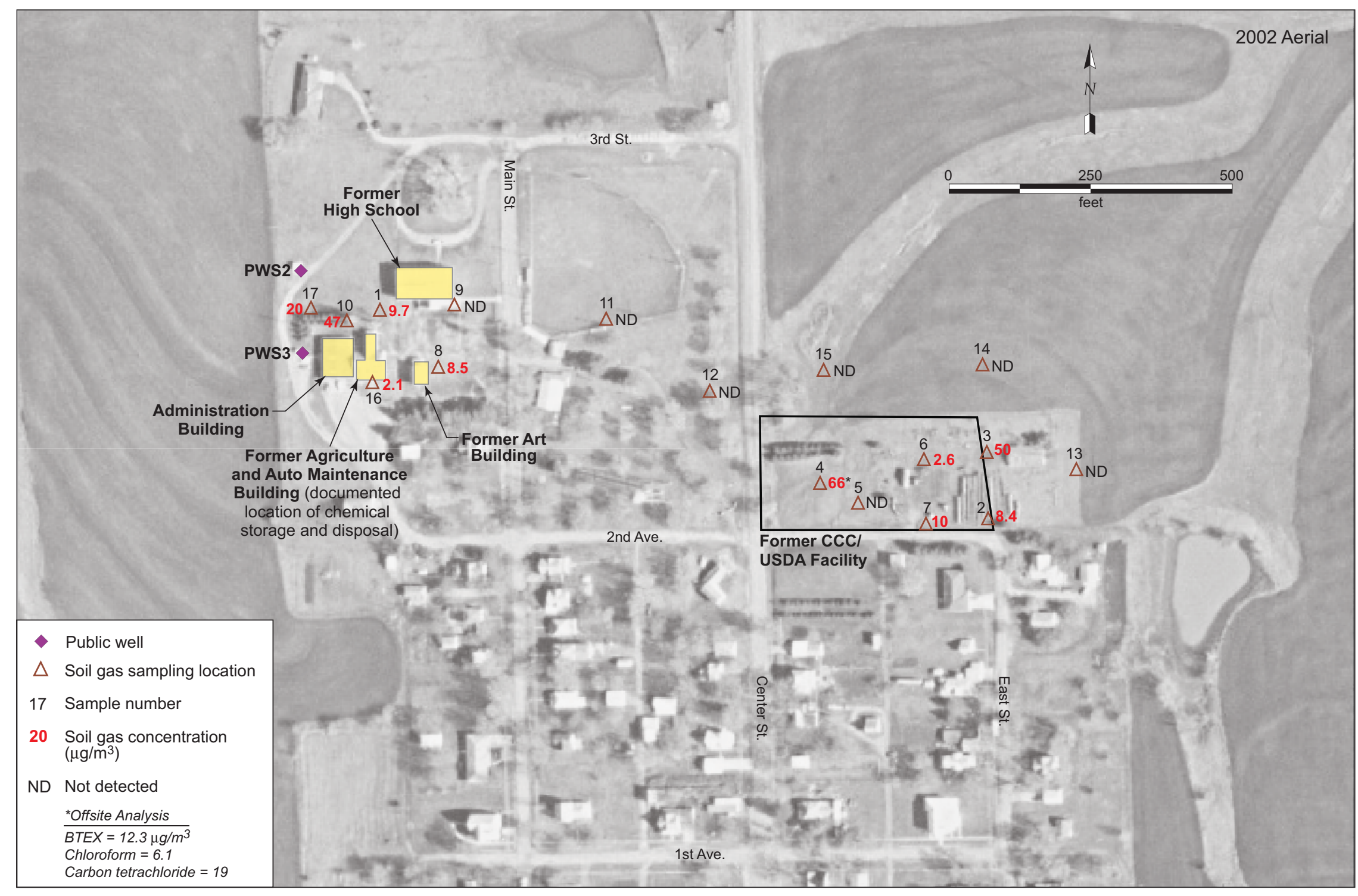

FIGURE A.1 Carbon tetrachloride concentrations in soil gas samples collected by the KDHE in 1996 to investigate two source areas at Barnes. Source of data: PRC (1996). 

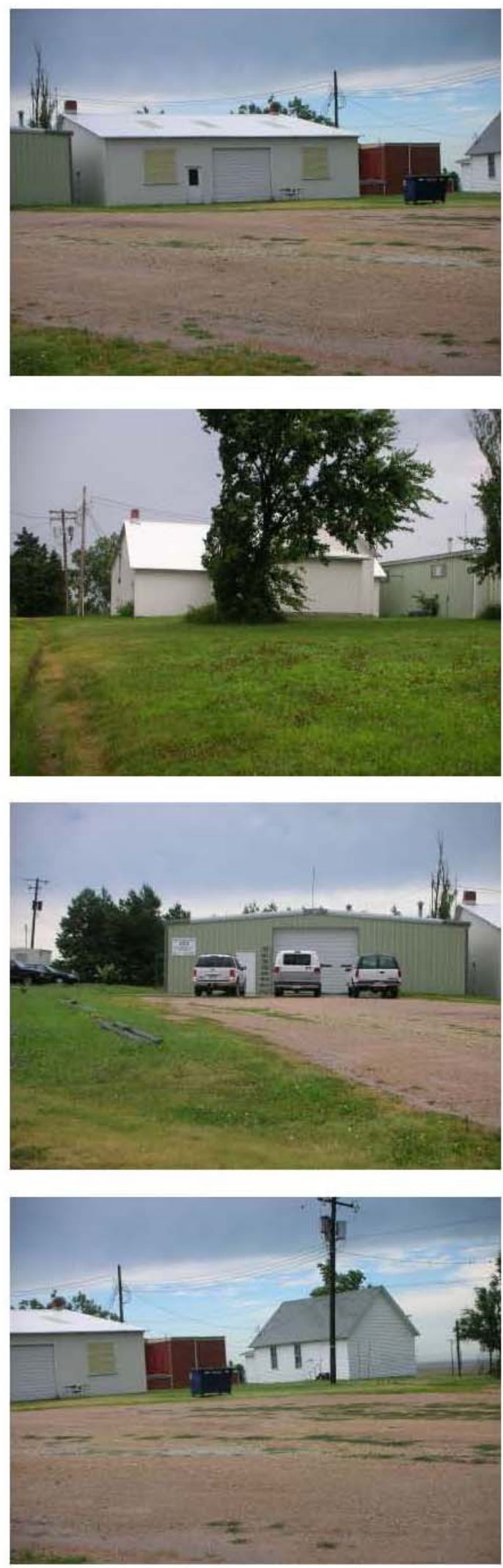

Photo facing northeast. The white building (to the right) was formerly used for art classes.

The red building in the background is the former high school.

The white building to the left is the former Ag building.

FIGURE A.2 The school buildings at Barnes, July 2008. 


\section{Appendix B:}

Historical Analytical Results (1986-2005) and Well Completion Information for the Barnes Public Water Supply 
TABLE B.1 Historical analytical data for wells at Barnes in 1986-2005.

\begin{tabular}{|c|c|c|c|c|c|c|c|c|c|c|c|}
\hline \multirow[b]{3}{*}{ Date } & \multirow[b]{3}{*}{ Location } & \multirow[b]{3}{*}{ Medium $^{\mathrm{a}}$} & \multirow[b]{3}{*}{$\begin{array}{c}\text { Sample } \\
\text { Depth (ft BGL) }\end{array}$} & \multirow{3}{*}{$\begin{array}{l}\text { Depth to } \\
\text { Water } \\
\text { (ft TOC) }\end{array}$} & \multicolumn{6}{|c|}{ Concentration (ppb) } & \multirow[b]{3}{*}{$\begin{array}{c}\text { Nitrates } \\
(\mathrm{ppm})\end{array}$} \\
\hline & & & & & \multicolumn{2}{|c|}{ Field Laboratory Analysis } & \multicolumn{4}{|c|}{ Off-Site Laboratory Analysis } & \\
\hline & & & & & $\begin{array}{c}\text { Carbon } \\
\text { Tetrachloride }\end{array}$ & Chloroform & $\begin{array}{c}\text { Carbon } \\
\text { Tetrachloride }\end{array}$ & Chloroform & $\begin{array}{l}\text { Methylene } \\
\text { Chloride }\end{array}$ & $\begin{array}{l}\text { Total } \\
\text { BTEX }^{b}\end{array}$ & \\
\hline \multicolumn{12}{|c|}{ Historical public well sampling reported by PRC (1996) and KDHE (1997a) and CCC/KDHE private well sampling program } \\
\hline $4 / 8 / 86$ & PWS2 & GW & $155(T D)^{c}$ & $\mathrm{NM}^{\mathrm{d}}$ & $N A^{e}$ & NA & 2.1 & $N D^{f}$ & $N^{g}$ & NR & - \\
\hline $4 / 22 / 86$ & PWS2 & GW & 155 (TD) & NM & NA & NA & 1.3 & ND & NR & NR & - \\
\hline 7/7/87 & PWS2 & GW & 155 (TD) & NM & NA & NA & 2.5 & ND & NR & NR & 10.6 \\
\hline 1/7/88 & PWS2 & GW & 155 (TD) & NM & NA & NA & ND & NR & NR & NR & 10 \\
\hline 9/2/88 & PWS2 & GW & $155(T D)$ & NM & NA & NA & ND & ND & NR & NR & - \\
\hline 9/22/88 & PWS2 & GW & 155 (TD) & NM & NA & NA & ND & NR & NR & NR & 9.4 \\
\hline $1 / 30 / 89$ & PWS2 & GW & $155(T D)$ & NM & NA & NA & ND & 8.7 & NR & $N R^{h}$ & 9.06 \\
\hline 7/13/89 & PWS2 & GW & 155 (TD) & NM & NA & NA & ND & NR & NR & NR & 8.15 \\
\hline 8/12/91 & PWS2 & GW & 155 (TD) & NM & NA & NA & ND & ND & NR & $\mathrm{NR}^{\mathrm{h}}$ & - \\
\hline 4/11/95 & PWS2 & GW & 155 (TD) & NM & NA & NA & 0.5 & ND & NR & NR & - \\
\hline 7/25/95 & PWS2 & GW & 155 (TD) & NM & NA & NA & 1.1 & ND & NR & NR & - \\
\hline 4/8/86 & PWS3 & GW & 160 (TD) & NM & NA & NA & 0.5 & ND & NR & NR & - \\
\hline $4 / 22 / 86$ & PWS3 & GW & 160 (TD) & NM & NA & NA & 0.2 & ND & NR & NR & - \\
\hline 7/7/87 & PWS3 & GW & 160 (TD) & NM & NA & NA & 2.1 & ND & NR & NR & 10.6 \\
\hline 1/7/88 & PWS3 & GW & 160 (TD) & NM & NA & NA & ND & NR & NR & NR & 8.15 \\
\hline 9/2/88 & PWS3 & GW & 160 (TD) & NM & NA & NA & ND & ND & NR & NR & - \\
\hline 9/22/88 & PWS3 & GW & 160 (TD) & NM & NA & NA & ND & NR & NR & NR & 9.4 \\
\hline 1/30/89 & PWS3 & GW & 160 (TD) & NM & NA & NA & ND & 0.8 & NR & NR & 8.11 \\
\hline 7/13/89 & PWS3 & GW & 160 (TD) & NM & NA & NA & ND & 1.5 & NR & NR & - \\
\hline 8/12/91 & PWS3 & GW & 160 (TD) & NM & NA & NA & ND & ND & NR & NR & - \\
\hline 4/11/95 & PWS3 & GW & 160 (TD) & NM & NA & NA & ND & ND & NR & NR & - \\
\hline $7 / 25 / 95$ & PWS3 & GW & 160 (TD) & NM & NA & NA & ND & ND & NR & NR & - \\
\hline \multicolumn{12}{|c|}{ Sampling for the Phase I comprehensive investigation (PRC 1996) } \\
\hline $4 / 30 / 96$ & Probe 1 & SG & $4-6$ & - & $<1$ & $<2$ & NA & NA & NA & NA & NA \\
\hline $4 / 30 / 96$ & Probe 1 & SG & $10-12$ & - & $<1$ & $<2$ & NA & NA & NA & NA & NA \\
\hline $4 / 30 / 96$ & Probe 1 & SG & $16-18$ & - & 9.7 & $<2$ & NA & NA & NA & NA & NA \\
\hline
\end{tabular}




\begin{tabular}{|c|c|c|c|c|c|c|c|c|c|c|c|}
\hline \multirow[b]{3}{*}{ Date } & \multirow[b]{3}{*}{ Location } & \multirow[b]{3}{*}{ Medium $^{\mathrm{a}}$} & \multirow[b]{3}{*}{$\begin{array}{c}\text { Sample } \\
\text { Depth (ft BGL) }\end{array}$} & \multirow{3}{*}{$\begin{array}{l}\text { Depth to } \\
\text { Water } \\
\text { (ft TOC) }\end{array}$} & \multicolumn{6}{|c|}{ Concentration (ppb) } & \multirow[b]{3}{*}{$\begin{array}{l}\text { Nitrates } \\
(\mathrm{ppm})\end{array}$} \\
\hline & & & & & \multicolumn{2}{|c|}{ Field Laboratory Analysis } & \multicolumn{4}{|c|}{ Off-Site Laboratory Analysis } & \\
\hline & & & & & $\begin{array}{c}\text { Carbon } \\
\text { Tetrachloride }\end{array}$ & Chloroform & $\begin{array}{c}\text { Carbon } \\
\text { Tetrachloride }\end{array}$ & Chloroform & $\begin{array}{l}\text { Methylene } \\
\text { Chloride }\end{array}$ & $\begin{array}{l}\text { Total } \\
\text { BTEX }\end{array}$ & \\
\hline \multicolumn{12}{|c|}{ Sampling for the Phase I comprehensive investigation (PRC 1996) (cont.) } \\
\hline $4 / 30 / 96$ & Probe 2 & SG & $4-6$ & - & $<1$ & $<2$ & NA & NA & NA & NA & NA \\
\hline $4 / 30 / 96$ & Probe 2 & SG & $7-9$ & - & 8.4 & $<2$ & NA & NA & NA & NA & NA \\
\hline $4 / 30 / 96$ & Probe 2 & SG & $12-14$ & - & $<1$ & $<2$ & NA & NA & NA & NA & NA \\
\hline $4 / 30 / 96$ & Probe 3 & SG & $6-9$ & - & 50 & $<2$ & NA & NA & NA & NA & NA \\
\hline 4/30/96 & Probe 4 & SG & $4-6$ & - & 66 & 15 & 19 & 6.1 & $<3.5$ & 12.3 & NA \\
\hline 4/30/96 & Probe 5 & SG & $4-6$ & - & $<1$ & $<2$ & NA & NA & NA & NA & NA \\
\hline 4/30/96 & Probe 6 & SG & $4-6$ & - & 2.6 & $<2$ & NA & NA & NA & NA & NA \\
\hline 4/30/96 & Probe 7 & SG & $6-8$ & - & 10 & $<2$ & NA & NA & NA & NA & NA \\
\hline $4 / 30 / 96$ & Probe 8 & SG & $22-24$ & - & 8.5 & 3.5 & NA & NA & NA & NA & NA \\
\hline $4 / 30 / 96$ & Probe 9 & SG & $16-18$ & - & $<1$ & $<2$ & NA & NA & NA & NA & NA \\
\hline $4 / 30 / 96$ & Probe 10 & SG & $16-18$ & - & 47 & 2.6 & 28 & $<2.5$ & $<3.5$ & 2.9 & NA \\
\hline $5 / 1 / 96$ & Probe 11 & SG & $11-13$ & - & $<1$ & $<2$ & NA & NA & NA & NA & NA \\
\hline $4 / 30 / 96$ & Probe 12 & SG & $7-9$ & - & $<1$ & $<2$ & NA & NA & NA & NA & NA \\
\hline 4/30/96 & Probe 13 & SG & $4-6$ & - & $<1$ & $<2$ & NA & NA & NA & NA & NA \\
\hline $5 / 1 / 96$ & Probe 14 & SG & $7-9$ & - & $<1$ & $<2$ & NA & NA & NA & NA & NA \\
\hline $5 / 1 / 96$ & Probe 15 & SG & $4-6$ & - & $<1$ & $<2$ & NA & NA & NA & NA & NA \\
\hline $5 / 1 / 96$ & Probe 16 & SG & $16-18$ & - & 2.1 & $<2$ & NA & NA & NA & NA & NA \\
\hline \multirow[t]{2}{*}{$5 / 1 / 96$} & Probe 17 & SG & $16-18$ & - & 20 & $<2$ & NA & NA & NA & NA & NA \\
\hline & & & & & & & NA & NA & NA & NA & NA \\
\hline $5 / 1 / 96$ & Probe 4 & $\mathrm{~S}$ & $4-6$ & - & $<1$ & $<0.2$ & $<30$ & $<13$ & $<64$ & NA & NA \\
\hline $5 / 1 / 96$ & Probe 3 & $\mathrm{~S}$ & $4-6$ & - & $<1$ & $<0.2$ & $<30$ & $<13$ & $<64$ & NA & NA \\
\hline $5 / 1 / 96$ & Probe 3 Dup & $\mathrm{S}$ & $4-6$ & - & $<1$ & $<0.2$ & $<30$ & $<13$ & $<64$ & NA & NA \\
\hline $5 / 1 / 96$ & Probe 10 & $\mathrm{~S}$ & $17-19$ & - & $<1$ & $<0.2$ & $<30$ & $<13$ & $<64$ & NA & NA \\
\hline $4 / 29 / 96$ & Cooney & GW & Unknown & NM & NA & NA & $<1.2$ & $<0.5$ & $<2.5$ & NA & NA \\
\hline $4 / 29 / 96$ & Oentrich & GW & 150 & NM & NA & NA & $<1.2$ & $<0.5$ & $<2.5$ & NA & NA \\
\hline 4/29/96 & Perkins & GW & 60 & NM & NA & NA & $<1.2$ & $<0.5$ & $<2.5$ & NA & NA \\
\hline $5 / 1 / 96$ & Rothlisberger & GW & 100 & NM & NA & NA & $<1.2$ & $<0.5$ & $<2.5$ & NA & NA \\
\hline $5 / 1 / 96$ & PWS3 & GW & 160 (TD) & NM & NA & NA & $<1.2$ & $<0.5$ & $<2.5$ & NA & NA \\
\hline $5 / 1 / 96$ & PWS2 & GW & 155 (TD) & NM & NA & NA & $<1.2$ & $<0.5$ & $<2.5$ & NA & NA \\
\hline
\end{tabular}




\begin{tabular}{|c|c|c|c|c|c|c|c|c|c|c|c|}
\hline \multirow[b]{3}{*}{ Date } & \multirow[b]{3}{*}{ Location } & \multirow[b]{3}{*}{ Medium $^{\mathrm{a}}$} & \multirow[b]{3}{*}{$\begin{array}{c}\text { Sample } \\
\text { Depth (ft BGL) }\end{array}$} & \multirow{3}{*}{$\begin{array}{l}\text { Depth to } \\
\text { Water } \\
\text { (ft TOC) }\end{array}$} & \multicolumn{6}{|c|}{ Concentration (ppb) } & \multirow[b]{3}{*}{$\begin{array}{l}\text { Nitrates } \\
(\mathrm{ppm})\end{array}$} \\
\hline & & & & & \multirow{2}{*}{\multicolumn{2}{|c|}{$\begin{array}{l}\text { Field Laboratory Analysis } \\
\text { Carbon } \\
\text { Tetrachloride Chloroform }\end{array}$}} & \multicolumn{4}{|c|}{ Off-Site Laboratory Analysis } & \\
\hline & & & & & & & $\begin{array}{c}\text { Carbon } \\
\text { Tetrachloride }\end{array}$ & Chloroform & $\begin{array}{l}\text { Methylene } \\
\text { Chloride }\end{array}$ & $\begin{array}{l}\text { Total } \\
\text { BTEX }^{b}\end{array}$ & \\
\hline
\end{tabular}

Public water supply sampling reported by the KDHE (1997b)

8/13/96 PWS2 $\quad$ GW 155 (TD) NM

$\begin{array}{lllll}8 / 13 / 96 & \text { PWS2 } & \text { GW } & 155 \text { (TD) } & \text { NM } \\ 8 / 13 / 96 & \text { PWS3 } & \text { GW } & 160 \text { (TD) } & \text { NM }\end{array}$

NA

Private well sampling reported by the KDHE $(1998 a, b)$

$\begin{array}{lllll}8 / 28 / 97 & \text { PWS2 } & \text { GW } & 155 \text { (TD) } & \text { NM } \\ 8 / 28 / 97 & \text { PWS3 } & \text { GW } & 160 \text { (TD) } & \text { NM } \\ 6 / 23 / 98 & \text { Finch }^{k} & \text { DW } & \text { Unknown } & \text { NM }\end{array}$

Sampling reported by BE\&K/Terranext (1999)

$\begin{array}{llccc}11 / 30 / 98 & \text { MW-1D } & \text { S } & 21-23 & - \\ 12 / 1 / 98 & \text { MW-2D } & \text { S } & 21-23 & - \\ 12 / 1 / 98 & \text { MW-2D Dup } & \text { S } & 21-23 & - \\ 12 / 1 / 98 & \text { MW-3D } & \text { S } & 15-17 & - \\ 12 / 1 / 98 & \text { MW-4D } & \text { S } & 6-8 & - \\ & & & & \\ 12 / 28 / 98 & \text { MW-1S } & \text { GW } & 13.3-23.3 & \text { Dry } \\ 12 / 28 / 98 & \text { MW-1D } & \text { GW } & 139.85-159.4 & 107.74 \\ 12 / 28 / 98 & \text { MW-2D } & \text { GW } & 133.26-152.93 & 100.61 \\ 12 / 28 / 98 & \text { MW-3D } & \text { GW } & 133.02-152.73 & 97.69 \\ 12 / 28 / 98 & \text { MW-4D } & \text { GW } & 98.38-118.22 & 79.93 \\ & & & & \\ 1 / 29 / 99 & \text { MW-1S } & \text { GW } & 13.3-23.3 & \text { Dry } \\ 1 / 29 / 99 & \text { MW-1D } & \text { GW } & 139.85-159.4 & 109.5 \\ 1 / 29 / 99 & \text { MW-2D } & \text { GW } & 133.26-152.93 & 107.27 \\ 1 / 29 / 99 & \text { MW-3D } & \text { GW } & 133.02-152.73 & 103.71 \\ 1 / 29 / 99 & \text { MW-4D } & \text { GW } & 98.38-118.22 & 84.52 \\ 1 / 29 / 99 & \text { MW-4D Dup } & \text { GW } & 98.38-118.22 & 84.52 \\ 1 / 29 / 99 & \text { Oentrich } & \text { GW } & 150 & \text { NM } \\ 1 / 29 / 99 & \text { PWS2 } & \text { GW } & 155 \text { (TD) } & \text { NM } \\ 1 / 29 / 99 & \text { PWS3 } & \text { GW } & 160 \text { (TD) } & \text { NM } \\ & & & & \end{array}$

NM
NA NA

NA

1.3

0.5

NR

NR

NR

NA

NA

\begin{tabular}{|c|c|c|c|c|c|c|}
\hline NA & NA & 0.9 & $<0.5$ & $<0.5$ & $<0.5$ & NA \\
\hline NA & NA & $<0.5$ & $<0.5$ & $<0.5$ & $<0.5^{\mathrm{h}}$ & NA \\
\hline NA & NA & $<0.5$ & $<0.5$ & $<0.5$ & $<0.5$ & $<20$ \\
\hline
\end{tabular}

NA

NA
$<20$

NA

$<0.5$

$<0.5$

$<0.5$

$<0.5$

$\begin{array}{ll}\text { NA } & \text { NA } \\ \text { NA } & \text { NA } \\ \text { NA } & \text { NA } \\ \text { NA } & \text { NA } \\ \text { NA } & \text { NA } \\ \text { NS } & \text { NS } \\ \text { NA } & \text { NA } \\ \text { NA } & \text { NA } \\ \text { NA } & \text { NA } \\ \text { NA } & \text { NA } \\ & \\ \text { NS } & \text { NS } \\ \text { NA } & \text { NA } \\ \text { NA } & \text { NA } \\ \text { NA } & \text { NA } \\ \text { NA } & \text { NA } \\ \text { NA } & \text { NA } \\ \text { NA } & \text { NA } \\ \text { NA } & \text { NA } \\ \text { NA } & \text { NA }\end{array}$

$\begin{aligned} &< 6.25 \\ &<6.4 \\ &<5.55 \\ &<6.6 \\ &<6.25 \\ & \quad \text { NS } \\ &<1 \\ &<1 \\ &<1 \\ &<1 \\ & \\ & N S \\ &<1 \\ &<1 \\ &<1 \\ &<1 \\ &<1 \\ &<1 \\ &<1 \\ &<1 \\ &<1\end{aligned}$

$<6.25$
$<6.4$
$<5.55$
$<6.6$
$<6.25$

$\quad$ NS
$<1$
$<1$
$<1$
$<1$
$\quad$

$N S$
$<1$
$<1$
$<1$
$<1$
$<1$
$<1$
$<1$
$<1$
$<1$

$<6.25^{\mathrm{i}}$

$<6.4$
$<5.55$

NA

NA

$\begin{array}{lllll}<6.4 & <6.4 & <6.4 & <6.4 & \text { NA } \\ <5.55 & <5.55 & <5.55 & <5.55 & \text { NA }\end{array}$

$<6.6<6.6<6.6<6.6 \quad$ NA

$\begin{array}{lll}<6.25 & <6.25<6.25<6.25 \quad N A\end{array}$

NS NS NS NS NS

$<1$

$<1$

4

$<1$

$<1$

$<1$

NA

NA

NA

NS

$<1$
$<1$

$<1$
$<1$

3.9

$<1$

$<1$
$<1$

$<1$

NS

NA

NA

NA

NA

NA

NA

NA
NA 


\begin{tabular}{|c|c|c|c|c|c|c|c|c|c|c|c|}
\hline \multirow[b]{3}{*}{ Date } & \multirow[b]{3}{*}{ Location } & \multirow[b]{3}{*}{ Medium ${ }^{\mathrm{a}}$} & \multirow[b]{3}{*}{$\begin{array}{c}\text { Sample } \\
\text { Depth (ft BGL) }\end{array}$} & \multirow{3}{*}{$\begin{array}{l}\text { Depth to } \\
\text { Water } \\
\text { (ft TOC) }\end{array}$} & \multicolumn{6}{|c|}{ Concentration (ppb) } & \multirow[b]{3}{*}{$\begin{array}{l}\text { Nitrates } \\
(\mathrm{ppm})\end{array}$} \\
\hline & & & & & \multirow{2}{*}{\multicolumn{2}{|c|}{$\begin{array}{l}\text { Field Laboratory Analysis } \\
\text { Carbon } \\
\text { Tetrachloride Chloroform }\end{array}$}} & \multicolumn{4}{|c|}{ Off-Site Laboratory Analysis } & \\
\hline & & & & & & & $\begin{array}{c}\text { Carbon } \\
\text { Tetrachloride }\end{array}$ & Chloroform & $\begin{array}{l}\text { Methylene } \\
\text { Chloride }\end{array}$ & $\begin{array}{l}\text { Total } \\
\text { BTEX }\end{array}$ & \\
\hline
\end{tabular}

Sampling of wells reported by the KDHE (2001a, 2007)

$\begin{array}{llccc}5 / 4 / 99 & \text { MW-1S } & \text { GW } & 13.3-23.3 & \text { D } \\ 7 / 12 / 99 & \text { PWS2 } & \text { GW } & 155 \text { (TD) } & \text { NM }\end{array}$

7/12/99 PWS3 GW 160 (TD) NM

Dry NS

NA NS

NS

NA

NA

NS
1.2
1.7




$N S$
$<1$
$<1$
$<1$
5.1
4.9
$<1$
$<1$

NS
$<0.5$
0.6





$N S$
$\quad<1$
$<1$
$<1$
$<1$
$<1$
$<1$
1.9

\section{NS}

$<0.5$

NS

$<0.5$
$<0.5$

NA

Sampling reported by BE\&K/Terranext (2000)

$\begin{array}{ll}3 / 22 / 00 & M W-1 S \\ 3 / 22 / 00 & M W-1 D \\ 3 / 22 / 00 & M W-2 D \\ \text { 93/22/00 } & \text { MW-3D } \\ 3 / 22 / 00 & \text { MW-4D } \\ 3 / 22 / 00 & \text { MW-4D Dup } \\ 3 / 22 / 00 & \text { PWS2 } \\ 3 / 22 / 00 & \text { PWS3 }\end{array}$

$\begin{array}{ccc}\text { GW } & 13.3-23.3 & \text { Dry } \\ \text { GW } & 139.85-159.4 & 119.09 \\ \text { GW } & 133.26-152.93 & 116.8 \\ \text { GW } & 133.02-152.73 & 113.69 \\ \text { GW } & 98.38-118.22 & 95.21 \\ \text { GW } & 98.38-118.22 & 95.21 \\ \text { GW } & 155(\text { TD) } & \text { NM } \\ \text { GW } & 160(\text { TD) } & \text { NM }\end{array}$

$\begin{array}{ll}\text { NS } & \text { NS } \\ \text { NA } & \text { NA } \\ \text { NA } & \text { NA } \\ \text { NA } & \text { NA } \\ \text { NA } & \text { NA } \\ \text { NA } & \text { NA } \\ \text { NA } & \text { NA } \\ \text { NA } & \text { NA }\end{array}$

Sampling for Westside Service underground storage tank site reported by AEI (2000)

\begin{tabular}{|c|c|c|c|c|c|c|c|c|c|c|c|}
\hline $10 / 17 / 00$ & MW-1 & GW & $10-45$ & 15.8 & NA & NA & NA & NA & NA & 12800 & NS \\
\hline $10 / 17 / 00$ & MW-2 & GW & 10-45 & 16.19 & NA & NA & NA & NA & NA & 396 & NS \\
\hline $10 / 17 / 00$ & MW-3 & GW & $10-40$ & 19.94 & NS & NS & NS & NS & NS & NS & NS \\
\hline $10 / 17 / 00$ & MW-4 & GW & 10-45 & $14.02^{1}$ & NS & NS & NS & NS & NS & NS & NS \\
\hline $10 / 17 / 00$ & MW-5 & GW & $10-50$ & 44.22 & NA & NA & NA & NA & NA & ND & NS \\
\hline $10 / 17 / 00$ & MW-7 & GW & $24.5-64.5$ & 62.67 & NA & NA & NA & NA & NA & ND & NS \\
\hline $10 / 17 / 00$ & MW-8 & GW & $23.5-63.5$ & 53.4 & NA & NA & NA & NA & NA & ND & NS \\
\hline 10/17/00 & MW-9 & GW & 40.80 & 63.12 & NA & NA & NA & NA & NA & ND & NS \\
\hline \multicolumn{12}{|c|}{ Sampling reported by the KDHE (2007) } \\
\hline 7/11/00 & PWS2 & GW & 155 (TD) & NM & NA & NA & 0.5 & NA & $<0.5$ & $<0.5$ & NA \\
\hline $7 / 11 / 00$ & PWS3 & GW & 160 (TD) & NM & NA & NA & $<0.5$ & NA & 0.5 & $<0.5$ & NA \\
\hline 7/9/01 & PWS2 & GW & 155 (TD) & NM & NA & NA & $<0.5$ & NA & $<0.5$ & $<0.5$ & NA \\
\hline $7 / 9 / 01$ & PWS3 & GW & 160 (TD) & NM & NA & NA & $<0.5$ & NA & $<0.5$ & $<0.5$ & NA \\
\hline
\end{tabular}




\begin{tabular}{|c|c|c|c|c|c|c|c|c|c|c|c|}
\hline \multirow[b]{3}{*}{ Date } & \multirow[b]{3}{*}{ Location } & \multirow[b]{3}{*}{ Medium $^{\mathrm{a}}$} & \multirow[b]{3}{*}{$\begin{array}{c}\text { Sample } \\
\text { Depth (ft BGL) }\end{array}$} & \multirow[b]{3}{*}{$\begin{array}{l}\text { Depth to } \\
\text { Water } \\
\text { (ft TOC) }\end{array}$} & \multicolumn{6}{|c|}{ Concentration (ppb) } & \multirow[b]{3}{*}{$\begin{array}{l}\text { Nitrates } \\
(\mathrm{ppm})\end{array}$} \\
\hline & & & & & \multicolumn{2}{|c|}{ Field Laboratory Analysis } & \multicolumn{4}{|c|}{ Off-Site Laboratory Analysis } & \\
\hline & & & & & $\begin{array}{c}\text { Carbon } \\
\text { Tetrachloride }\end{array}$ & Chloroform & $\begin{array}{c}\text { Carbon } \\
\text { Tetrachloride }\end{array}$ & Chloroform & $\begin{array}{l}\text { Methylene } \\
\text { Chloride }\end{array}$ & $\begin{array}{l}\text { Total } \\
\text { BTEX }^{b}\end{array}$ & \\
\hline \multicolumn{12}{|c|}{ Sampling reported by the KDHE (2002) } \\
\hline 3/8/02 & MW-4D & GW & $98.38-118.22$ & NR & NA & NA & $7.3^{\mathrm{m}}$ & $<1$ & $<0.9$ & $<0.4$ & NA \\
\hline \multicolumn{12}{|c|}{ Sampling of public water supply wells, reported by the KDHE $(2006,2007)$} \\
\hline $7 / 15 / 02$ & PWS2 & GW & 155 (TD) & NR & NA & NA & $<0.5$ & NA & $<0.5$ & $<0.5$ & NR \\
\hline $7 / 15 / 02$ & PWS3 & GW & 160 (TD) & NR & NA & NA & $<0.5$ & NA & $<0.5$ & $<0.5$ & NR \\
\hline 7/11/05 & PWS2 & GW & 155 (TD) & NR & NA & NA & $<0.5$ & NA & $<0.5$ & $<0.5$ & NR \\
\hline $7 / 11 / 05$ & PWS3 & GW & 160 (TD) & NR & NA & NA & $<0.5$ & NA & $<0.5$ & $<0.5$ & NR \\
\hline
\end{tabular}

a Medium types: DW, drinking water; GW, groundwater; S, soil; SG, soil gas.

${ }^{b}$ BTEX: benzene toluene, ethylbenzene, and xylene.

c TD: total depth.

${ }^{\mathrm{d}} \mathrm{NM}$ : not measured.

NA: sample not analyzed for this constituent.

${ }^{f} \mathrm{ND}$ : contaminant not detected.

${ }^{g}$ NR: result not reported in investigation reports on file.

$\mathrm{h}$ NR: result not reported in investigation reports on file.
Trihalomethane compounds other than chloroform reported at trace concentrations.

'Acetone present at low concentration is likely a laboratory contaminant.

NS: no sample (dry well).

${ }^{k}$ Site of Barnes south former CCC/USDA facility.

'Not sampled because of the presence of free product.

$\mathrm{m}$ Sample received by the analytical laboratory at a temperature of $15^{\circ} \mathrm{C}$ (KDHE 2002). 
TABLE B.2 Construction summary for wells at Barnes.

\begin{tabular}{|c|c|c|c|c|c|c|c|c|c|c|c|}
\hline \multirow[b]{2}{*}{$\begin{array}{l}\text { Well } \\
\text { Name }\end{array}$} & \multirow[b]{2}{*}{ Well Location } & \multirow[b]{2}{*}{$\begin{array}{l}\text { WWC-5 } \\
\text { Filed }\end{array}$} & \multirow{2}{*}{$\begin{array}{c}\text { Well } \\
\text { Diameter } \\
\text { (in.) }\end{array}$} & \multirow[b]{2}{*}{$\begin{array}{l}\text { Casing } \\
\text { Type }\end{array}$} & \multicolumn{2}{|c|}{ Elevation (ft AMSL) } & \multirow{2}{*}{$\begin{array}{l}\text { Screen } \\
\text { Length } \\
\text { (ft) }\end{array}$} & \multicolumn{3}{|c|}{ Depth (ft BGL) } & \multirow[b]{2}{*}{$\begin{array}{l}\text { Completion } \\
\text { Date }\end{array}$} \\
\hline & & & & & Ground & Casing & & Screen & Filter & Total & \\
\hline \multicolumn{12}{|c|}{ Public wells (reported by KDHE 1997a) } \\
\hline PWS2 & $1000 \mathrm{ft}$ west of CCC/USDA facility & No & 10 & Steel & $U_{n k}^{b}$ & Unk & Unk & Unk & Unk & 155 & 1928 \\
\hline PWS3 & $1000 \mathrm{ft}$ west of CCC/USDA facility & No & 8 & Steel & Unk & Unk & Unk & Unk & Unk & 160 & 1955 \\
\hline \multicolumn{12}{|c|}{ Private wells (well depths for Oentrich, Perkins, and Rothlisberger wells, as reported by PRC [1996]) } \\
\hline Cooney & $\begin{array}{l}5 / 8 \text { mi west-southwest of CCC/USDA } \\
\text { facility }\end{array}$ & No & Unk & Unk & Unk & Unk & Unk & Unk & Unk & Unk & Unk \\
\hline Oentrich & $200 \mathrm{ft}$ south of CCC/USDA facility & No & 6 & Unk & 1342.47 & 1336.93 & Unk & Unk & Unk & $\sim 150$ & Unk \\
\hline Perkins & 3/4 mi south of CCC/USDA facility & No & Unk & Unk & Unk & Unk & Unk & Unk & Unk & $\sim 60$ & Unk \\
\hline Rothlisberger & $3 / 8$ mi southeast of $C C C / U S D A$ facility & No & Unk & Unk & Unk & Unk & Unk & Unk & Unk & $\sim 100$ & Unk \\
\hline Finch & Approximately $1 \mathrm{mi}$ south of Barnes & No & Unk & Unk & Unk & Unk & Unk & Unk & Unk & Unk & Unk \\
\hline Sedivy & East St. and Railroad Ave. & No & 4 & Unk & Unk & Unk & Unk & Unk & Unk & 138 & Unk \\
\hline \multicolumn{12}{|c|}{ Monitoring wells installed for carbon tetrachloride investigation (construction details reported by BE\&K [1999]) } \\
\hline MW-1S & North of former agricultural bldg & No & 2 & PVC & 1352.60 & 1352.16 & 10 & $13.3-23.3$ & Unk & 23.66 & $12 / 98$ \\
\hline MW-1D & North of former agricultural bldg & No & 2 & PVC & 1352.60 & 1351.92 & 15 & 139.85-159.40 & Unk & 160.53 & $12 / 98$ \\
\hline$M W-2 D$ & Approximately $140 \mathrm{ft}$ north of PWS2 & No & 2 & PVC & 1349.78 & 1349.51 & 15 & $133.26-152.93$ & $128.9-153.37$ & 153.37 & $12 / 98$ \\
\hline MW-3D & $\begin{array}{l}\text { Northeast of intersection of alley and } \\
2^{\text {nd }} \text { Ave. }\end{array}$ & No & 2 & PVC & 1347.03 & 1346.60 & 15 & $133.02-152.73$ & 129-153.33 & 153.33 & $12 / 98$ \\
\hline MW-4D & Former CCC/USDA facility & No & 2 & PVC & 1327.67 & 1326.93 & 15 & $98.38-118.22$ & $94-118.82$ & 118.82 & $12 / 98$ \\
\hline \multicolumn{12}{|c|}{ Monitoring wells installed for Westside Service underground storage tank investigation (construction information from WWC registrations in state database and AEI [2000]) } \\
\hline MW-1 & West of Co-op, south of railroad & Yes & 2 & PVC & 1338.20 & 1337.80 & 35 & 10-45 & 9-45 & 45 & 9/99 \\
\hline MW-2 & West of Co-op, south of railroad & Yes & 2 & PVC & 1337.67 & 1337.19 & 35 & $10-45$ & $9-45$ & 45 & $9 / 99$ \\
\hline MW-3 & West of Co-op, south of railroad & Yes & 2 & PVC & 1337.10 & 1336.81 & 30 & $10-40$ & $9-40$ & 40 & $9 / 99$ \\
\hline MW-4 & West of Co-op, south of railroad & Yes & 2 & PVC & 1337.62 & 1337.29 & 35 & $10-45$ & $9-45$ & 45 & $9 / 99$ \\
\hline MW-5 & West of Co-op, south of railroad & Yes & 2 & PVC & 1336.52 & 1336.17 & 40 & $10-50$ & 9-50 & 50 & $9 / 99$ \\
\hline$M W-7^{a}$ & West of Co-op, south of railroad & Yes & 2 & PVC & 1338.13 & 1337.82 & 40 & $24.5-64.5$ & $23.5-64.5$ & 64.5 & 9/99 \\
\hline$M W-8^{a}$ & West of Co-op, south of railroad & Yes & 2 & PVC & 1337.66 & 1337.29 & 40 & $23.5-63.5$ & $22.5-63.5$ & 63.5 & 9/99 \\
\hline MW-9 & West of Co-op, south of railroad & Yes & 2 & PVC & 1336.09 & 1335.75 & 40 & 40-80 & 39-80 & 85 & 9/99 \\
\hline \multicolumn{12}{|c|}{ Monitoring wells installed during the 2007 investigation by CCC/USDA } \\
\hline MW5 & $175 \mathrm{ft}$ north of Second Ave. & Yes & 2 & PVC & 1327.66 & 1327.20 & 10 & $110-120$ & $108-127$ & 127 & $8 / 2 / 06$ \\
\hline MW6S & $80 \mathrm{ft}$ east of Second Ave. and East St. & Yes & 2 & PVC & 1323.88 & 1323.13 & 10 & $90.5-100.5$ & 88.5-101.5 & 101.5 & $3 / 5 / 07$ \\
\hline MW6D & $80 \mathrm{ft}$ east of Second Ave. and East St. & Yes & 2 & PVC & 1323.88 & 1323.15 & 10 & 105-115 & $104.5-125$ & 125 & $3 / 5 / 07$ \\
\hline MW7 & $60 \mathrm{ft}$ north of Second Ave. & Yes & 2 & PVC & 1330.53 & 1329.91 & 10 & $116-126$ & $114-126$ & 126 & $8 / 6 / 06$ \\
\hline
\end{tabular}


TABLE B.2 (Cont.)

\begin{tabular}{|c|c|c|c|c|c|c|c|c|c|c|c|}
\hline \multirow[b]{2}{*}{$\begin{array}{l}\text { Well } \\
\text { Name }\end{array}$} & \multirow[b]{2}{*}{ Well Location } & \multirow[b]{2}{*}{$\begin{array}{l}\text { WWC-5 } \\
\text { Filed }\end{array}$} & \multirow{2}{*}{$\begin{array}{l}\text { Well } \\
\text { Diameter } \\
\text { (in.) }\end{array}$} & \multirow[b]{2}{*}{$\begin{array}{l}\text { Casing } \\
\text { Type }\end{array}$} & \multicolumn{2}{|c|}{ Elevation (ft AMSL) } & \multirow{2}{*}{$\begin{array}{l}\text { Screen } \\
\text { Length } \\
\text { (ft) }\end{array}$} & \multicolumn{3}{|c|}{ Depth (ft BGL) } & \multirow[b]{2}{*}{$\begin{array}{l}\text { Completion } \\
\text { Date }\end{array}$} \\
\hline & & & & & Ground & Casing & & Screen & Filter & Total & \\
\hline \multicolumn{12}{|c|}{ Monitoring wells installed during the 2007 investigation by CCC/USDA (cont.) } \\
\hline MW8 & $35 \mathrm{ft}$ north of Second Ave. & Yes & 2 & PVC & 1330.78 & 1330.06 & 10 & $110-120$ & $108-120$ & 120 & $8 / 5 / 06$ \\
\hline MW9 & $90 \mathrm{ft}$ east of Center Street & Yes & 2 & PVC & 1319.10 & 1321.86 & 10 & $100-110$ & $98-110$ & 110 & $8 / 2 / 06$ \\
\hline MW10S & $120 \mathrm{ft}$ north of Second Ave. & Yes & 2 & PVC & 1331.57 & 1331.33 & 10 & 93-103 & 91-103 & 103 & 8/17/06 \\
\hline MW10D & $120 \mathrm{ft}$ north of Second Ave. & Yes & 2 & PVC & 1331.57 & 1331.33 & 10 & $115-125$ & $113-125$ & 125 & 8/17/06 \\
\hline MW11S & $120 \mathrm{ft}$ east of Main St. & Yes & 1 & PVC & 1336.70 & 1336.58 & 10 & $40-50$ & $37-52$ & 52 & 9/6/06 \\
\hline MW11M & $120 \mathrm{ft}$ east of Main St. & Yes & 2 & PVC & 1336.70 & 1336.51 & 10 & $90-100$ & $88-102$ & 102 & $9 / 6 / 06$ \\
\hline MW11D & $120 \mathrm{ft}$ east of Main St. & Yes & 2 & PVC & 1336.70 & 1336.53 & 10 & $125-135$ & 123-135 & 135 & 9/6/06 \\
\hline MW12S & $80 \mathrm{ft}$ west of Center St. & Yes & 1 & PVC & 1327.99 & 1327.46 & 10 & $43-53$ & 41-55 & 55 & 9/10/06 \\
\hline MW12M & $80 \mathrm{ft}$ west of Center St. & Yes & 2 & PVC & 1327.99 & 1327.46 & 10 & $90-100$ & 88-102 & 102 & 9/10/06 \\
\hline MW12D & $80 \mathrm{ft}$ west of Center St. & Yes & 2 & PVC & 1327.99 & 1327.52 & 10 & $115-125$ & $113-125$ & 125 & 9/8/06 \\
\hline MW13S & $\begin{array}{l}6 \mathrm{ft} \text { east of Main St. and } 200 \mathrm{ft} \text { south of } \\
\text { Third St. }\end{array}$ & Yes & 2 & PVC & 1342.92 & 1342.36 & 10 & $112-122$ & $110-123$ & 123 & $3 / 1 / 07$ \\
\hline MW13D & $\begin{array}{l}6 \mathrm{ft} \text { east of Main St. and } 200 \mathrm{ft} \text { south of } \\
\text { Third St. }\end{array}$ & Yes & 2 & PVC & 1342.92 & 1342.37 & 10 & $127-137$ & 137 & 137 & $3 / 1 / 07$ \\
\hline MW14S & $\begin{array}{l}10 \mathrm{ft} \text { South of Third St. and } 150 \mathrm{ft} \text { west } \\
\text { of Center St. }\end{array}$ & Yes & 2 & PVC & 1333.08 & 1332.69 & 10 & $108-118$ & $106-119$ & 119 & $3 / 3 / 07$ \\
\hline MW14D & $\begin{array}{l}10 \mathrm{ft} \text { South of Third St. and } 150 \mathrm{ft} \text { west } \\
\text { of Center St. }\end{array}$ & Yes & 2 & PVC & 1333.08 & 1332.74 & 10 & $123-133$ & $122-133$ & 133 & $3 / 3 / 07$ \\
\hline MW15S & $\begin{array}{l}165 \mathrm{ft} \text { east of Center St. and } 560 \mathrm{ft} \\
\text { north of Second Ave. }\end{array}$ & Yes & 2 & PVC & 1309.65 & 1309.34 & 10 & $88-98$ & $87-99$ & 99 & $3 / 6 / 07$ \\
\hline MW15D & $\begin{array}{l}165 \mathrm{ft} \text { east of Center St. and } 560 \mathrm{ft} \\
\text { north of Second Ave. }\end{array}$ & Yes & 2 & PVC & 1309.65 & 1309.29 & 10 & $105-115$ & $104-115$ & 115 & $3 / 6 / 07$ \\
\hline MW16S & $\begin{array}{l}210 \mathrm{ft} \text { north of Second Ave. and } 970 \mathrm{ft} \\
\text { east of Center St. }\end{array}$ & Yes & 2 & PVC & 1299.98 & 1299.47 & 10 & $76-86$ & $74-86$ & 86 & $3 / 9 / 07$ \\
\hline MW16D & $\begin{array}{l}200 \mathrm{ft} \text { north of Second Ave. and } 970 \mathrm{ft} \\
\text { east of Center St. }\end{array}$ & Yes & 2 & PVC & 1299.98 & 1299.52 & 10 & $90-100$ & 88-102 & 102 & $3 / 8 / 07$ \\
\hline MW17 & $\begin{array}{l}170 \mathrm{ft} \text { west of Main St. and } 200 \mathrm{ft} \\
\text { south of Third St. }\end{array}$ & Yes & 2 & PVC & 1352.03 & 1351.77 & 10 & $120-130$ & $118-130$ & 130 & $3 / 9 / 07$ \\
\hline
\end{tabular}

a Westside Service monitoring wells MW-7 and MW-8 were plugged on 4/22/04. 
Argonne

Environmental Science Division

Argonne National Laboratory

9700 South Cass Avenue, Bldg. 203

Argonne, IL 60439-4843

www.anl.gov 